\title{
Reference frames for planning reach movement in the parietal and premotor cortices
}

\author{
Dissertation \\ for the award of the degree \\ “Doctor rerum naturalium” \\ of the Georg-August-Universität Göttingen
}

within the doctoral program Sensory and Motor Neuroscience

of the Georg-August University School of Science (GAUSS)

submitted by

Bahareh Taghizadeh

from Iran

Göttingen 2014 


\section{Thesis Committee}

Prof. Dr. Alexander Gail

Sensorimotor Group, German Primate Center

Prof. Dr. Hansjörg Scherberger

Neurobiology Laboratory, German Primate Center

Prof. Dr. Florentin Wörgötter

III. Physikalisches Institut, Biophysik, Georg-August-Universität

Prof. Dr. Stefan Treue

Cognitive Neuroscience Laboratory, German Primate Center

\section{Memebers of the examination board}

Referee: Prof. Dr. Alexander Gail

Sensorimotor Group, German Primate Center

$2^{\text {nd }}$ Referee: Prof. Dr. Hansjörg Scherberger

Neurobiology Laboratory, German Primate Center

\section{Further members of the examination board}

Dr. Igor Kagan

Decision and awareness Group, German Primate Center

Prof. Dr. Melanie Wilke

Kognitive Neurologie, Universitätsklinikum Göttingen

Date of the oral examination: 17.02.2015 
I hereby declare that this thesis has been written independently and with no other sources and aids than quoted.

Bahareh Taghizadeh

Göttingen 2014 


\section{Acknowledgement}

Here I would like to thank all the people without whom this dissertation would not have been possible.

I would like to first thank my thesis advisor Alexander Gail, a great scientist who patiently supported me through all the steps and gave me the opportunity to learn. I also want to thank my thesis committee members Stefan Treue, Hans Scherbereger and Florentin Woergoetter for their helpful feedbacks and suggestions, and, Igor Kagan and Melanie Wilke who kindly agreed to evaluate my work.

Many people helped me during the time that I was doing my $\mathrm{PhD}$ and had great contribution to the present work. A very special thanks to Sina Plümer and Klaus Heisig, whom without their support handling of the technical issues during the project would have not been possible. I am grateful to Leonore Burchardt and Dirk Prüße for their guidance and helping me with the animal handling, Ralf Brockhausen and Kevin Windolf for handling IT and software problems, and Beatrix Glaser for handling administrative issues.

Science is collaborative in nature, and I have been lucky to have the best colleagues. I would like to thank all the present and former member of the sensorimotor group for supporting me with their feedbacks, suggestions and friendship. I am especially thankful to Stephanie Westendorff from whom I learned about the experimental science, and Pablo Martinez-Vazquez who always supported with his consult and suggestions regarding theoretical issues in my work. I am also thankful to Diemut Regel, Katharina Woergoetter, Tuba Akguel and Diego Giraldo who helped me with collecting psychophysical data.

To my colleagues in the cognitive neuroscience lab, and, decision and awareness group: thank you for making the lab a very friendly atmosphere and thank you for all your scientific feedbacks and suggestions regarding my project.

Last but not least I would like to thank my family, my parents and my sister. Thank you for, believing in me, always encouraging me and supporting me with your love. 


\section{Contents}

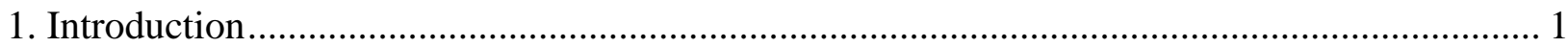

1.1. Two anatomical neural pathways for processing visual information ............................... 2

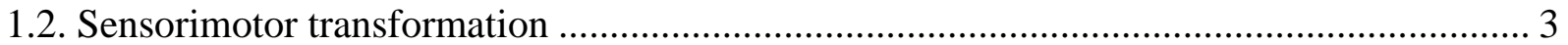

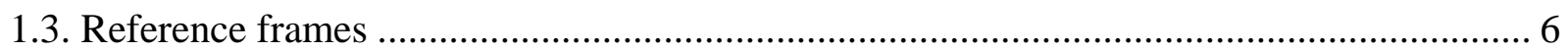

1.4. Flexible behavior and underlying sensorimotor transformation ................................... 8

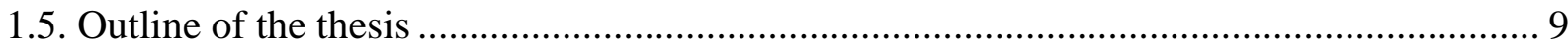

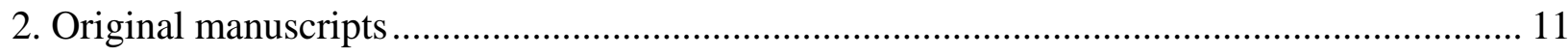

2.1. Spatial task context makes short-latency reaches prone to induced Roelofs illusion........ 12

2.2. Dynamic and scalable object-based spatial selectivity in monkey parietal reach region and

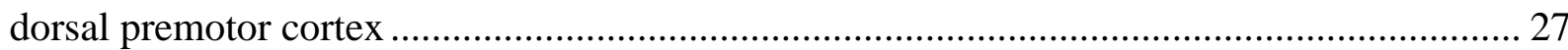

2.3. Asymmetric generalization in adaptation to target displacement errors in humans and in a

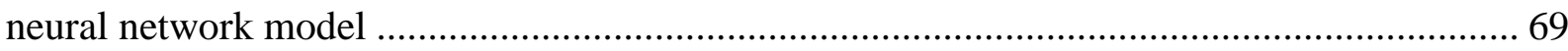

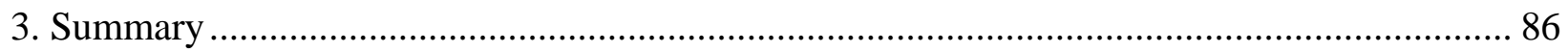

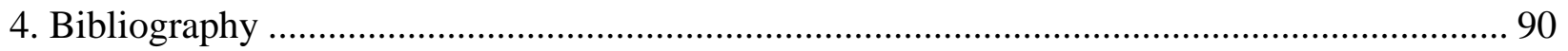

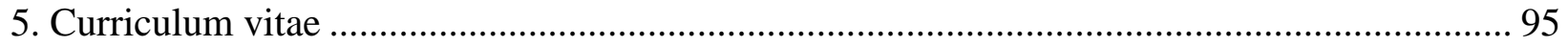




\section{Introduction}

Human and nonhuman primates are able to plan and execute goal-directed behavior and flexibly respond to their surrounding stimuli. Their ability to adapt to changes in their environment is a crucial factor for their survival. The hand of a primate is one its significant features; an important organ which allows physical interaction with objects. Primates use their hands to reach to objects, grasp and manipulate them. Planning a goal-directed hand reaching movement towards an object is not as simple and trivial as it might appear. It is a distributed task that engages many cortical and subcortical areas in primate's brain, prior and during the reaching movement.

The following work addresses planning goal-directed reach movements in human and nonhuman primates. More specifically, the present thesis investigates different representations of spatial parameters which are required for planning a reach movement towards an object. Two complementary approaches were used to study different aspects of this subject. First, psychophysical methods were used to measure and evaluate human behavior in two different behavioral contexts: object-based reach planning where the reach goal needs to be identified relative to an object, and reach goal adaptation task. Second, the underlying neural process was studied in an animal model, a rhesus monkey. The monkey was trained to perform an objectbased reach behavioral paradigm. Activities of single cells in two brain areas which are involved in planning reach movements were measured when the monkey was performing the task under controlled conditions.

The thesis is organized in three chapters. The first chapter is an opening to the topic. It provides a general introduction to sensorimotor transformations and encoding of spatial information during planning of reach movements. The second chapter includes three scientific articles: the first and the third (already published) manuscripts are based on behavioral data from human subjects. The second manuscript (in preparation) is based on the electrophysiology data from monkey. At the beginning of each article a brief introduction is provided, followed by the original manuscript. The third chapter summarizes the results of the presented projects and draws a general conclusion. 


\subsection{Two anatomical neural pathways for processing visual information}

Sensory information provides an internal representation of the outside world to be utilized for performing different behaviors. In primates, vision plays major role in providing contribution to such representation. According to the dual visual system model (Goodale and Milner, 1992; Milner and Goodale, 2008; Mishkin and Ungerleider, 1982) visual information is processed differently for perceptual and motor behavior. Starting from striate cortex, two diverging anatomical pathways were identified (see Figure 1a): the dorsal processing pathway continues through parietal cortex; the ventral processing pathway continues through temporal cortex.

(a)

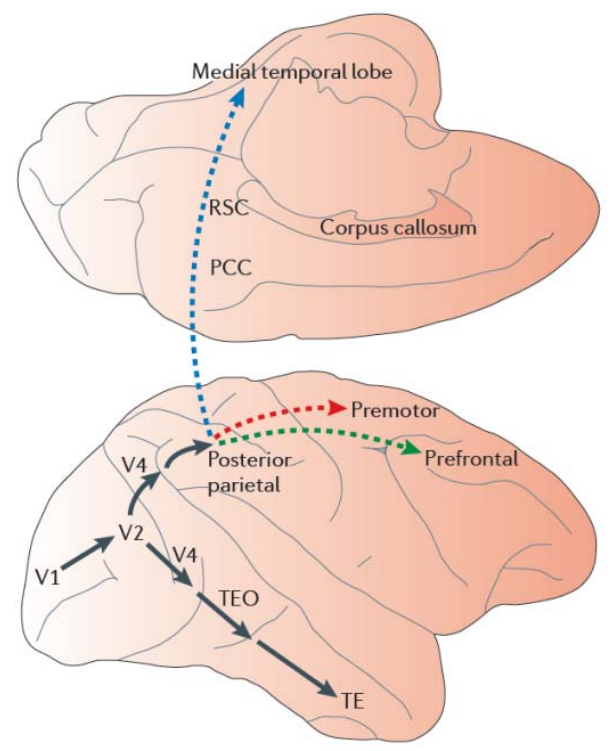

(b)

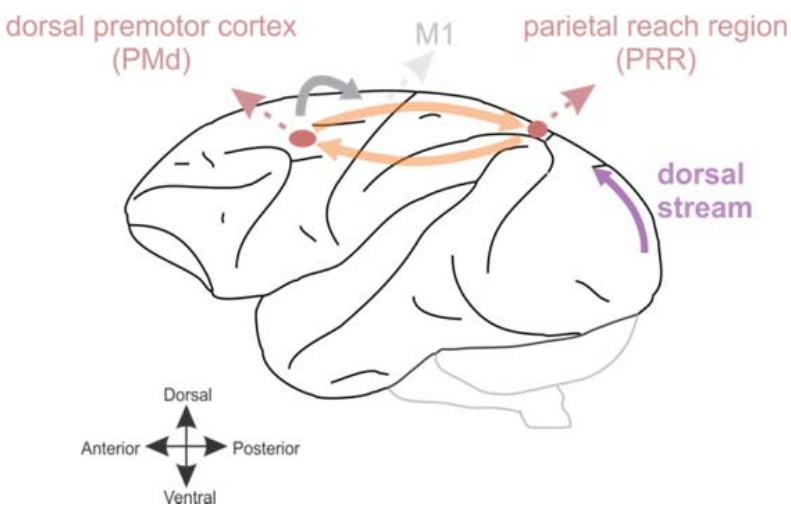

Figure 1: Neural pathways for processing visual information and planning reach movement, (a) two anatomical pathways for processing visual information. The dorsal stream starts from the striate cortex and continues through the parietal cortex; the ventral stream starts from the striate cortex and continues through the temporal cortex. Figure was adapted from (Kravitz et al., 2011). (b) Schematic drawing of reach planning network. The network includes Areas PRR and PMd. Area PMd is projected to the hand area of M1. 
Although the dorsal and ventral streams are tightly interconnected, lesions of brain areas within different streams produce distinct behavioral deficits. Patients with damage in the dorsal stream show spatial disorders: they cannot use visuospatial information to localize objects for reaching towards them, optic ataxia (Andersen et al., 2014); or they are unable to make a precise voluntary arm movement, apraxia (Gross and Grossman, 2008); or they are able to describe single objects but they are unable to perceive more than one object at a time. These patients cannot identify and describe objects using the context information provided by other objects and visual cues in the scene, simultanagnosia (Coslett and Lie, 2008). On the other hand, patients with damage in the ventral stream show deficits in object recognition, visual agnosia (Biran and Coslett, 2003). The strongest neuropsychological evidence (Goodale and Milner, 1992; Milner et al., 1991) was observed in patient D.F. with bilateral damage to the ventral stream. D.F. failed in visual perceptual tasks while her visoumotor performance was not impaired (Milner et al., 1991). Based on such evidence, it was suggested that the two pathways are functionally different. The dorsal stream is believed to be involved in localizing objects as well as planning and controlling actions, whereas the ventral stream is believed to serve perceptual behavior like object identification. This model was also supported by behavioral and psychophysical evidence (see (Westwood and Goodale, 2011) for a recent review).

Although evidence points to the role of the dorsal stream in spatial processing, the nature of processing in this stream is less clear. Dorsal stream was initially proposed to be mediating spatial perception (Mishkin and Ungerleider, 1982) and later was suggested to serve visually guided actions (Goodale and Milner, 1992; Milner and Goodale, 2008). The present thesis is concerned with processing and encoding of spatial parameters in the dorsal stream when planning a goal directed reach movement.

\subsection{Sensorimotor transformation}

Frontoparietal network: In human and nonhuman primates, parietal cortex is located between visual and frontal cortices within the dorsal stream. This portion of the cortex receives inputs from multiple sensory modalities—e.g., (Marconi et al., 2001; Colby et al., 1988)—and is central for integrating sensory and motor systems (sensorimotor transformation). While the anterior parietal cortex is a sensory area, the somatosensory cortex (Iriki et al., 1996), the posterior region of parietal cortex (PPC) has been shown to be involved in action planning (Buneo et al., 2002; 
Snyder et al., 1997; Snyder et al., 2000). Movement planning refers to the neural process that transforms the sensory inputs to generate a motor command for executing an intended movement. This process occurs before initiation of the movement. The PPC consists of several functional subdivisions which are specialized in sensorimotor transformations, subserving motor actions of different effectors, see (Cohen and Andersen, 2002; Wise et al., 1997) for review. While these areas are tightly interconnected, they project to areas of the frontal cortex. Together with their counterparts in the frontal cortex, subdivisions of the PPC form networks within which different motor actions are planned. For instance, the lateral bank of the intraparietal sulcus (area LIP) is anatomically connected to the rostral bank of the arcuate sulcus (the frontal eye field, FEF). Within this network saccadic eye movements are planned. Likewise, the anterior intraparietal area (AIP) which lies along the anterior portion of the intraparietal sulcus, and area F5 of the frontal cortex form a network which is involved in planning hand grasping movements.

Reach planning network: Hand reaching movements are planned within a network which is comprised of parietal reach region (PRR) and dorsal premotor cortex (PMd; see Figure 1b). PRR was functionally identified as a part of PPC which shows activity related to planning a voluntary reach movement (Snyder et al., 2000). Anatomically, PRR mainly includes medial bank of the intraparietal sulcus (MIP) and likely at least part of area V6a (Batista et al., 1999; Snyder et al., 1997). PRR receives inputs from the visual cortex mainly via area V6 of the occipito-parietal circuit (Kravitz et al., 2011).

The area between primary motor cortex (M1) and prefrontal cortex (PFC) is identified as premotor cortex. Anatomical studies have shown bidirectional projections between PRR and PMd (Matelli et al., 1998; Gamberini et al., 2009; Kravitz et al., 2011; Wise et al., 1997; Kurata, 1991; Johnson et al., 1996; Rushworth et al., 2001). PMd projects to the hand area of M1 (Dum and Strick, 2005).

In a simple memory guided reach task, a visual cue stimulus is presented to the monkey. Off set of the cue is followed by a memory period during which monkey has to memorize the location of the cue. The memory period is followed by a go cue indicating to the monkey to leave the initial fixation point and reach to the memorized location of the cue. Activity of single cells within the areas of the reach network is modulated differently during different phases of a trial. 
PRR cells are categorized into different classes in terms of being responsive to presentation of visual instructional cues, during planning of the upcoming reach movement (the memory period) or both (Hwang and Andersen, 2012). This classification holds true for PMd cells as well. It has been shown in both areas that the response of the cells to the visual instructional cues, at least partly relates to the motor significance of the stimuli (Westendorff et al., 2010; Boussaoud et al., 1995). Electrophysiology studies have shown that during the planning phase of a reach movement, activity of single cells in PRR and PMd depends on the spatial direction of the upcoming reach (Buneo et al., 2002; Weinrich and Wise, 1982; Andersen et al., 1985; Boussaoud and Wise, 1993; Kalaska and Crammond, 1995). Neurons show higher firing rate for reaching towards certain directions (which is referred to as the preferred direction, see Figure 2) and less firing rate for other directions. Different neurons have different preferred directions. This dependency of the firing rate on the upcoming movement (spatial selectivity) is referred to as motor goal tuning.

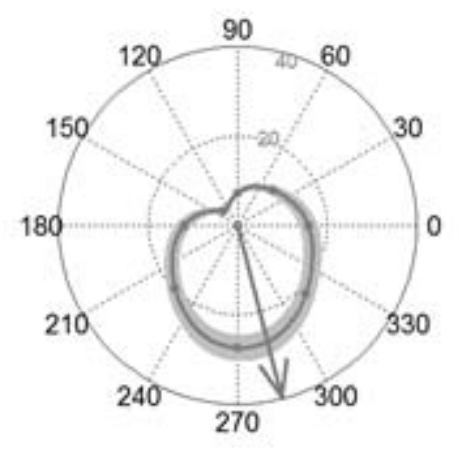

Figure 2: Motor goal tuning. This figure plots mean firing rate of an individual cell when planning reach movement to different directions. In the polar plot, angles represent different movement directions and the amplitude represents mean firing rate of the neuron before reaching to that direction. This cell has higher firing rate when planning a downward reach. This is the preferred direction of this particular cell.

Cells are motor goal tuned when the reaches are directed towards the location of the spatial visual cues. Cells in these areas are also tuned to the reach direction, when the reach goals are inferred from the location of the cues by applying spatial transformations, e.g., pro-anti reach task (Gail and Andersen, 2006; Klaes et al., 2011; Westendorff et al., 2010). Evidence suggests that in the reach planning network, PMd plays a major role in integrating contextual and spatial information during sensorimotor transformation (Cisek and Kalaska, 2010; Westendorff et al., 2010). 


\subsection{Reference frames}

When interacting with the surrounding physical environment and objects, it is a fundamental requirement to have a precise and stable representation of the space available to the brain areas which are preparing the movements. Depending on the behavioral context, different sensory inputs are integrated to plan a reach movement. Various sensory modalities encode the input signals in their specific reference frames. A reference frame refers to a coordinate system within which measured quantities or properties are specified. One of the main challenging issues that arise in sensorimotor transformation is how the brain integrates input signals from different sensory modalities and transforms them into motor commands. According to a traditional view, inputs from different sensory modalities were all transformed into a unified representation in a common coordinate system (Buneo and Andersen, 2006; Cohen and Andersen, 2002; Lacquaniti and Caminiti, 1998; Soechting and Flanders, 1989) which was then used to generate a proper motor command. Alternatively, it has been argued that the brain uses multiple representations of the space to prepare actions, see (Cohen and Andersen, 2002; Battaglia-Mayer et al., 2003) for review. More recent studies have provided evidence that reference frames start from topographic representations in the early stages of sensory processing, e.g., (Medina et al., 2014; Essen and Zeki, 1978) in the sensory cortices and variety of reference frames are developed in higher cortical areas, for example, (Batista et al., 2007; Bernier and Grafton, 2010; Beurze et al., 2010; Chafee et al., 2007; Colby, 1998; Committeri et al., 2004; Galati et al., 2010; McGuire and Sabes, 2009) which are then employed in sensorimotor transformation. For instance neurons in primary visual cortex (V1) encode the location of visual stimuli in a coordinate system which is anchored to retina, in other words in the retinotopic coordinate frame (Essen and Zeki, 1978). To plan a visually guided reach movement, visual inputs need to be transformed into motor commands which are encoded in joint-centered coordinates to move the hand (Caminiti et al., 1991; Crawford et al., 2004; Kakei et al., 1999; Kalaska et al., 1997; Scott and Kalaska, 1997; Scott and Kalaska, 1995).

Spatial frames of reference: In spatial cognition and memory research, putative coordinate systems which could be used for spatial encodings are divided into two broad categories (see Figure 3): egocentric reference frames in which locations are encoded relative to the observer or a part of its body; allocentric reference frames in which locations are encoded relative to 
environmental landmarks or relative to the present objects in the environment, independently of the observer's position. Allocentric coordinate systems could be anchored either to the environmental landmarks or on the objects in the peripheral space, within a reach distance. The former provides reference frames to form a spatial map of a wide spread space which then could be exploited for navigation through the space; the latter provides a reference frame (that is an object-centered reference frame) which could be used for interaction with objects that are located within the reaching distance. The topic of interest in the present thesis is the object-centered reference frame; therefore the rest of the thesis is focused on this form of allocentric representation.

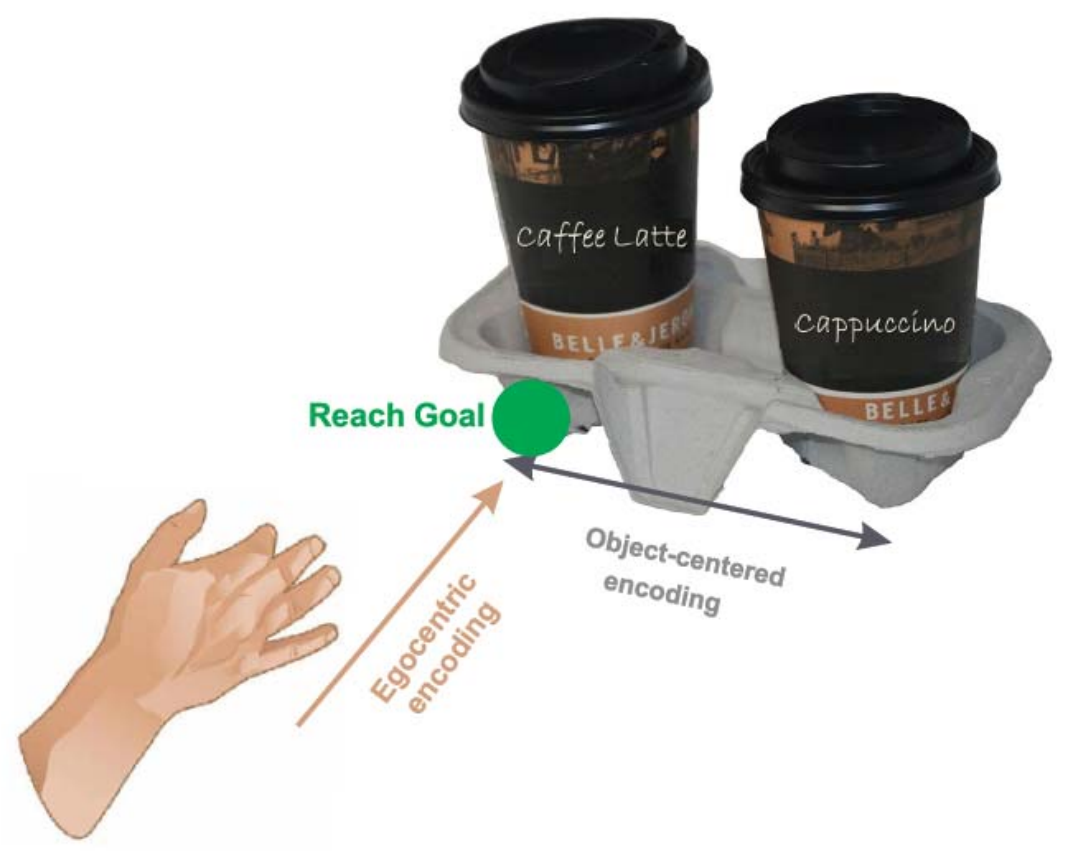

Figure 3: Directing reach towards an object. When reaching towards a coffee tray (the object) to take one of the two cups of coffee, reach goal has to be identified relative to the object, in object-centered reference frame. In this example subject is reaching to the left end of the object. The reach goal also has to be encoded relative to the subject's body, in egocentric reference frame. (Illustration: http://thepapercupcompany.trustpass.alibaba.com/)

Object-centered reach planning: When reaching towards objects, in certain behavioral contexts the reach goal needs to be initially identified relative to the object towards which the reach movement is directed. The target of the reach could be a specific part of the object, for instance middle or one corner of the object. Thus the reach goal initially needs to be identified within the 
object, in the object-centered reference frame. Imagine a paper coffee tray holding two coffee cups and you would like to take one of the cups (see Figure 3). Depending on which cup you would like to take, you should direct your reach to the left end or right end of the tray. The tray is the object towards which you reach. Therefore your reach goal initially has to be defined relative to the paper tray (in object centered reference frame).

Based on the evidence from behavioral and imaging studies in perceptual spatial judgment (Committeri et al., 2004; Galati et al., 2000; Galati et al., 2000; Vallar et al., 1999; Chen et al., 2014; Zaehle et al., 2007) processing of egocentric and object-centered encodings was initially assigned to the dorsal and ventral streams, respectively (Goodale and Westwood, 2004; Milner and Goodale, 2008). Recent imaging studies investigated the neural circuitry that subserves encoding of spatial parameters of the reach when human subjects were performing movements in different behavioral contexts. Results suggest that neural circuits which process the two types of representations are not strictly separated but rather partly overlapped within sub regions of the dorsal stream (Thaler and Goodale, 2011; Chen et al., 2014). However, this issue was never studied at the level of single cells, during planning reach movements. It is not clear whether cells in the reach planning network can encode the motor goal in object-centered as well as egocentric reference frame? It is also not clear whether there is any difference between areas PRR and PMd when encoding object-centered reference frame.

\subsection{Flexible behavior and underlying sensorimotor transformation}

Flexible behavioral responses to the changing environment require adjustable sensorimotor transformation. Depending on the behavioral context adjustment can happen in different time scales: fast and slow. For instance in certain behavioral contexts, at different stages of movement planning, different spatial parameters may be used. Imagine the coffee tray example again. To take a coffee cup from the tray, the reach goal initially has to be identified relative to the tray (that is, in the object-centered reference frame). Later in order to move the hand towards the target, reach goal has to be identified in different egocentric reference frames such as joints and body. In such scenario the adjustment demands a transition between two types of representation (from object-centered to egocentric) within a few hundred milliseconds. Therefore two different modes of transformation of sensory inputs to motor outputs are relevant for behavior at different times during the movement planning. It is interesting to know when the behavioral task demands 
such fast adjustment in sensorimotor transformation, how is this reflected in the activity of individual cells in the reach planning network? Do individual cells also adjust their encoding according to the behavioral relevance of the encoded parameter?

In some other behavioral contexts, adjustment requires learning a new mapping between sensory inputs and motor outputs. This situation usually happens when sensorimmotor transformation leads to errors in different aspects of the movement due to a new environmental condition. In order to have proper sensorimotor transformation, through a learning procedure the underlying neural network is gradually modified to compensate for the environmental changes. Such modification is referred to as sensorimotor adaptation. Prism adaptation, exemplifies such gradual modification in sensorimotor transformation. In prism adaptation, e.g., (Martin et al., 1996), healthy human subjects are asked to wear prism glasses that laterally shift their visual field with a constant amount. Subjects are asked to point to a target point in front of them. The new environmental condition (looking through the prism) induces a misalignment between visual and properioceptive feedbacks of the location of the hand. Therefore, in the first few trials they make large errors in the location towards which they point. The error gradually decreases and after few trials they can precisely point to the target. In the prism example, the entire visual field is affected by the prism. Alternatively, the manipulation could be applied only to a limited part of the space. In that case, it is intriguing to know how does learning a new sensorimotor transformation for manipulated part of the space, affect sensorimotor transformation for unmanipulated parts of the space and how is it reflected in the motor performance of the subject?

\subsection{Outline of the thesis}

In the current thesis an object-based reach behavioral paradigm was designed which allowed us to study two different aspects of object-based reach planning. This paradigm was used for a psychophysics study on healthy human subjects. We investigated how visual contextual stimuli, which were task irrelevant and had to be ignored during reach movement planning, could affect reach performance. We compared visual contextual information effects on reach performance of healthy subjects when they were asked to plan reach movements in egocentric reference frame, with that when they planned reach movement in object-centered reference frame. 
Although behavioral parameters can partly reflect the functional properties of neural circuits which drive the behavior, they do not provide a precise representation of processing at the level of individual cells. To gain a better understanding of how single cells encode different reference frames during reach movement planning, a variation of the object-based reach behavioral paradigm was used for a monkey electrophysiology experiment. We studied how cells in monkey's PRR and PMd encode spatial parameters when the monkey was asked to direct his reach towards a task relevant object. Specifically we studied whether single cells in PRR and PMd encode the reach goal relative to the object (in object-centered reference frame) as well as relative to the monkey's body (in egocentric reference frame). In a third investigation we conducted a psychophysics experiment on human subjects, in the context of motor adaptation. We asked how egocentric reference frames which are used for planning reach movement adjust when the visually instructed reach goal is perturbed. 


\section{Original manuscripts}

This chapter contains the following manuscripts:

1. Taghizadeh B and Gail A (2014) Spatial task context makes short- latency reaches prone to induced Roelofs illusion. Front. Hum. Neurosci. 8:673. doi: 10.3389/fnhum.2014.00673.

2. Taghizadeh B and Gail A, Dynamic and scalable object-based spatial selectivity in monkey parietal reach region and dorsal premotor cortex (in preparation)

3. Westendorff, S, Kuang, S, Taghizadeh, B, Donchin, O, Gail, A, Asymmetric generalization in adaptation to target displacement errors in humans and in a neural network model. Journal of Neurophysiology 113(7): 2360-2375. doi: 10.1152/jn.00483.2014

\section{Author's contributions:}

1. B.T. and A.G. designed the experiment, B.T. collected the data, did the analysis and wrote the manuscript. A.G. edited the manuscript. All authors discussed the results and commented on the manuscript at all stages.

2. B.T. and A.G. designed the experiment, B.T. collected the data, did the analysis and wrote the manuscript. A.G. edited the manuscript. All authors discussed the results and commented on the manuscript at all stages.

3. S.W. and A.G. designed the experiment. S.W. and B.T. collected the data. S.K., S.W. and A.G. designed the model. S.K. and S.W. ran the model simulation. S.W. did the analysis and wrote the manuscript. A.G., S.W. and O.D. edited the manuscript 


\subsection{Spatial task context makes short-latency reaches prone to induced Roelofs illusion}

Visual contextual information normally helps to better identify and localize objects. However, visual contextual stimuli some time have erroneous influence on processing of visual information, which is known as visual illusion. It is a common belief that processing of visual information in the ventral stream to subserve perception is prone to visual illusions, whereas processing of visual information in the dorsal stream to subserve motor actions is robust to illusions. In this study we investigated how contextual stimuli influence reach performance of healthy human subjects.

In order to study effect of task-irrelevant contextual cues, we designed a variation of the induced Roelofs illusion behavioral paradigm. In the induced Roelofs effect, position of a task irrelevant visual object (contextual cue) induces a systematic shift in the localization of the visual target object. We compared the effect of the contextual cue on the reach performance of subjects when short-latency reaches were planned in object-centered reference frame with that when shortlatency reaches were planned in egocentric reference frame. We found that during objectcentered encoding of the motor goal locations, the information of additional task-irrelevant object can induce systematic mis-localizations of the reach goal. This was not the case for egocentric encoding of the motor goal. Results suggest that during sensorimotor transformation, the illusory influence of visual contextual cues is transformed in specific reference frames, here in object-centered reference frame. 


\title{
Spatial task context makes short-latency reaches prone to induced Roelofs illusion
}

\author{
Bahareh Taghizadeh ${ }^{1,2}$ and Alexander Gail 1,2,3 * \\ ' Sensorimotor Group, German Primate Center, Leibniz Institute for Primate Research, Göttingen, Germany \\ ${ }^{2}$ Faculty of Biology and Psychology, Georg-August-Universität, Göttingen, Germany \\ ${ }^{3}$ Bernstein Center for Computational Neuroscience, Göttingen, Germany
}

Edited by:

J. Douglas Crawford, York

University, Canada

\section{Reviewed by:}

Lore Thaler, Durham University, UK

Patrick A. Byrne, University of

Toronto, Canada

*Correspondence:

Alexander Gail, Sensorimotor Group, German Primate Center, Leibniz Institute for Primate

Research, Kellnerweg 4, Göttingen 37077, Germany

e-mail: agail@gwdg.de
The perceptual localization of an object is often more prone to illusions than an immediate visuomotor action towards that object. The induced Roelofs effect (IRE) probes the illusory influence of task-irrelevant visual contextual stimuli on the processing of task-relevant visuospatial instructions during movement preparation. In the IRE, the position of a taskirrelevant visual object induces a shift in the localization of a visual target when subjects indicate the position of the target by verbal response, key-presses or delayed pointing to the target ("perception" tasks), but not when immediately pointing or reaching towards it without instructed delay ("action" tasks). This discrepancy was taken as evidence for the dual-visual-stream or perception-action hypothesis, but was later explained by a phasic distortion of the egocentric spatial reference frame which is centered on subjective straight-ahead (SSA) and used for reach planning. Both explanations critically depend on delayed movements to explain the IRE for action tasks. Here we ask: first, if the IRE can be observed for short-latency reaches; second, if the IRE in fact depends on a distorted egocentric frame of reference. Human subjects were tested in new versions of the IRE task in which the reach goal had to be localized with respect to another object, i.e., in an allocentric reference frame. First, we found an IRE even for immediate reaches in our allocentric task, but not for an otherwise similar egocentric control task. Second, the IRE depended on the position of the task-irrelevant frame relative to the reference object, not relative to SSA. We conclude that the IRE for reaching does not mandatorily depend on prolonged response delays, nor does it depend on motor planning in an egocentric reference frame. Instead, allocentric encoding of a movement goal is sufficient to make immediate reaches susceptible to IRE, underlining the context dependence of visuomotor illusions.

Keywords: reach movement, induced Roelofs effect, illusion, reference frame, allocentric, object-centered

\section{INTRODUCTION}

Goal-directed, object-oriented reach movements require accurate localization of the target object, yet object localization can be prone to visual illusions. The fact that in many cases visual perceptual localization is more prone to illusions than immediate visuomotor responses (Smeets and Brenner, 2001) is typically taken as strong evidence for two functionally independent visual processing streams, a ventral "vision-for-perception" pathway, and a dorsal "vision-for-action" pathway (Goodale and Milner, 1992; see Schenk et al., 2011 and Westwood and Goodale, 2011 for recent reviews). Understanding the circumstances under which perceptual illusions do or do not affect motor performance can be highly informative about the nature of the two putative visual streams, and, more specifically, about the nature of visuospatial processing during sensorimotor transformations (Smeets et al., 2002). Here we re-investigate the induced Roelofs effect (IRE) in reach movements. In the IRE, the position of a task-irrelevant visual object induces a shift in localization of the target object.
The IRE depends on the mode of the subjects' behavioral response to indicate this position, e.g., key-presses vs. immediate reaches towards the target (see details below). This observation was originally taken as evidence for the dual-visual-stream or perceptionaction hypothesis (Bridgeman et al., 1997), attributing the IRE to ventral stream perceptual processing. A later, opposing view explained the IRE by a phasically distorted egocentric (object-toself) reference frame-i.e., changes in space defined relative to the own body-attributing the IRE to dorsal stream processing along the vision-to-action pathway (Dassonville and Bala, 2004b). Here we expand on these findings by revisiting the IRE in a short-latency reach task. In the first experiment, different to previous studies, we varied the spatial task context in which reaches had to be performed. We distinguished reaches in an allocentric (object-to-object) reference frame, i.e., a task in which the reach goal location is defined relative to another object, from otherwise identical reaches in an egocentric reference frame, i.e., reach goals relative to the own body. We thereby test if the 
IRE can also be induced for immediate reaches to the target (typically considered an "action" task) if the spatial context of the task is modified. In a second experiment, we test if the IRE critically depends on a phasic distortion of an egocentric frame of reference or if it can also be induced by allocentric encoding.

The IRE probes the illusory influence of task-irrelevant visual context stimuli on the processing of task-relevant visuospatial instructions during movement preparation. Note that taskrelevance here refers to whether a stimulus was instructive for subjects, independent of its effect on behavior. In a series of studies Bridgeman et al. (1997, 2000) showed that the position of a task-irrelevant visual object (frame) can induce a systematic shift in localization of visual targets. When the frame was laterally off-center relative to subjects' mid-sagittal plane, i.e., the frame was shifted to the left or right with respect to the subjects' body midline, subjects misjudged the position of targets presented inside the rectangular frame (Bridgeman et al., 1997). The mislocalization was in the opposite direction of the frame shift, i.e., if the frame was left of the midline then targets were mislocalized to the right, and vice versa. Target mislocalization occurred in two conditions. First, when subjects had to indicate target position by pressing response keys assigned to different targets. The keyboard was placed on the table in front of the subjects, and hence the keys were spatially incongruent to the actual target positions. Second, when subjects pointed to the memorized position of the target after an instructed delay (Bridgeman et al., 1997). Importantly, when subjects in the same task indicated the target position without instructed delay by either pointing to it (Bridgeman et al., 1997) or by directly reaching to jab at the target (Bridgeman et al., 2000), no IRE was observed. This discrepancy was originally interpreted as an indication of separate visuospatial representations for direct sensorimotor processing (immediate reaching or pointing without instructed delay) in the dorsal visual stream, compared to spatial cognitive or perceptual processing (verbal response, using response keys, or pointing with instructed delay) in the ventral visual stream. This dual-visualstream or perception-action hypothesis of the IRE was based on two assumptions. First, only the perceptual "cognitive" ventral stream is prone to the IRE illusion. Second, only the immediate and directly target-aimed manual responses can be performed by direct egocentric sensorimotor processing in the dorsal stream. Symbolic responses (verbal response or pressing of response keys) and delayed memory-guided reaching and pointing, on the other hand, require ventral stream processing (Bridgeman et al., 1997, 2000). In case of visually instructed delayed reaching and pointing, the need for ventral stream processing arises from the assumption that the dorsal vision-to-action pathway is incapable of even medium-term (several seconds) memory storage of the required reach parameters, while immediate reaches can be processed by the dorsal stream alone, as further discussed below.

Behavioral and imaging studies challenged this interpretation of the IRE in favor of an alternative biased-midline hypothesis (Dassonville and Bala, 2004b; Dassonville et al., 2004) in which the IRE is explained by a temporary distortion of the egocentric spatial frame of reference which is used for reach planning and which is centered on the direction of the subjective straight-ahead (SSA; see Figure 1). Dassonville and colleagues showed that the IRE can be accounted for by an observed mislocalization of the memorized array of reference positions, relative to which the target position had to be indicated with a saccade. Since the mislocalization of the memorized reference positions occurred in the same direction as the off-centered visual frame it explained the observed target localization error opposite to the off-centered frame. This finding was interpreted as indication of a phasic translational shift in an egocentric reference frame which is used for movement planning, and which is centered on the direction of SSA (Dassonville and

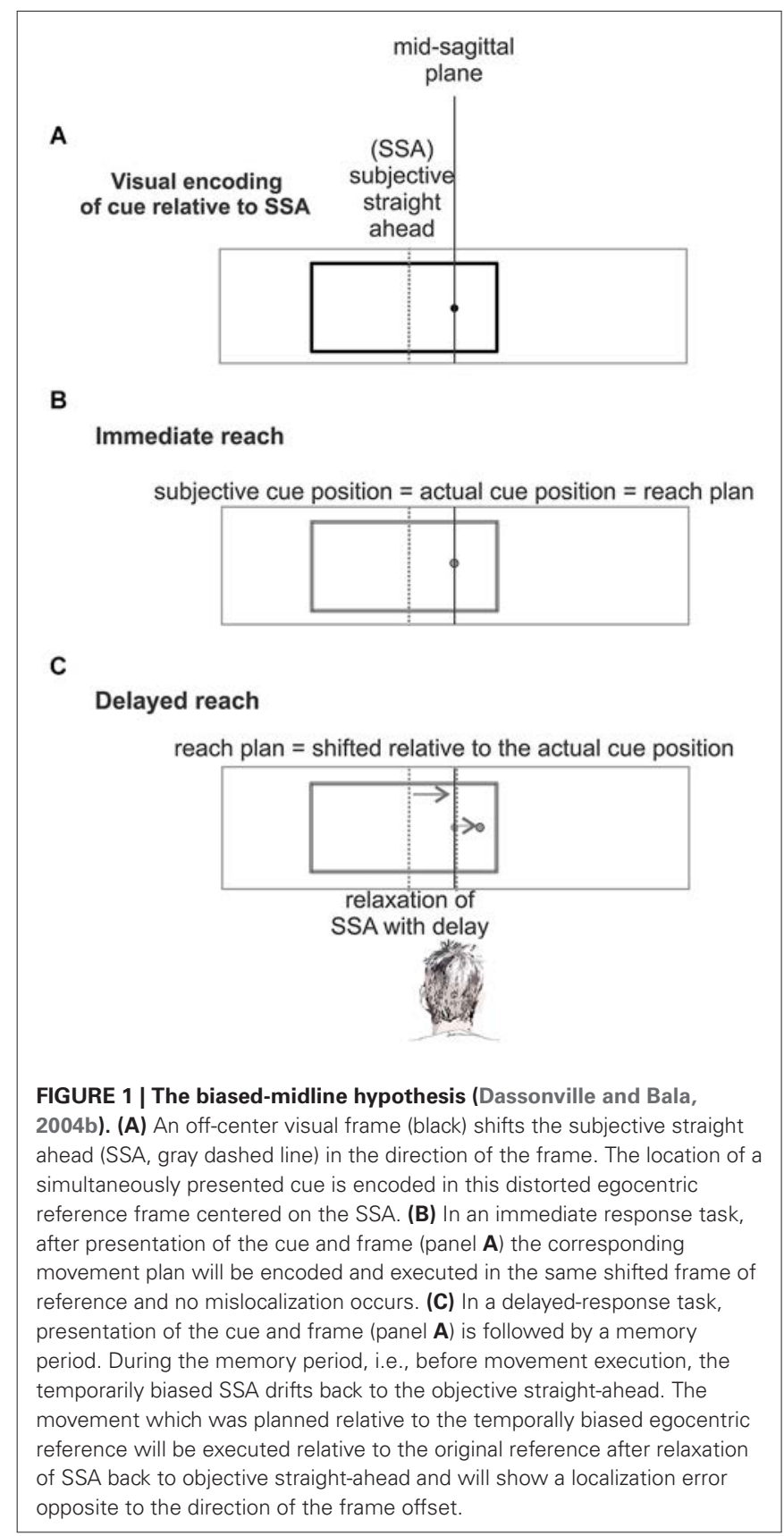


Bala, 2004b; Dassonville et al., 2004). According to this biasedmidline hypothesis, in an immediate motor response task (nondelayed pointing, reaching, or saccade) the target location and the corresponding movement plan will both be encoded in the phasically shifted egocentric frame of reference, and the movement plan will be executed while the reference frame is still shifted. No obvious movement error occurs, since movement planning and execution are both affected by the shift, and hence the shift is compensated (Figure 1B). In a delayed-response task, the movement will be executed after relaxation of the shifted SSA back to the mid-sagittal plane. This induces a target error to the direction opposite to the off-set visual frame, since the movement was planned relative to the SSA but executed relative to the original un-biased frame of reference after relaxation (Figure 1C).

An fMRI study of the IRE revealed differential activity in the dorsal visual stream but not in the ventral stream (Walter and Dassonville, 2008). The dual-visual-stream hypothesis would have pointed to a main contribution from the ventral stream for IRE-prone behavioral conditions. In contrast, the biasedmidline hypothesis implies that the IRE is based on a single egocentric visuospatial reference frame, likely in the dorsal visual stream, which would be relevant for both the IRE-resistant "sensorimotor" or "action" tasks (immediate target-directed manual or ocular response) and the IRE-prone "cognitive" or "perceptual" versions of the task (delayed pointing and looking or symbolic responses). However, the localization of IRErelated neural activity in the dorsal stream does not answer the questions of which spatial reference frame and which temporal dynamics determine the behavioral consequences of the IRE. The previously suggested dual-visual-stream model for the IRE is tied to the perception-action model (Goodale and Westwood, 2004; Goodale et al., 2004), according to which the ventral and dorsal visual streams are preferentially associated with allocentric and egocentric processing, respectively. On the other hand, there is growing evidence for parallel existence of both spatial reference frames within the dorsal visual pathway (Burgess, 2006; Milner and Goodale, 2008) and it is clear that the brain uses both types of information for localization of spatial targets in many tasks (Byrne and Crawford, 2010). Accordingly, spatial locations are not purely encoded in egocentric frames of reference in the posterior parietal cortex. The fMRI-active areas in the Dassonville IRE study (Walter and Dassonville, 2008) overlapped not only with areas shown in previous experiments to be involved in egocentric spatial localization (Vallar et al., 1999), but also with areas involved in allocentric localization relative to immediate visual objects (Galati et al., 2000; Thaler and Goodale, 2011a) or the enduring spatial features of a familiar environment (Galati et al., 2010). In addition, Fink et al. (2003) showed that egocentric and allocentric (object-centered) reference frames can interact in the human parieto-frontal network. Although there are not many studies directly comparing egocentric and allocentric reference frame in monkeys, there is evidence that neurons in parietal area 7 a can encode the spatial location of objects in an eye-centered (i.e., egocentric) reference frame (Andersen et al., 1985) as well as relative to other task-relevant objects (Chafee et al., 2007; Crowe et al., 2008). Neurons in the same area are gain-modulated by the position of the subject's body in the surrounding environment (i.e., in world-centered reference frame) (Snyder et al., 1998). The original dual-visual-stream hypothesis for the IRE argued that the dorsal stream, which dominates immediate egocentric "action" tasks, makes use of the ventral stream information only in case of memory-guided tasks. This explains the susceptibility of reaches to the IRE when they are substantially delayed by several seconds (Bridgeman et al., 1997, 2000; Dassonville and Bala, 2004b).

In summary, both existing interpretations of the IRE, namely the dual-visual-stream and the biased-midline hypothesis, critically depend on the following observation: in tasks in which subjects are required to directly point to, look at, or touch the perceived target position, and in which they can do so in an egocentric reference frame, the IRE can be observed if the manual or ocular response is purposefully delayed by several seconds, but not if an immediate response is required (Bridgeman et al., 1997, 2000; Dassonville and Bala, 2004b). Since the biased-midline hypothesis assumes a distortion of an egocentric reference frame (a shifted SSA) which is only phasic, it predicts that immediate reaches should be resistant to IRE because visual encoding of the reference positions and the reach target are affected in the same way. The dual-visual-stream hypothesis, on the other hand, assumes that dorsal stream processing utilizes ventral stream information only in memory-guided action, hence, it predicts resistance to the IRE for immediate reaches in an egocentric reference frame, but makes no prediction about immediate targetaiming reaches in other reference frames. In experiment I we test if immediate reaches, independent of a prolonged reach delay, can become prone to IRE if the task context prevents egocentric reach planning. To dissociate egocentric from allocentric reach planning, we introduced a spatially incongruent object-centered reach task. In contrast to previous IRE reaching experiments, we also introduced ocular fixation constraints. Furthermore, the fact that the dorsal stream areas which are active during target localization in IRE tasks cover areas of egocentric as well as allocentric spatial encoding brings up the second and related question of whether the IRE is really restricted to phasic distortion effects on egocentric frames of reference induced by the relative position of an object to the body. If not, mislocalization effects like the IRE might also be induced by the relative (allocentric) position of an object relative to another object. Previous IRE experiments including allocentric task constraints were nevertheless still explained by egocentric causes (Dassonville and Bala, 2004b; Lester and Dassonville, 2011). In Experiment II we tested whether the IRE can interfere with allocentric reach planning and can thus be explained independently of an egocentric reference frame distortion.

\section{MATERIALS AND METHODS APPARATUS}

Subjects were seated in a dimly lit room in front of a frontoparallel touch screen $(43 \mathrm{~cm}$ distance from eye, screen center at eye level) so that their mid-sagittal plane was aligned to the center of the screen. Visual stimuli were presented on an LCD screen (19" ViewSonic VX922) mounted behind a touch-sensitive screen (IntelliTouch, ELO Systems, CA, USA). Custom-written display software $(\mathrm{C}++)$ was controlled via a real-time program 
running on a PXI computer (LabView, National Instruments). Stimulus display was synchronized with vertical synchronization of the screen to avoid latency jitter. Visual display latencies were recorded with a photo diode and corrected for during data analysis. All visual stimuli had a low intensity gray tone $\left(9.0 \mathrm{~cd} / \mathrm{m}^{2}\right.$ on a $1.2 \mathrm{~cd} / \mathrm{m}^{2}$ background) to minimize retinal afterimages. Hand position was registered using the touch screen. Gaze positions were registered using an infrared eye tracker at $500 \mathrm{~Hz}$ (SMI, Teltow, Germany, in experiment I and EyeLink 1000, Kanata, Canada, in experiment II). Subjects rested their head on a chinrest for stability. Behavioral parameters were monitored using the real-time control software.

\section{SUBJECTS}

All subjects had normal or corrected-to-normal vision and were naïve with regard to the purpose of the study. Detailed written instructions were given to the subjects before each experiment. Experiments were in accordance with institutional guidelines for experiments with humans and adhered to the principles of the Declaration of Helsinki. All subjects gave their informed written consent prior to their inclusion in the study.

Eleven right-handed subjects (20 to 27 years, four females) participated in experiment I and control experiment Ia. Nine different right-handed subjects (22 to 39 years, five female) participated in control experiment Ib. A disjunct group of subjects was necessary for this control task to avoid possible task interference with experiment I. Ten different right-handed subjects (16 to 27 years, five females) participated in experiment II and control experiment IIa.

\section{EXPERIMENTAL PARADIGM}

The following procedures for implementing the IRE were common to both experiments. Details specific for the individual experiments, especially the spatial positioning of stimuli, will be described in experiments I and II below.

Each trial started with an eye-fixation target, presented to the subject at the vertical midline (mid-sagittal plane), and $5 \mathrm{~cm}$ ( $7^{\circ}$ visual angle) above the horizontal midline of the screen (Figure 2A). Subjects were required to fixate the spot throughout each trial within an invisible window of $3 \mathrm{~cm}\left(4^{\circ}\right.$ ) radius (ocular fixation). To start a trial, subjects had to push a "home" button, placed on subject's mid-sagittal plane on the desk $40 \mathrm{~cm}$ below the screen center, and keep it pressed with their index finger until a "go" signal occurred later in the trial (manual fixation). Whenever subjects failed to keep ocular or manual fixation, the trial was aborted and repeated at a random later time during the experiment. After valid eye and hand fixation of 500-700 ms, a reference array (RA) of five boxes, horizontally connected with a line, appeared for $200 \mathrm{~ms}$. Boxes were $0.35 \mathrm{~cm}\left(0.5^{\circ}\right)$ squares, and centered $1.5 \mathrm{~cm}\left(2^{\circ}\right)$ apart from each other. The position of the boxes indicated the potential positions of the pending cue. Subjects were required to keep these positions in mind for proper task performance, as will become clear below. Reference array presentation was followed by a memory period of $3 \mathrm{~s}$. After the memory period a visual cue was presented for $200 \mathrm{~ms}$. The cue consisted of a small dot of $0.27 \mathrm{~cm}\left(0.35^{\circ}\right)$ diameter at the randomly chosen position of one of the five RA boxes, indicating the target box to which subjects should later reach. The cue was surrounded by a simultaneously presented frame, which was $16.9 \mathrm{~cm}\left(21^{\circ}\right)$ wide and $6.6 \mathrm{~cm}\left(9^{\circ}\right)$ high, but which was task-irrelevant. Cue and frame were succeeded by a decision array (DA), which was graphically identical to the RA, but was not necessarily spatially congruent (see below). Stimulus-onset asynchrony (SOA) between "cue + frame" and the subsequent DA was 200-300 ms. Simultaneously to the appearance of the DA, an acoustic signal was presented for 50 $\mathrm{ms}$ as the go-signal, indicating to the subject to lift their finger from the home button and touch the target position on the screen within $1000 \mathrm{~ms}$ after the go signal. After a correctly executed trial, subjects received acoustic feedback (high-pitched tone).

One constraint common to both experiments was that the frame could randomly take one of three possible positions relative to the RA: centered, or shifted by $3.85 \mathrm{~cm}\left(5^{\circ}\right)$ to the left or right. Another constraint common to all experiments was that the cue appeared at one of the five RA positions. Subjects were instructed to hit the one of the five DA boxes which corresponded to the RA box at which they had perceived the cue, e.g., for a cue perceived at RA box \#2 subjects should reach to DA box \#2, irrespective of the absolute position of the DA. If the reach endpoint was within $4.5 \mathrm{~cm}\left(6^{\circ}\right)$ distance from the target box the trial was counted as "successful". By tolerating off-sets up to three boxes distant from the physically cued target box, we could analyze localization errors without inducing behavioral biases from error feedback. In the following sections, for each trial of the task the term "cue" refers to the dot stimulus presented simultaneously with the frame (Figure 2A, spatial cue + frame) and "target" refers to the position of the relevant box of the DA (i.e., the box of the DA that corresponds to the cued box of the RA).

Before entering the experiment, all subjects completed a training session and were encouraged to ask any questions which were not answered by the written instructions. The aim of the behavioral training was to familiarize subjects with object-based (allocentric) reach planning. More details on the training task will be elaborated for each experiment separately in the following sections.

\section{EXPERIMENT I}

The main conclusion of this study will result from Experiment II. But since Experiment II differs in multiple respects from previous implementations of IRE tasks, we first wanted to establish some basic findings in our type of experimental setting to make the data more comparable to previous experiments. In experiment I, we asked what determines the "immediacy" of the reaches which previously did not show an IRE. Is it only the time between the presentation of the cue that instructs the target and the reach onset which determines whether the IRE occurs or not, or can the spatial frame of reference in which the cue and target have to be encoded cause an IRE even when other spatial and temporal reach parameters are matched? Experiment I and control experiment $\mathrm{Ib}$ aim to distinguish between these two alternatives by introducing a task in which reaches can be conducted without instructed delay ("temporally immediate") but might be associated with a spatially non-congruent target 


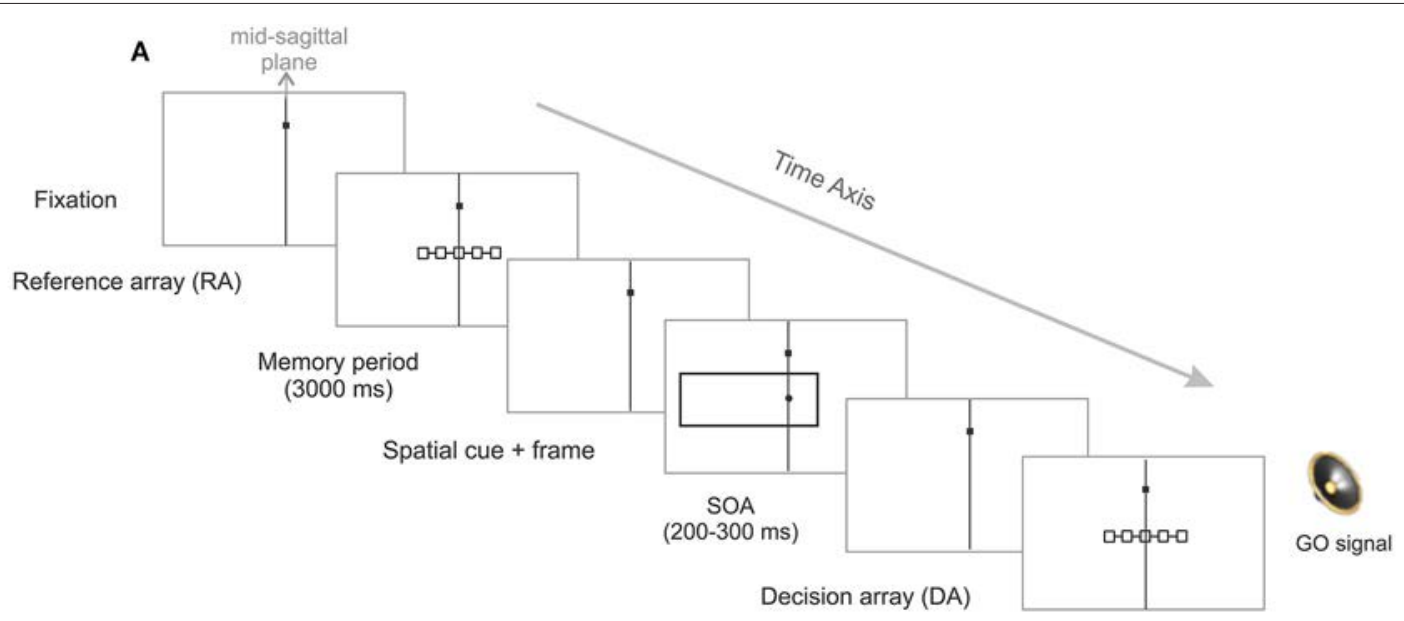

B Experiment I

C Experiment II
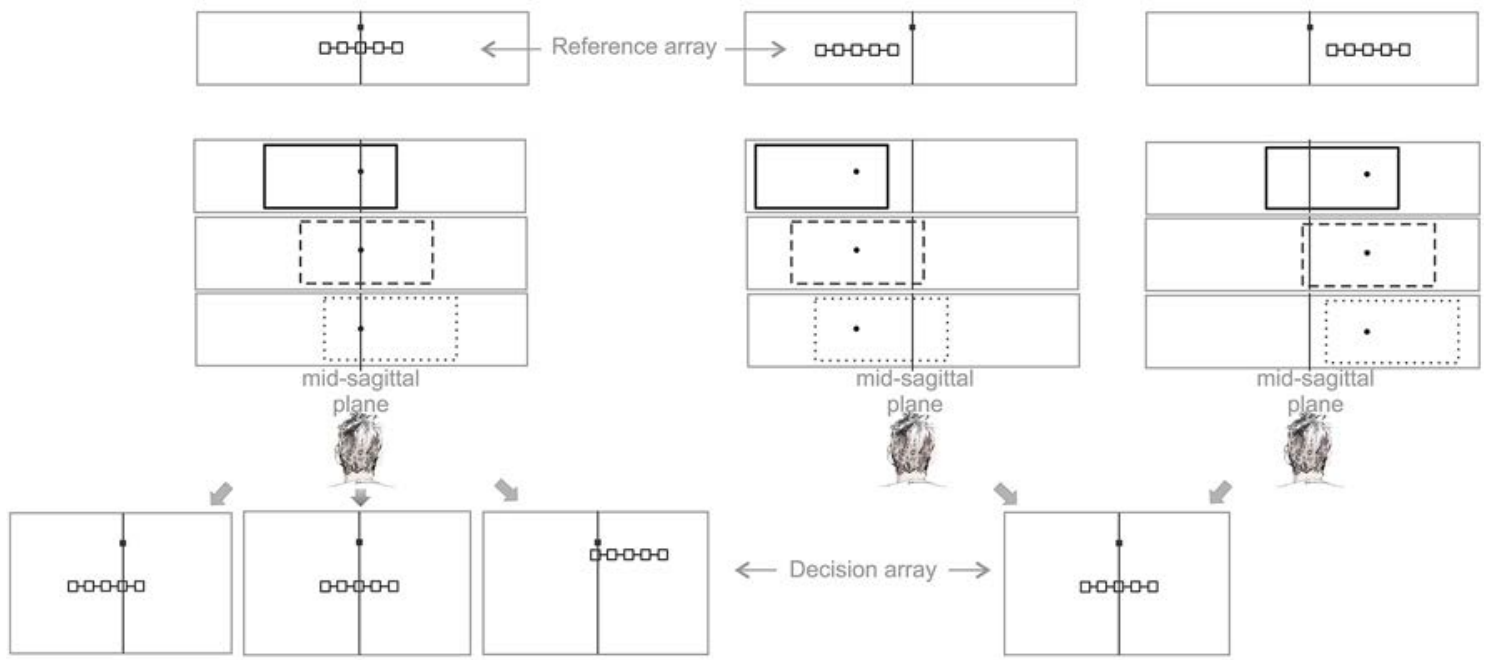

FIGURE 2 | Allocentric IRE task. (A) Following successful eye and hand fixation, subjects are briefly presented a reference array (RA) consisting of five boxes indicating five potential positions for the upcoming cue. After a fixed memory period the cue (dot) was displayed simultaneously with a task-irrelevant context stimulus (frame). Subjects had to compare the position of the cue with the memorized reference positions indicated by the RA boxes to identify and reach to the corresponding target box within a decision array (DA) presented shortly afterwards. The DA was identical to the RA in size and shape but could appear at spatial locations congruent or incongruent to the RA. The vertical line within each frame indicates the subject's mid-sagittal plane. (B) Experiment I: In order to test the IRE in an allocentric reference

position ("spatially not immediate", Experiment I), or only with congruent target position as in previous experiments (Experiment $\mathrm{Ib}$ ). It is important to note that the positions of the frame stimulus relative to the body are still at the straight-ahead direction as in the original Roelofs experiment and in previous IRE experiments. To be able to later dissociate the frame position from any egocentric frame of reference, body-centered or eye-centered, we also tested for the effect of ocular fixation in our task (control frame, we disentangled the position of the RA and DA for two-thirds of the trials. The congruency of the RA-DA was unpredictable to subjects in each trial. Therefore, to perform the task correctly, subjects had to encode the cue relative to the RA, i.e., use object-based (allocentric) spatial encoding. (C) Experiment II: In order to directly test the biased-midline hypothesis we disentangled the position of the RA from subject's objective straight-ahead by randomly displaying the RA in either hemifield. The frame could take three different positions relative to the RA (allocentric shift of the frame to left, right or centered) for each RA location while it remained at the same side relative to the SSA (egocentric shift of the frame to the left/right for RA left/right location). 
the order of a few $10 \mathrm{~ms}$ (Gail and Andersen, 2006; Westendorff et al., 2010; Thaler and Goodale, 2011b; Westendorff and Gail, 2011), such short additional latencies are about two orders of magnitude less than the instructed delays necessary to evoke an IRE in previous experiments (Bridgeman et al., 1997; Dassonville and Bala, 2004b; Dassonville et al., 2004; Walter and Dassonville, 2006, 2008; Bridgeman and Hoover, 2008).

\section{Methods of experiment I}

In experiment I subjects were required to reach-to-touch the target location. The important difference of our design compared to previous IRE studies was that the physical positions of cue and target were spatially congruent in only $1 / 3$ of the trials (Figure $2 B$ ). In the other trials the reference and DA were (partially) incongruent in their position, but otherwise identical. In experiment I the RA position was constant across trials and always at the center of the screen. The DA randomly took one of three possible positions relative to the RA: identical (congruent condition), shifted by $1.5 \mathrm{~cm}\left(2^{\circ}\right)$ to the left (partly congruent), or shifted by $2.12 \mathrm{~cm}$ $\left(2.8^{\circ}\right)$ to the right and $2.12 \mathrm{~cm}$ up (incongruent). Only in the congruent condition were cue (one of the RA boxes) and target (the corresponding DA box) physically identical, as in previous IRE experiments using egocentric reaching or pointing tasks (Bridgeman et al., 1997, 2000; de Grave et al., 2002; Dassonville and Bala, 2004a,b; Dassonville et al., 2004; Walter and Dassonville, 2006, 2008; Bridgeman and Hoover, 2008; Lester and Dassonville, 2011). This task design resulted in 45 possible combinations of cue (target), frame and DA positions $(5 \times 3 \times 3)$, which were randomly presented. Since subjects could not predict whether a trial will be congruent or not, they always had to encode cue position with respect to the RA in order to be able to perform the task correctly. Subjects needed to perform 200 hit trials, resulting in at least four repetitions per condition. In case subjects' errors might not be balanced across conditions, we decided against using "pseudo-random" trial orders to avoid changing probabilities of individual task conditions. Instead, we presented more than $4 \times$ 45 trials to yield a minimum of four repetitions per conditions. Analysis of exactly four trials per condition instead of 4-5 trials per condition did not change the results.

Training was identical to the experimental task, except that the frame was not presented. Training was terminated after 20 hit trials.

\section{Methods of control experiment la}

In a control experiment Ia, we tested whether the presentation of the ocular fixation target has an impact on the IRE. Since previous studies on IRE purposefully tried to avoid any possibility of allocentric spatial coding, no ocular fixation stimulus was shown to subjects during the trial (Dassonville and Bala, 2004b). Hence, in our control experiment Ia, we omitted the ocular fixation stimulus and did not impose any constraints on eye movements. This control was run for all subjects of experiment I on a separate day.

\section{Methods of control experiment Ib}

In control experiment Ib, we reproduced the original IRE paradigm (Bridgeman et al., 1997) in order to establish that our setup and task layout allows us to reproduce previous findings of no IRE in immediate reaches. We used an independent group of subjects to avoid a possible transfer of response strategy between the two experimental designs. Each trial started with ocular and manual fixation. After valid fixation, cue and frame were simultaneously presented. Following the offset of cue and frame, an acoustic go signal indicated to the subjects to lift their finger from the starting home button and reach-to-touch the perceived location of the cue. Subjects had $1000 \mathrm{~ms}$ to finish the reach and they were required to hold ocular fixation until the end of the trial. There were no reference or decision objects shown in control experiment Ib. Importantly, the spatial layout and timing of the stimuli was otherwise identical to experiment I, i.e., the same cue, target and frame positions, sizes and presentation times were used. The 15 different possible combination of cue and frame ( 5 cue locations $\left(0, \pm 2^{\circ}\right.$ and $\pm 4^{\circ}$ relative to the midsagittal plane) and 3 frame locations ( 0 and $\pm 5^{\circ}$ relative to the mid-sagittal plane)) were randomly presented to the subjects. For six out of the nine subjects stimuli had $23.5 \mathrm{~cd} / \mathrm{m}^{2}$ luminance on a $1.2 \mathrm{~cd} / \mathrm{m}^{2}$ background, for the other three the contrast was identical to experiment I. The results were independent of stimulus contrast, hence will be presented jointly.

\section{EXPERIMENT II}

In experiment II, we tested whether the IRE in experiment I can be explained by a biased perception of the SSA. After we established with experiment I that incongruent reference and DA positions encourage allocentric reach planning and allow an IRE for short latency reaches to the target, we now additionally dissociated the position of the RA from the straight-ahead direction to test explicitly whether the IRE is determined by frame position relative to straight-ahead or relative to the RA.

During the training session for experiment II subjects performed the identical task to the incongruent condition of experiment I, but without the frame stimulus. The goal was to familiarize subjects with the setup and the allocentric reach task. Training was terminated after 20 hit trials.

\section{Methods of control experiment Ila}

Trials in experiment IIa were identical to the incongruent condition of experiment I. Subjects conducted 75 correctly performed trials to test whether they were prone to IRE in the allocentric reach task. This served as baseline for the expected effect size in the experiment II for this group of subjects.

\section{Methods of experiment II}

In Experiment II, we dissociated the position of the RA from the objective straight-ahead (see Figure 2C). Except for the positions of decision and RA, the procedure was the same as in the experiment I. The RA was randomly shifted by $5.8^{\circ}(4.5 \mathrm{~cm})$ either to the left or to the right of the objective straight-ahead with equal probability. As an example, consider the case when the RA was shifted to the right by $5.8^{\circ}$. Even if the frame was shifted by the maximum value of $5^{\circ}$ to the left relative to the center of the RA (leftward allocentric shift of the frame), the center-of-mass of the frame still remained in the same hemi-field relative to objective straight-ahead (rightward egocentric shift of 
the frame, see Figure 2C). Although the frame could take three different positions relative to each of the two RA positions, it always stayed to the right of the body's midline if the RA was on the right side, and it stayed left of the body's midline when the RA was on the left side. Subjects were asked to maintain ocular fixation on the fixation target at the objective straight-ahead direction to align the body-centered reference frame with the gaze-centered reference frame. The DA was always located at the center of the screen, i.e., at the objective straight-ahead direction in all trials. According to the biased midline hypothesis, an offcentered frame relative to the body midline will cause target mislocalization to the direction opposite the frame shift. Therefore, one would expect when the RA and the frame were placed in the left or right hemi-field, they would cause a shift of the SSA to the same direction as the egocentric shift of the frame, thereby causing mislocalization of cue or target to the opposite side (Figure 5A). The 30 possible combinations of target, frame and RA positions $(5 \times 3 \times 2)$ were presented in random order. The experiment included 160 hit trials to achieve $4-5$ repetitions per condition.

\section{DATA ANALYSIS}

For each combination of target, frame and DA position, the horizontal reach endpoint relative to the center of the decision array (HRDA) was taken as the subject's response (averaged across 4-5 identical trials). A HRDA of $2^{\circ}(1.5 \mathrm{~cm})$ means that the subject in this condition on average reached $1.5 \mathrm{~cm}$ to the right of the center of the DA. If the central box was cued, a HRDA of $2^{\circ}(1.5 \mathrm{~cm})$ corresponds to the nearest right neighboring box. A two-factor analysis of variance with cue position (5 levels) and frame position (3 levels) as factors was applied to HRDA for the population of all subjects (repeated measures ANOVA). A significant main effect of the factor "frame" indicated IRE. Additionally, for each position of the DA, the HRDA in the frameright conditions was subtracted from the frame-left conditions for each target position and the mean difference was computed. This average localization error was used to compare effect sizes between different task conditions.

\section{RESULTS}

\section{RESULTS OF EXPERIMENT I}

Figures 3A-C shows the average target localization error, quantified by the mean HRDA (see Section Methods), across all 11 subjects. The three panels show separately the three different DA positions. All three DA conditions showed highly significant main effects of the factors "cue" (incongruent/partlycongruent/congruent: $F_{\text {cue }}(4,40)=134 / 124 / 142$, all $p_{\text {cue }}<$ 0.0001 ) and "frame" (incongruent/partly-congruent/congruent:

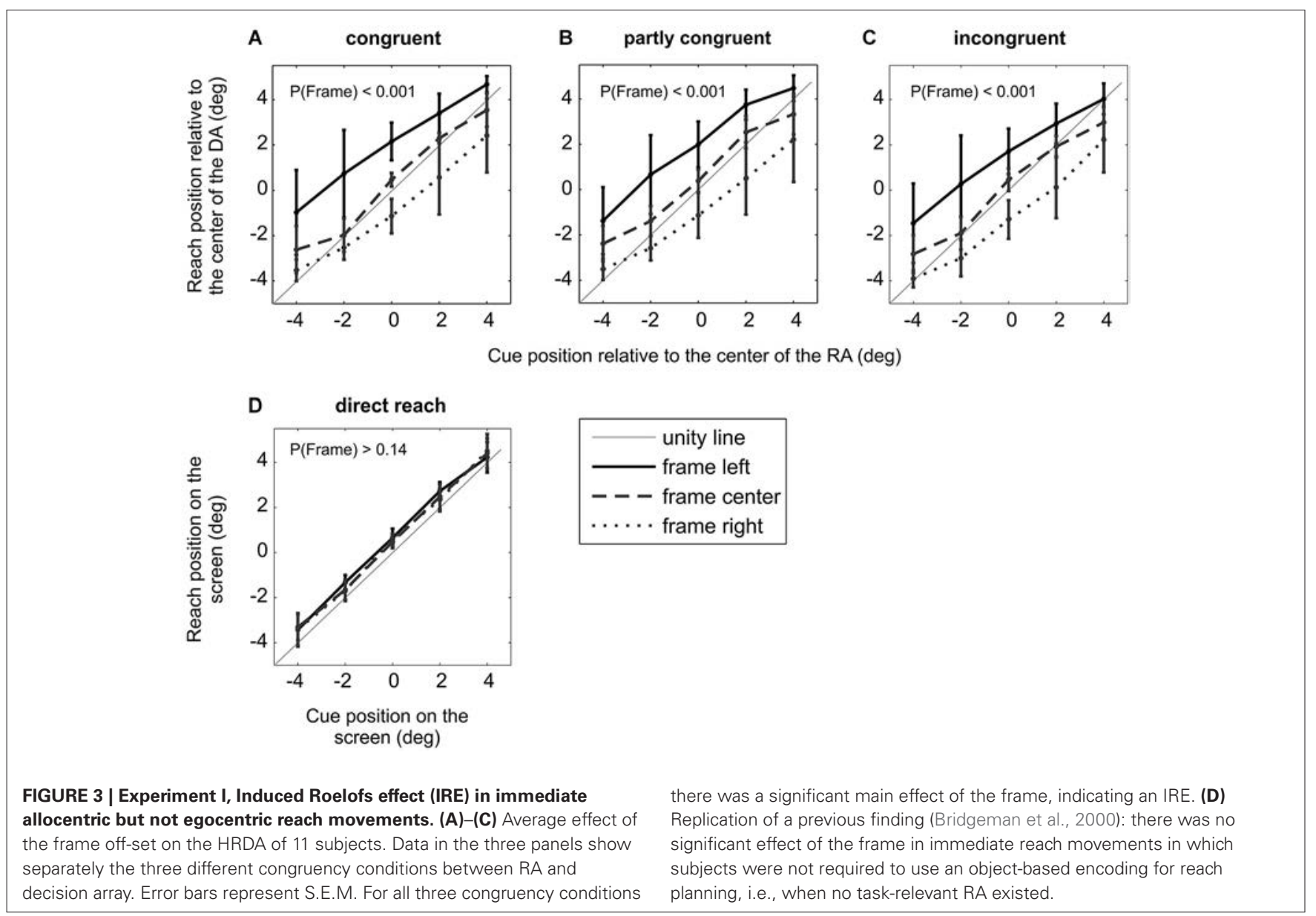




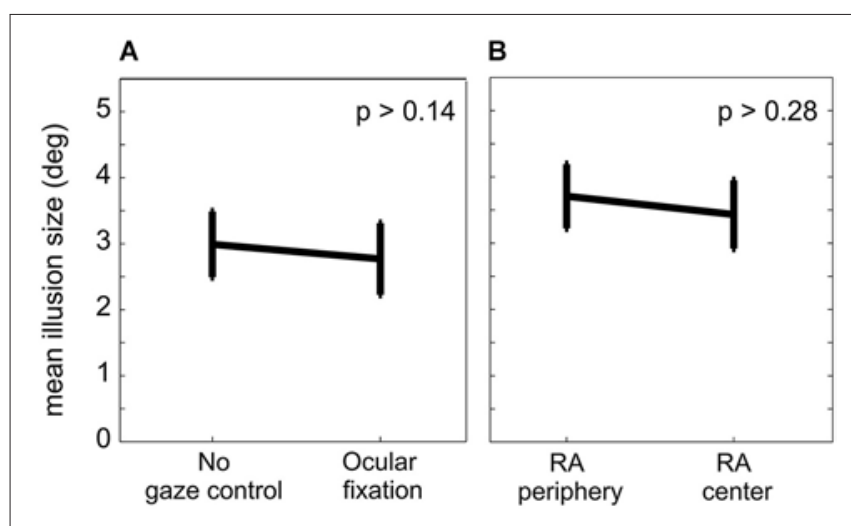

FIGURE 4 | Localization error in different conditions. (A) Mean localization error across 11 subjects and three different congruency conditions with (experiment I) and without (control experiment la) ocular fixation. There was no significant difference between the mislocalization error between the two fixation conditions. (B) Mean localization error across 10 subjects for two lateral positions of RA. There was no significant difference in the mislocalization error between the RA in the periphery (experiment II) and in the center (control experiment Ila). Error bars represent S.E.M.

$F_{\text {frame }}(2,20)=22.6 / 26.5 / 26.7$, all $\left.p_{\text {frame }}<0.0001\right)$, qualified by significant interactions (incongruent/partly-congruent/congruent: $F_{\text {cue }} \times$ frame $(8,80)=5.80 / 6.02 / 5.55$, all $p_{\text {cue }} \times$ frame $\left.<0.0001\right)$.

The significant factor "frame" in all three DA conditions demonstrates that the IRE occurred independently of the trialby-trial level of congruency between the reference and DA. The average localization error for individual subjects shows that the IRE was characterized by varying effect strength with most but not all subjects showing an IRE at the single subject level (average localization error for individual subjects: $3.79^{\circ}, 4.38^{\circ}, 0.52^{\circ}, 1.19^{\circ}$, $\left.5.02^{\circ}, 0.91^{\circ}, 4.20^{\circ}, 2.03^{\circ}, 4.65^{\circ}, 0.29^{\circ}, 3.50^{\circ}\right)$. The congruency condition did not affect the size of the localization error $(p>$ $0.10, F_{\text {congruency }}=2.57$, two-factor repeated measure ANOVA on localization error for population of 11 subjects with factors "congruency" and "target relative to DA"). At the population level, the localization error was $2.77^{\circ}$ (S.E.M. across subjects: $0.54^{\circ}$, S.E.M across all subjects and task conditions: $0.15^{\circ}$; Figure $4 \mathrm{~A}$ ).

This means that even in the congruent condition, which was identical to previous experiments in terms of spatial congruency of cue and reach target, a significant IRE was induced for immediate reaches. This was not the case in previous studies (Bridgeman et al., 1997, 2000; Dassonville and Bala, 2004b; Dassonville et al., 2004; Lester and Dassonville, 2011) where only congruent trials were presented (see also Section Results of control experiment Ib). None of the subjects showed a significant effect of congruency condition on reaction times $(0.20<p<0.97$, one-way ANOVA on per-subject trial-by-trial reaction times with factor "congruency"). From experiment I we can conclude that object-centered allocentric planning of immediate reaches is subject to the IRE.

In control experiment Ia we tested the effect of ocular fixation on the IRE by releasing the eye movement constraints but otherwise keeping everything identical to experiment I. The main result of this control was the same as for experiment I. The three congruency conditions in experiment Ia showed significant main effects of factors "cue" (incongruent/partlycongruent/congruent: $F_{\text {cue }}(4,40)=98.4 / 97.0 / 99.7$, all $p_{\text {cue }}<$ 0.0001 ) and "frame" (incongruent/partly-congruent/congruent: $F_{\text {frame }}(2,20)=38.2 / 32.2 / 34.4$, all $\left.p_{\text {frame }}<0.0001\right)$, qualified by significant interactions (incongruent/partly-congruent/congruent: $F_{\text {cue }} \times$ frame $(8,80)=6.53 / 5.94 / 3.10, p_{\text {cue }} \times$ frame $<0.0001 /<0.0001 /$ $<0.005)$. Mean localization errors for individual subjects were $5.45^{\circ}, 6.22^{\circ}, 0.40^{\circ}, 2.77^{\circ}, 6.24^{\circ}, 1.48^{\circ}, 5.51^{\circ}, 2.57^{\circ}, 5.80^{\circ}$, $1.70^{\circ}$ and $5.52^{\circ}$. Across the population of subjects, the localization error was $2.99^{\circ}$ (S.E.M. $0.50^{\circ}$, Figure 4A). A two-tailed paired $t$-test between experiments I and Ia did not show a significant difference in localization error with and without ocular fixation $(p>0.14)$. From experiment Ia we can thus conclude that in our allocentric reach task the introduction of an ocular fixation constraint to align body- and gaze-centered reference frames does not affect the IRE.

In control experiment $\mathrm{Ib}$ we replicated the original finding of Bridgeman et al. (2000) for immediate reaches by asking subjects to reach and touch the perceived location of spatial cues which were presented within a frame (Figure 3D). The two-factor repeated measure ANOVA on the population of nine subjects showed a significant main effect of the factor "cue" $\left(F_{\text {cue }}(4,32)=435, p_{\text {cue }}<0.0001\right)$, but no significant effect of "frame" $\left(F_{\text {frame }}(2,16)=2.15, p_{\text {frame }}>0.14\right)$, qualified by a significant interaction $\left(F_{\text {cue }} \times\right.$ frame $(8,64)=2.27, p_{\text {cue }} \times$ frame $\left.<0.04\right)$. This means that the subjects correctly directed their reaches to the cue position (main effect of cue), but were unaffected by the frame stimulus (no main effect of frame). Correspondingly, mean localization errors for individual subjects were close to zero: $0.23^{\circ}$, $-0.04^{\circ}, 0.17^{\circ}, 0.56^{\circ}, 0.33^{\circ}, 0.12^{\circ},-0.06^{\circ},-0.19^{\circ}, 0.07^{\circ}$. The lack of an IRE for immediate egocentric reaches is comparable with the original finding (Bridgeman et al., 2000).

\section{RESULTS OF EXPERIMENT II}

In experiment I the sustained presence of a visual landmark at the direction of the objective straight-ahead, namely the fixation spot on which subjects kept ocular fixation, did not diminish the IRE. We consider it unlikely that despite continued ocular fixation at the true straight-ahead direction subjects would undergo a substantial shift in SSA. This allowed us to question the previous hypothesis that IRE is due to a temporarily perturbed perception of the SSA direction, an assumption of the biased-midline hypothesis that we want to test in Experiment II.

For both left and right peripheral positions of the RA, experiment II showed a significant effect of the factors "cue" (left/right: $F_{\text {cue }}(4,36)=111 / 87.3$, all $\left.p_{\text {cue }}<0.0001\right)$ and "frame" (left/right: $F_{\text {frame }}(2,18)=54.0 / 58.4$, all $\left.p_{\text {frame }}<0.0001\right)$, with no significant interaction (left/right: $F_{\text {cue }} \times$ frame $(8,72)=1.63 / 1.69, p_{\text {cue }} \times$ frame $>0.13 />0.11$; Figure 5B). Individual subjects had mean localization errors of $5.08^{\circ}, 4.20^{\circ}, 2.54^{\circ}, 6.09^{\circ}, 5.75^{\circ}, 5.91^{\circ}, 6.25^{\circ}$, $6.73^{\circ}$ and $0.34^{\circ}$ in experiment II and $5.47^{\circ}, 4.43^{\circ}, 0.57^{\circ}, 5.66^{\circ}$, $6.52^{\circ}, 5.75^{\circ}, 5.79^{\circ}, 4.46^{\circ}$ and $0.73^{\circ}$ in control experiment IIa. The average localization error across subjects for peripheral RA in experiment II was $3.71^{\circ}$ (S.E.M. $0.48^{\circ}$ ), and $3.43^{\circ}$ (S.E.M. $0.52^{\circ}$ ) for the central RA in control experiment IIa (Figure 4B). A paired two-tailed $t$-test between test and control experiment did not reveal a significant difference $(p>0.28)$. 


\section{A Biased-midline model prediction}

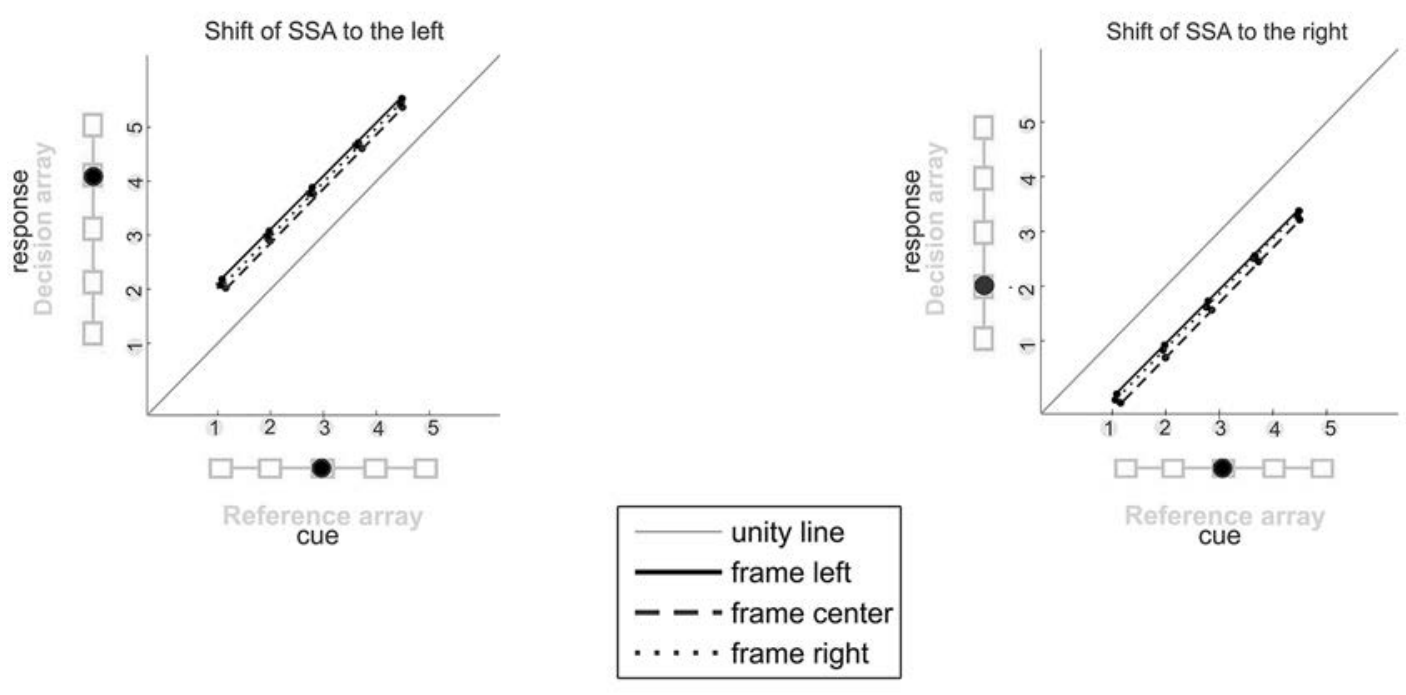

B Result of experiment II

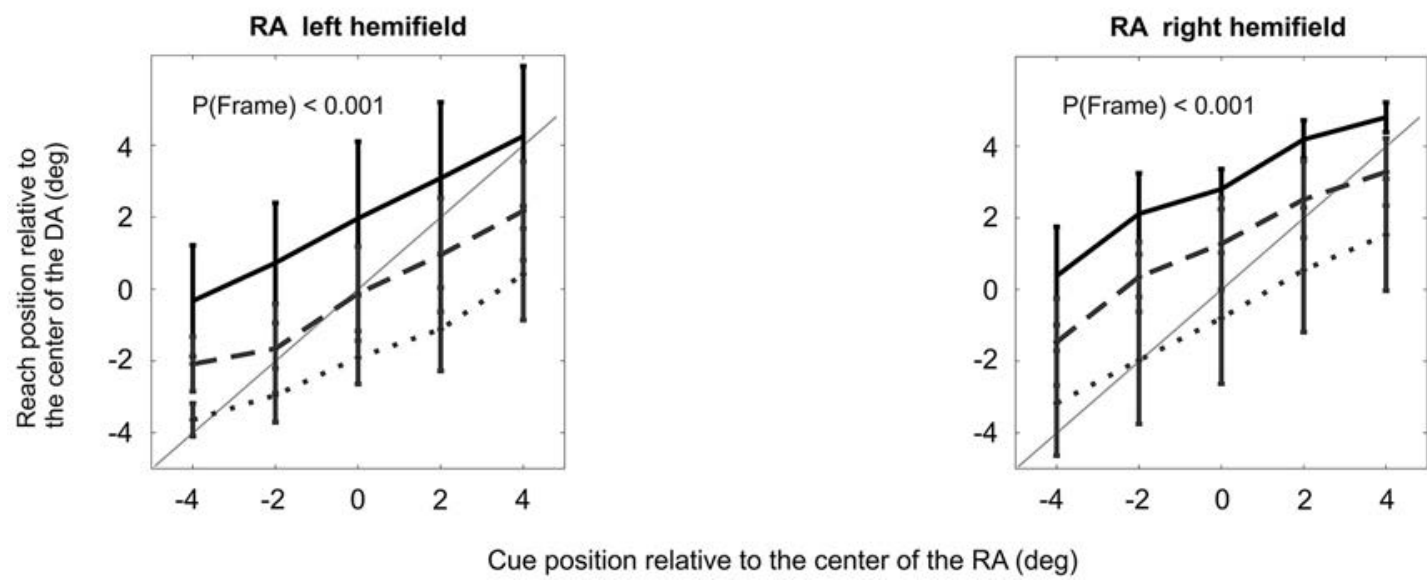

FIGURE 5 | Experiment II behavioral result. (A) According to the biased-midline hypothesis the spatial layout of experiment II would cause mislocalization to the right/left for RA presented in left/right hemifield, respectively. (B) Effect of frame location on the relative average reach endpoint (HRDA) of 10 subjects separately for two positions of the RA indicates that an allocentric shift of the frame (shift relative to the RA) explains the mislocalization best. There was a significant main effect of frame and target location and no significant (frame $\times$ target) interaction.
The result of experiment II shows that the main source of IRE in our data is the relative position of the frame with respect to the reference object (allocentric shift of the frame) rather than with respect to the straight-ahead direction (egocentric shift of the frame).

\section{DISCUSSION}

We conducted two experiments to study the effect of visual contextual information on reach planning. Our two experiments were designed such that subjects were required to encode first the cue and then the reach target relative to the position of a reference object, i.e., in an allocentric reference frame. In this case, subjects reliably showed an IRE (i) even for short-latency reaches to the target; (ii) with and without ocular fixation; and (iii) with mislocalizations being dependent on the allocentric position of the context stimulus (frame) relative to the center of the reference object, not the egocentric position relative to the SSA. Our results are not consistent with a previously suggested biased-midline hypothesis (Dassonville and Bala, 2004b). Instead, we suggest that the IRE can be induced by egocentric or allocentric spatial information, depending on which reference frame the task requires for the behavioral response.

\section{IRE FOR ALLOCENTRIC REACH PLANNING}

In our study we show that IRE can be observed in an allocentric reference frame for reach planning, while previous studies emphasized the role of egocentric reference frames as an explanation. 
Our findings argue against the idea that the IRE in our data can be explained by a phasic shift of the SSA direction (egocentric reference frame), as suggested previously (Dassonville and Bala, 2004b). First, we observed IRE with short-latency reaches. According to the biased-midline hypothesis short-latency reaches should not be subject to IRE since the assumed shift of the SSA is only phasic and affects target localization and reach planning likewise, such that the effect cancels out after relaxation of the SSA perturbation. Second, the fact that in our experiment I the IRE was also present when subjects were required to keep ocular fixation at a visual spot in the objective straight-ahead direction provided an additional hint that a shift in SSA might not be the cause of our observed results. We consider it rather unlikely that the SSA shifts in response to presentation of an off-center visual frame while subjects are fixating at a stable landmark in the true straight-ahead direction. Third, our experiment II provided direct evidence against the biased-midline hypothesis. For task conditions which should all have induced a SSA shift in the same direction, we found IRE in opposite directions (Figure 5). We therefore argue that in our data the Roelofs effect was not induced by an effect of the contextual visual frame stimulus on the SSA.

Ruling out a shifted SSA as explanation of the IRE in our experiment brings up the question which other egocentric or allocentric spatial encoding might be responsible for the observed IRE. Previous results do not contradict the idea of an allocentric IRE, since egocentric and allocentric reference frames were typically not dissociated. In previous IRE experiments (Bridgeman et al., 1997, 2000; de Grave et al., 2002; Dassonville and Bala, 2004a,b; Dassonville et al., 2004; Walter and Dassonville, 2006, 2008; Bridgeman and Hoover, 2008; Lester and Dassonville, 2011) subjects memorized the potential cue positions during a primary training period or behavioral calibration (i.e., equivalent to presentation of the RA in the present experiment). Later in the experiment or later in the trial subjects were asked to conduct reaches or saccades in which the egocentric encoding of the cue location was sufficient to solve the task. When subjects did not need to use the memorized positions to determine the target, no IRE was observed for immediate responses. But in such a task design, egocentric and allocentric references are aligned and the task-irrelevant visual frame is off-set equally in both reference frames. Therefore, even previous IRE task designs which required subjects to conduct a movement directly aiming at the target position, can in principle be consistent with an allocentric cause. Egocentric and allocentric representations of space are present in parallel and both types of information are usually used for more accurate behavior (Burgess, 2006; Byrne and Crawford, 2010). It has been shown that egocentric spatial memory is short lasting, putatively because it has to provide mainly real-time representation of the environment for direct interaction with objects (Hay and Redon, 2006; Chen et al., 2011). The fact that in previous task designs IRE was observed after a certain delay could be attributed to the interaction of shortlasting egocentric and long-lasting allocentric spatial representations. When the same subjects were exposed to a symbolic version of the task in which they had to use the memorized reference positions for a verbal response (to compare the position of visual cue with the memorized array of reference positions and report which one was cued), then the IRE was present even in immediate responses (Bridgeman et al., 1997; de Grave et al., 2002; Dassonville and Bala, 2004b). We argue that the verbal report of cue position required subjects to encode the cue relative to the RA hence mandated the use of an allocentric reference frame. It is therefore possible that even in previous IRE experiments the allocentric offset of the frame was the source of the illusion.

We suggest that the IRE in our reach task at least partially depended on allocentric encoding of space. Our present experimental design required subjects to follow an object-centered, hence allocentric, movement planning strategy. For proper interpretation of the IRE it is necessary to distinguish different phases of the trial when discussing reference frames. In the context of our task, at least the following spatial parameters are of interest: (i) the ego- or allocentric position of the (memorized) RA; (ii) the egocentric position of the frame relative to the bodymidline; (iii) the allocentric position of the frame relative to the RA; (iv) the allocentric position of the cue relative to the memorized RA; and (v) the allocentric target position relative to the DA. Experiment II showed that the IRE was determined by the allocentric frame position relative to the RA, not the frame's egocentric position. Thus, the IRE had an allocentric cause in our case. The consequence of this original allocentric cause needs to survive or be inherited by subsequent spatial encoding steps in order to affect the final motor behavior. The question is, which spatial encoding mediates the originally allocentric effect to finally become apparent in allocentric reach behavior? We ruled out a shifted SSA above. Previous studies showed that the memorized location of the reference object is shifted by the frame stimulus (Dassonville and Bala, 2004b). In our case this subjective shift of the RA would be sufficient to explain the results. The subjects need to encode the cue relative to the RA and later compute the target as the corresponding position on the relocated reference object (DA). Hence, a shifted RA translates into an erroneous allocentric cue position, and this in turn translates into an erroneous allocentric target position, and finally into an erroneous reach. Whether the memorized RA itself is encoded in an egocentric reference frame (e.g., relative to direction of gaze or body midline) or in an allocentric reference frame (e.g., relative to the surrounding screen frame) does not matter for the outcome. Both are possible and our experiment did not dissociate these alternatives.

\section{EXPANSION OF MEMORIZED VISUAL SPACE}

In all previous IRE studies, an underestimation of target eccentricity was reported along with a significant systematic mislocalization of the target. This can be seen by the fact that movement endpoint position as a function of cue position has a slope smaller than unity. The present results (Figures 3 and 5) also show underestimation of target eccentricity $\left(p_{\text {experiment I }}<0.0001\right.$ and $p_{\text {experiment II }}<0.0001$, one tail $t$-test on the slope of the nine regression lines fitted separately to the population response for different DA and frame positions in experiments I and six regression lines fitted separately to the population response for different RA and frame positions in experiments II). In contrast to previous 
reports, subjects underestimated the object-centered eccentricity of the cue or target (i.e., laterality of the cue/target relative to the center of the reference/DA). Underestimated eccentricity can be viewed as an apparent compression of the movement space. Yet, when in a previous study subjects were asked to make saccadic eye movements to memorized reference locations, the apparent compression turned out to be a result of expansion of the spatial memory of potential target positions (Dassonville and Bala, 2004a). Our observed underestimation of eccentricity adds to previous findings by showing that expansion of memorized visual space occurs in the behaviorally relevant reference frame, here centered on the object.

\section{PERCEPTION VS. ACTION}

We do not argue that the behavioral report via an allocentric reach is necessarily substantially different from IRE tasks with symbolic encoding of the target, e.g., by key-presses or verbal report. The underlying mechanism of the IRE for this class of tasks, which previously were labeled "perceptual", might be identical or at least overlap. Accordingly, previous lines of argumentation based on a perception-action model might also account for our data (see also Section Discussion on ventral and dorsal stream processing below). In this case, we would have to assume that the memorized RA underwent a "perceptual" shift due to the context stimulus (Dassonville and Bala, 2004b) with the consequences discussed in the above paragraph. Whether allocentric reach planning and perceptual encoding in the context of such tasks can at all be meaningfully distinguished, remains an open question. We find it noteworthy, though, that the congruent condition of the allocentric task (Experiment I, congruent trials) and the egocentric control condition (Experiment $\mathrm{Ib}$ and previous studies), were equivalent in terms of spatial layout, timing of stimuli, and manual response mode, and only differed in the task context requiring allocentric reach target selection. In terms of spatial layout, the equivalency refers to the fact that in congruent trials, the allocentric and egocentric spatial location of the cue (the dot which is presented with the frame) and the target (final reach goal) are identical. In terms of timing, equivalency refers to the fact that in both experiments subjects receive the acoustic gosignal soon after the presentation of the cue plus frame stimulus and faster than typical manual response times would require. In this sense the immediacy of the movement is given in both experiments. The task context was not provided by the congruent trials themselves but rather by the interspersed incongruent trials which requested subjects to encode the cue relative to the RA rather than according to their liking. If the congruent condition would have been predictable, the congruent trials could have been solved with egocentric encoding of the cue and target. This rendered the allocentric congruent trials, which showed an IRE, quite similar to the egocentric trials, which did not show an IRE. This means that spatial task context was enough to make shortlatency reaches, which share many properties of typical "action" tasks, prone to IRE.

The results of experiment I differ from a recent study on IRE with an allocentric task in which the stimuli defining the allocentric reference frame were shown simultaneously with the off-center context stimulus, and no visual cue was shown to instruct the target (Dassonville and Bala, 2004b). The reach target was inferred in an allocentric reference frame as the fourth corner of a rectangle, while the other three corners were presented within a visual frame stimulus shifted laterally relative to the subject's mid-sagittal plane. The pattern of observed target errors was similar to previous IRE experiments, with no effect for immediate responses and a significant effect for delayed responses. When the reference stimuli were shown together with an off-center frame, one had to expect that they will be subject to an IRE themselves (Lester and Dassonville, 2011) and the mislocalization of the target, which has to be inferred from the affected reference objects, is then a secondary effect without an IRE on the allocentric space representation itself. These results were used to argue against separate cognitive and sensorimotor visuospatial representations, and were instead explained with the biased-midline hypothesis, i.e., by an egocentric cause, an explanation that does not work for our data.

Taken together, we conclude that in our task, which required reach planning in an allocentric reference frame, the IRE was caused by an allocentric space representation and mediated via a distorted visual memory of the reference object. This may also have been the case in previous Roelofs experiments. It cannot be ruled out that an egocentric mislocalization of the memorized RA gave rise to the allocentric mislocalization of the visual cue, but it can be ruled out that the original cause for the mislocalization was a shift of the SSA direction or any other egocentric reference frame.

\section{VENTRAL VS. DORSAL VISUAL STREAMS}

According to the perception-action model (Goodale and Westwood, 2004; Goodale et al., 2004), egocentric references support visually guided actions through the dorsal sensorimotor stream in the posterior parietal cortex, while allocentric encoding of spatial locations can be predominantly found in the ventral stream supporting perception. According to this view, the dorsal stream is required and capable of making use of allocentric information from the ventral stream in the case of memory guided movements, e.g., IRE pointing tasks with long delays (Bridgeman et al., 1997; Goodale and Westwood, 2004; Milner and Goodale, 2008). In terms of the short-latency manual interaction with the visual target stimulus, our task would have to be considered a typical "action" task, hence should be attributed to dorsal stream processing. But according to the perception-action model, the allocentric spatial task constraints in our task also require ventral stream input. The model does not provide threshold values of how quickly ventral stream information can become accessible to dorsal stream processing. But in previous experiments, the required delays in target-aiming pointing, reaching, or saccade tasks ranged in the order of seconds before an IRE became apparent, suggesting a very slow transfer of information between ventral and dorsal stream in IRE tasks. If the model does account for our data, then our results suggest that the use of allocentric ventral-stream information by dorsal stream visuomotor processing can occur much faster than thought from previous IRE experiments. Such fast transfer is also suggested by a recent behavioral study (Thaler and Goodale, 2011b) which showed that reaction times in allocentric movements are 30-40 ms slower than 
egocentric movements, a finding that is reminiscent of behavioral and neural response delays in posterior parietal cortex during stimulus-response incongruent reach tasks (Gail and Andersen, 2006; Westendorff et al., 2010; Westendorff and Gail, 2011).

Slow brain imaging techniques cannot resolve the issue of whether such short-latency ventral-to-dorsal information transfer occurs, but experimental results have repeatedly pointed to overlapping structures for egocentric and allocentric encoding in the dorsal stream (Galati et al., 2000; Zaehle et al., 2007; Thaler and Goodale, 2011a; Gallivan et al., 2013). From our own previous neurophysiology work, we know that posterior parietal cortex encodes memory-guided anti-reach goals, which are independent of immediate visual input and independent of visual memory, with a delay of roughly $200 \mathrm{~ms}$ relative to visual cue onset, and roughly $100 \mathrm{~ms}$ relative to the visually selective neural response onset in the same area (Gail and Andersen, 2006; Gail et al., 2009; Westendorff et al., 2010). From the above discussion, we expect similar latencies for allocentric encoding in the posterior parietal cortex in the context of the current task.

The extent to which the perception-action model is valid is an ongoing debate in visual and visuomotor neuroscience. Growing evidence from behavioral and neurophysiology studies challenges the strictly separated vision-for-perception and visionfor-action theory (see Schenk et al., 2011 for review). The most compelling evidence for this model was patient D.F., who has bilateral damage to the ventral stream. D.F. failed in visual perceptual tasks while her visuomotor performance was not impaired (Milner et al., 1991). A recent study (Schenk, 2006) revealed that the discrepancy in her behavior was not due to different response modes, but rather due to deficits in different spatial representations (Himmelbach et al., 2012). The study showed that her perceptual performance was as good as her visuomotor performance when the perceptual task demanded egocentric spatial encoding whereas she failed in perceptual tasks which required object-based (allocentric) spatial encoding. Further behavioral support for the perception-action model was provided by a substantial body of research exploring visual illusions in perceptual and motor tasks where unlike perceptual responses, immediate motor responses seemed to be robust to the erroneous effects of spatial contextual information (for recent reviews see Schenk et al., 2011; Westwood and Goodale, 2011). However, in more controlled experimental conditions, contextual information can similarly affect perceptual and motor responses (Glover, 2004; Coello et al., 2007; Neely et al., 2008; Schenk et al., 2011). Therefore, based on our IRE for short-latency reaches, we suggest that the differential effect of spatial contextual information on sensorimotor behavior as explained by the perception-action model might not primarily be a question of perceptual vs. actionlike behavioral response mode, but rather a question of the spatial task demands.

\section{ACKNOWLEDGMENTS}

We thank Diemut Regel and Katharina Wörgötter for help in data collection, Sina Plümer and Klaus Heisig for administrative and technical support. This work was supported by the Federal Ministry for Education and Research (BMBF, Germany, grants, 01GQ0814 and 01GQ 1005C), the German Research Foundation
(DFG, grant SFB-889), and the State of Lower Saxony (grant VWZN2563).

\section{REFERENCES}

Andersen, R. A., Essick, G. K., and Siegel, R. M. (1985). Encoding of spatial location by posterior parietal neurons. Science 230, 456-458. doi: 10.1126/science. 4048942

Bridgeman, B., Gemmer, A., Forsman, T., and Huemer, V. (2000). Processing spatial information in the sensorimotor branch of the visual system. Vision Res. 40, 3539-3552. doi: 10.1016/s0042-6989(00)00193-0

Bridgeman, B., and Hoover, M. (2008). Processing spatial layout by perception and sensorimotor interaction. Q. J. Exp. Psychol. (Hove) 61, 851-859. doi: 10. 1080/17470210701623712

Bridgeman, B., Peery, S., and Anand, S. (1997). Interaction of cognitive and sensorimotor maps of visual space. Percept. Psychophys. 59, 456-469. doi: 10. 3758/bf03211912

Burgess, N. (2006). Spatial memory: how egocentric and allocentric combine. Trends Cogn. Sci. 10, 551-557. doi: 10.1016/j.tics.2006.10.005

Byrne, P. A., and Crawford, J. D. (2010). Cue reliability and a landmark stability heuristic determine relative weighting between egocentric and allocentric visual information in memory-guided reach. J. Neurophysiol. 103, 3054-3069. doi: 10. 1152/jn.01008.2009

Chafee, M. V., Averbeck, B. B., and Crowe, D. A. (2007). Representing spatial relationships in posterior parietal cortex: single neurons code object-referenced position. Cereb. Cortex 17, 2914-2932. doi: 10.1093/cercor/bhm017

Chen, Y., Byrne, P., and Crawford, J. D. (2011). Time course of allocentric decay, egocentric decay and allocentric-to-egocentric conversion in memory-guided reach. Neuropsychologia 49, 49-60. doi: 10.1016/j.neuropsychologia.2010.10.031

Coello, Y., Danckert, J., Blangero, A., and Rossetti, Y. (2007). Do visual illusions probe the visual brain? Illusions in action without a dorsal visual stream. Neuropsychologia 45, 1849-1858. doi: 10.1016/j.neuropsychologia.2006. 12.010

Crowe, D. A., Averbeck, B. B., and Chafee, M. V. (2008). Neural ensemble decoding reveals a correlate of viewer- to object-centered spatial transformation in monkey parietal cortex. J. Neurosci. 28, 5218-5228. doi: 10.1523/jneurosci.5105-07. 2008

Dassonville, P., and Bala, J. K. (2004a). Are the original Roelofs effect and the induced Roelofs effect confounded by the same expansion of remembered space? Vision Res. 44, 1025-1029. doi: 10.1016/j.visres.2003.10.018

Dassonville, P., and Bala, J. K. (2004b). Perception, action and Roelofs effect: a mere illusion of dissociation. PLoS Biol. 2:e364. doi: 10.1371/journal.pbio. 0020364

Dassonville, P., Bridgeman, B., Kaur, B. J., Thiem, P., and Sampanes, A. (2004). The induced Roelofs effect: two visual systems or the shift of a single reference frame? Vision Res. 44, 603-611. doi: 10.1016/j.visres.2003.10.017

de Grave, D. D., Brenner, E., and Smeets, J. B. (2002). Are the original Roelofs effect and the induced Roelofs effect caused by the same shift in straight ahead? Vision Res. 42, 2279-2285. doi: 10.1016/s0042-6989(02)00189-x

Fink, G. R., Marshall, J. C., Weiss, P. H., Stephan, T., Grefkes, C., Shah, N. J., et al. (2003). Performing allocentric visuospatial judgments with induced distortion of the egocentric reference frame: an fMRI study with clinical implications. Neuroimage 20, 1505-1517. doi: 10.1016/j.neuroimage.2003.07.006

Gail, A., and Andersen, R. A. (2006). Neural dynamics in monkey parietal reach region reflect context-specific sensorimotor transformations. J. Neurosci. 26, 9376-9384. doi: 10.1523/jneurosci.1570-06.2006

Gail, A., Klaes, C., and Westendorff, S. (2009). Implementation of spatial transformation rules for goal-directed reaching via gain modulation in monkey parietal and premotor cortex. J. Neurosci. 29, 9490-9499. doi: 10.1523/jneurosci.109509.2009

Galati, G., Lobel, E., Vallar, G., Berthoz, A., Pizzamiglio, L., and Le Bihan, D. (2000). The neural basis of egocentric and allocentric coding of space in humans: a functional magnetic resonance study. Exp. Brain Res. 133, 156-164. doi: 10. 1007/s002210000375

Galati, G., Pelle, G., Berthoz, A., and Committeri, G. (2010). Multiple reference frames used by the human brain for spatial perception and memory. Exp. Brain Res. 206, 109-120. doi: 10.1007/s00221-010-2168-8

Gallivan, J. P., Chapman, C. S., McLean, D. A., Flanagan, J. R., and Culham, J. C. (2013). Activity patterns in the category-selective occipitotemporal cortex 
predict upcoming motor actions. Eur. J. Neurosci. 38, 2408-2424. doi: 10.1111/ ejn. 12215

Glover, S. (2004). Separate visual representations in the planning and control of action. Behav. Brain Sci. 27, 3-24. doi: 10.1017/s0140525x04000020

Goodale, M. A., and Milner, A. D. (1992). Separate visual pathways for perception and action. Trends Neurosci. 15, 20-25. doi: 10.1016/0166-2236(92)90344-8

Goodale, M. A., and Westwood, D. A. (2004). An evolving view of duplex vision: separate but interacting cortical pathways for perception and action. Curr. Opin. Neurobiol. 14, 203-211. doi: 10.1016/j.conb.2004.03.002

Goodale, M. A., Westwood, D. A., and Milner, A. D. (2004). Two distinct modes of control for object-directed action. Prog. Brain Res. 144, 131-144. doi: 10. 1016/s0079-6123(03)14409-3

Hay, L., and Redon, C. (2006). Response delay and spatial representation in pointing movements. Neurosci. Lett. 408, 194-198. doi: 10.1016/j.neulet.2006. 08.080

Himmelbach, M., Boehme, R., and Karnath, H. O. (2012). 20 years later: a second look on DF's motor behaviour. Neuropsychologia 50, 139-144. doi: 10.1016/j. neuropsychologia.2011.11.011

Lester, B. D., and Dassonville, P. (2011). Attentional control settings modulate susceptibility to the induced Roelofs effect. Atten. Percept. Psychophys. 73, 13981406. doi: 10.3758/s13414-011-0123-9

Milner, A. D., and Goodale, M. A. (2008). Two visual systems re-viewed. Neuropsychologia 46, 774-785. doi: 10.1016/j.neuropsychologia.2007.10.005

Milner, A. D., Perrett, D. I., Johnston, R. S., Benson, P. J., Jordan, T. R., Heeley, D. W., et al. (1991). Perception and action in "visual form agnosia". Brain 114, 405-428. doi: 10.1093/brain/114.1.405

Neely, K. A., Tessmer, A., Binsted, G., and Heath, M. (2008). Goal-directed reaching: movement strategies influence the weighting of allocentric and egocentric visual cues. Exp. Brain Res. 186, 375-384. doi: 10.1007/s00221-007-1238-Z

Schenk, T. (2006). An allocentric rather than perceptual deficit in patient D.F. Nat. Neurosci. 9, 1369-1370. doi: 10.1038/nn1784

Schenk, T., Franz, V., and Bruno, N. (2011). Vision-for-perception and visionfor-action: which model is compatible with the available psychophysical and neuropsychological data? Vision Res. 51, 812-818. doi: 10.1016/j.visres.2011. 02.003

Smeets, J. B. J., and Brenner, E. (2001). Action beyond our grasp. Trends Cogn. Sci. 5:287. doi: 10.1016/s1364-6613(00)01684-3

Smeets, J. B., Brenner, E., de Grave, D. D., and Cuijpers, R. H. (2002). Illusions in action: consequences of inconsistent processing of spatial attributes. Exp. Brain Res. 147, 135-144. doi: 10.1007/s00221-002-1185-7

Snyder, L. H., Grieve, K. L., Brotchie, P., and Andersen, R. A. (1998). Separate bodyand world-referenced representations of visual space in parietal cortex. Nature 394, 887-891. doi: 10.1038/29777
Thaler, L., and Goodale, M. A. (2011a). Neural substrates of visual spatial coding and visual feedback control for hand movements in allocentric and targetdirected tasks. Front. Hum. Neurosci. 5:92. doi: 10.3389/fnhum.2011.00092

Thaler, L., and Goodale, M. A. (2011b). Reaction times for allocentric movements are $35 \mathrm{~ms}$ slower than reaction times for target-directed movements. Exp. Brain Res. 211, 313-328. doi: 10.1007/s00221-011-2691-2

Vallar, G., Lobel, E., Galati, G., Berthoz, A., Pizzamiglio, L., and Le, B. D. (1999). A fronto-parietal system for computing the egocentric spatial frame of reference in humans. Exp. Brain Res. 124, 281-286. doi: 10.1007/s002210050624

Walter, E., and Dassonville, P. (2006). Fragments of the Roelofs effect: a bottom-up effect equal to the sum of its parts. Percept. Psychophys. 68, 1243-1253. doi: 10. 3758/bf03193724

Walter, E., and Dassonville, P. (2008). Visuospatial contextual processing in the parietal cortex: an fMRI investigation of the induced Roelofs effect. Neuroimage 42, 1686-1697. doi: 10.1016/j.neuroimage.2008.06.016

Westendorff, S., and Gail, A. (2011). What is "anti" about anti-reaches? Reference frames selectively affect reaction times and endpoint variability. Exp. Brain Res. 208, 287-296. doi: 10.1007/s00221-010-2481-2

Westendorff, S., Klaes, C., and Gail, A. (2010). The cortical timeline for deciding on reach motor goals. J. Neurosci. 30, 5426-5436. doi: 10.1523/jneurosci.4628-09. 2010

Westwood, D. A., and Goodale, M. A. (2011). Converging evidence for diverging pathways: neuropsychology and psychophysics tell the same story. Vision Res. 51, 804-811. doi: 10.1016/j.visres.2010.10.014

Zaehle, T., Jordan, K., Wustenberg, T., Baudewig, J., Dechent, P., and Mast, F. W. (2007). The neural basis of the egocentric and allocentric spatial frame of reference. Brain Res. 1137, 92-103. doi: 10.1016/j.brainres.2006.12.044

Conflict of Interest Statement: The authors declare that the research was conducted in the absence of any commercial or financial relationships that could be construed as a potential conflict of interest.

Received: 08 April 2014; accepted: 12 August 2014; published online: 29 August 2014. Citation: Taghizadeh B and Gail A (2014) Spatial task context makes shortlatency reaches prone to induced Roelofs illusion. Front. Hum. Neurosci. 8:673. doi: 10.3389/fnhum.2014.00673

This article was submitted to the journal Frontiers in Human Neuroscience.

Copyright (๑) 2014 Taghizadeh and Gail. This is an open-access article distributed under the terms of the Creative Commons Attribution License (CC BY). The use, distribution or reproduction in other forums is permitted, provided the original author(s) or licensor are credited and that the original publication in this journal is cited, in accordance with accepted academic practice. No use, distribution or reproduction is permitted which does not comply with these terms. 


\title{
Corrigendum: Spatial task context makes short-latency reaches prone to induced Roelofs illusion
}

\author{
Bahareh Taghizadeh ${ }^{1,2}$ and Alexander Gail ${ }^{1,2,3 *}$ \\ ' Sensorimotor Group, Cognitive Neuroscience Lab, German Primate Center, Goettingen, Germany \\ ${ }^{2}$ Faculty of Biology and Psychology, Georg-August-Universität, Göttingen, Germany \\ ${ }^{3}$ Bernstein Center for Computational Neuroscience, Göttingen, Germany \\ *Correspondence: agail@gwdg.de \\ Edited by: \\ J. Douglas Crawford, York University, Canada \\ Reviewed by: \\ Lore Thaler, Durham University, UK \\ Thomas Schenk, Universitätsklinikum Erlangen, Germany
}

Keywords: reach movement, induced Roelofs effect, Illusion, reference frame, allocentric, object-centered

\section{A corrigendum on}

Spatial task context makes short-latency reaches prone to induced Roelofs illusion by Taghizadeh B., and Gail A. (2014). Front. Hum. Neurosci. 8:673. doi: 10.3389/fnhum. 2014.00673

The authors regret errors in the reported subject numbers and values for the illusion size in the data for individual subjects. This affects experiments Ia, Ib, II, and IIa. This mistake does not affect any of the conclusions of the paper since the reported mean values and standard errors were all correct. Correct values for the illusion size in the $N$ individual subjects for each experiment are as follows:
E Ia $(N=11)$ :

$4.13^{\circ} 4.69^{\circ} 0.30^{\circ} 2.08^{\circ} 4.70^{\circ} 1.11^{\circ} 4.15^{\circ}$

$1.93^{\circ} 4.37^{\circ} 1.27^{\circ} 4.16^{\circ}$

$\mathrm{E} \operatorname{Ib}(N=9)$ :

$\begin{array}{lllll}0.17^{\circ}-0.03^{\circ} & 0.13^{\circ} & 0.42^{\circ} & 0.25^{\circ} & 0.09^{\circ}\end{array}$

$-0.04^{\circ}-0.14^{\circ} 0.06^{\circ}$

E II $(N=10)$ :

$3.83^{\circ} 3.16^{\circ} 1.91^{\circ} 4.76^{\circ} 4.59^{\circ} 4.33^{\circ} 4.45^{\circ}$

$4.71^{\circ} 5.07^{\circ} 0.26^{\circ}$

E IIa $(N=10)$ :

$4.12^{\circ} 3.33^{\circ} 0.43^{\circ} 4.69^{\circ} 4.26^{\circ} 4.91^{\circ} 4.33^{\circ}$

$4.36^{\circ} 3.36^{\circ} 0.55^{\circ}$

Conflict of Interest Statement: The authors declare that the research was conducted in the absence of any commercial or financial relationships that could be construed as a potential conflict of interest.

Received: 27 September 2014; accepted: 29 October 2014; published online: 14 November 2014.

Citation: Taghizadeh B and Gail A (2014) Corrigendum: Spatial task context makes short-latency reaches prone to induced Roelofs illusion. Front. Hum. Neurosci. 8:923. doi: 10.3389/fnhum.2014.00923

This article was submitted to the journal Frontiers in Human Neuroscience.

Copyright @ 2014 Taghizadeh and Gail. This is an open-access article distributed under the terms of the Creative Commons Attribution License (CC BY). The use, distribution or reproduction in other forums is permitted, provided the original author(s) or licensor are credited and that the original publication in this journal is cited, in accordance with accepted academic practice. No use, distribution or reproduction is permitted which does not comply with these terms. 


\subsection{Dynamic and scalable object-based spatial selectivity in monkey parietal reach region and dorsal premotor cortex}

When reaching towards an object, the reach goal has to be identified relative to the object, in the object-centered reference frame, as described in the introduction. Since processing of objectcentered spatial relations was usually assigned to the ventral stream (see Introduction), it was not clear whether single cells in the reach planning network (as a part of the dorsal stream) could encode object-centered reference frame. In this study, we addressed object-centered planning of the reach movement in the single cell level. We asked whether neurons in PRR and PMd used object-centered reference frames when a monkey was required to memorize a peripheral visual cue and to plan a reach relative to other objects. We further asked if not only the object position influences the center of reference for spatial selectivity in PRR and PMd, but also if the object size determines the spatial scaling (tuning width) of the neural selectivity.

We found neurons in both areas that encode the position of the visual cue and the reach goal in an object-centered reference frame. We also found neurons with egocentric or intermediate (mixed egocentric and object-centered) reference frames. Results show that a subset of objectcentered neurons scaled the width of their tuning curve to the size of the object. These cells maintain their pattern of selectivity to different locations on the object when the object size changes. 


\title{
Dynamic and scalable object-based spatial selectivity in monkey parietal reach region and dorsal premotor cortex
}

\author{
*B.TAGHIZADEH SARSHOURI ${ }^{1,2}$, A. GAIL ${ }^{1,2,3}$; \\ ${ }^{1}$ Cognitive Neurosci. Lab., German Primate Ctr., Goettingen, Germany; ${ }^{2}$ Georg-August-Universität Göttingen, \\ Göttingen, Germany; ${ }^{3}$ Bernstein Ctr. for Computat. Neurosci., Göttingen, Germany
}

\begin{abstract}
During visually guided reach planning, neurons in monkey parietal reach region (PRR) and dorsal pre-motor cortex (PMd) encode task-relevant spatial information. Neurons in these areas have been shown to encode reach targets in egocentric reference frames, namely, eye-centered; hand-centered; or intermediate eye-hand reference frames. When reaches are directed towards objects, the reach goal has to be identified not only relative to the body of the subject (egocentric) but also relative to the object (object-centered, that is, allocentric). In object oriented reach planning, the object shape and size partly determine the relevant space for motor planning. Here, we asked whether neurons in PRR and PMd used object-centered reference frames when a monkey was required to memorize a peripheral visual cue and to plan a center-out reach relative to other objects. We found that reach goals are encoded in egocentric reference frames, but also relative to the target object (object-centered reference frame) both in PRR and PMd. Results shows that PRR and PMd switch their encoding from predominantly object-centered during the visual memory period to predominantly egocentric during movement planning. We further tested if not only the object position influences the center of reference for spatial selectivity in PRR and PMd, but also if the object size determines the spatial scaling (tuning width) of the neural selectivity. We found that a subset of neurons scaled their spatial selectivity with the size of the object. Our results suggest that neurons in PRR and PMd dynamically adjust their spatial selectivity to the behaviorally relevant spatial space, including spatial parameters of target objects.
\end{abstract}




\section{Introduction}

During visually and memory guided reach movement, reach related areas of the frontoparietal network, parietal reach region (PRR) and dorsal premotor cortex (PMd), transform sensory information into motor plans (Cui and Andersen, 2011). Neurons in these areas are selective for the spatial location of visual cues and the reach goal (Hwang and Andersen, 2012; Gail and Andersen, 2006; Gail and Andersen, 2006; Snyder et al., 1997; Gail et al., 2009; Westendorff et al., 2010). A large body of literature has investigated how and in what reference frames such task related spatial parameters are encoded. Many studies showed eye-centered (Batista et al., 1999; Batista et al., 2007; Buneo et al., 2002; Cohen and Andersen, 2002; Marzocchi et al., 2008; Chang and Snyder, 2010; Bremner and Andersen, 2014) and hand-centered encoding of the reach goal (Caminiti et al., 1990; Caminiti et al., 1991; Johnson et al., 1996; Bremner and Andersen, 2012) in PRR and PMd. There is also strong evidence that neurons not only use absolute eye-centered and absolute hand-centered but also intermediate reference frames (McGuire and Sabes, 2011; Chang and Snyder, 2012). Pesaran and colleagues (Pesaran et al., 2006) showed that PMd neurons encode relative locations of eye-hand-reach goal. In general, available data suggest that spatial encoding in parietal cortex is more biased towards eyecentered while premotor area is more biased towards hand-centered.

Given the above mentioned studies, it is well established that in simple memory guided reach paradigm, single neurons in these areas, encode spatial location of the visual cues and movement goals in different egocentric reference frames. But in more naturalistic situations reaches are often directed towards objects. For example when you want to reach to pick a ruler which is sitting on the table, you can direct your reach towards the left end or right end or maybe the middle of the ruler and grasp the ruler on that site. In such scenario, at the early stages of processing the visual information and planning the movement (when you decide to which part of the ruler you would direct your reach), initially, the reach goal has to be identified relative to the object, that is, the ruler. This initial encoding of the reach goal is independent of the spatial position of the object. At the later stages of the reach planning, the reach goal also needs to be encoded relative to different parts of the own body of the subject in order to start moving the hand. In object-directed reach planning, spatial features of the object partly determines the relevant space for movement planning. Encoding the reach goal relative to another object, 
provides an object-centered - that is, object-to-object; allocentric-code for the reach plan, which has not been yet investigated in the single cell level in reach planning areas of the frontoparietal network of monkeys.

Behavioral studies on human subjects suggest that brain exploits both egocentric and allocentric information for localization of spatial targets in many tasks (Byrne and Crawford, 2010) and there is growing evidence supporting parallel existence of both spatial reference frames within the dorsal stream (Burgess, 2006; Milner and Goodale, 2008). Accordingly, spatial locations are not purely encoded in egocentric frames of reference in the posterior parietal cortex. Previously, object-centered encoding in human subjects was mainly studied in object-based perceptual judgments, for example see (Galati et al., 2000; Vallar et al., 1999; Zaehle et al., 2007). However, in recent studies more attention was paid to investigating the role of allocentric encodings (Chen et al., 2014; Thaler and Goodale, 2011b; Thaler and Goodale, 2011c; Thaler and Goodale, 2011a) while planning and executing reach movements. Results of such studies suggest that the same frontoparietal network which plans and guides movements during target directed-that is, object-to-self; egocentric_reach task is activated during allocentric reach planning and execution. Compared to the egocentric reach, the allocentric encoding increased the BOLD activity in dorsal PMd and right posterior intera-parital sulcus of human subjects (Thaler and Goodale, 2011a). Although the fMRI studies on human subjects provide evidence that in reach tasks the same brain areas are active during egocentric and allocentric reach planning, due to poor spatial and temporal resolution of the BOLD signal data cannot explain the contribution of single neurons during allocentric reach planning. The BOLD signal represents the activity of a population of neurons. Since recent studies reviled that the BOLD signal and the single unit modulations during cognitive tasks are not always in agreement, e.g., (Maier et al., 2008; Kuang S, 2014), it is not clear whether single neurons of PRR and PMd encode spatial location of the cue and the reach goal in object-centered as well as egocentric reference frames. While not many electrophysiology studies exist that directly compare egocentric and allocentric reference frame in monkeys, there is evidence that neurons in parietal area 7a can encode the spatial location of objects in eye-centered (that is, egocentric) reference frame (Andersen et al., 1985), and relative to other task-relevant objects (Chafee et al., 2007; Crowe et al., 2008). Neurons in the same area are gain-modulated by the position of the subject's body in the surrounding environment, that is, in world-centered reference frame (Snyder et al., 1998). In an attempt to directly test object- 
centered reference frame in a saccade task (Sabes et al., 2002), Sabes and colleagues reported lack of object-centered spatial encoding by single neurons of lateral intera-parietal sulcus (LIP). Through a series of experiments, Olson and colleagues (Moorman and Olson, 2007; Olson and Gettner, 1995; Olson and Gettner, 1999; Olson and Tremblay, 2000; Olson et al., 2000) showed that single neurons in supplementary eye field (SEF) of the frontal cortex were selective for the left and right end of the object towards which the saccade was directed. Results of these studies showed that activity of some neurons were modulated by different object centered saccade targets, however, they did not test whether such neurons also show fine tuning to different target locations on the object or they were selective for two categories of targets. If neurons were tuned to different target locations on the object, the first question would be how do spatial features of the object, shape the spatial tuning of the cell?

Here we addressed object-centered spatial encodings in PRR and PMd, both in the single cell and in the population level, when the monkey was performing an object-based reach task. We designed an object-based reach task (see Experimental paradigm) in which monkey was required to memorize a peripheral cue and plan a movement relative to another object. Our task design allowed us to study and compare the spatial encodings during visual memory encoding of the cue and during movement planning in two separate epochs of the trial, as it is described below. Further we asked how the reference frame and spatial selectivity of neurons is modulated with features of the object. Here we tested modulations with size of the object. Present results suggest a flexible, scalable and dynamic reference frame in both areas which is adjusted to the demands of the behavioral needs.

\section{Materials and methods}

In order to study object-centered reference frame, we designed an object-based reach paradigm. Our object-based reach task required the visual instructional cue and the reach goal to be encoded relative to a task relevant object. We conducted two experiments based on this task. In Exp I, we asked whether neurons in PRR and PMd encode spatial locations in object-centered as well as in egocentric reference frames? We also asked whether object-centered encoding is temporally stable or is it specific to particular stages of reach planning. In Exp II, we asked if the 
object size also determines the spatial scaling (tuning width) of the neural selectivity? One male rhesus monkey was seated in a monkey chair in a dimly lit room in front of a fronto-parallel touchscreen. The monkey was head fixed to the chair and the chair was placed in front of the touchscreen so that monkey's mid-sagittal plane was aligned to the center of the screen. Visual stimuli were presented on an LCD screen (19” ViewSonic VX922; onset latencies corrected; background intensity of $0.16 \mathrm{~cd} / \mathrm{m} 2$ ) mounted behind the touchscreen (IntelliTouch, ELO Systems, CA, USA). The screen was $40-45 \mathrm{~cm}$ apart from the monkey's eyes. Bellow, All conversions from $\mathrm{cm}$ to deg is based on the $40 \mathrm{~cm}$ distance.

\section{Behavioral task}

The exact time line of trials in both experiments was as follows (Fig. 1): Monkey initiated the trials by acquiring central eye fixation (224 Hz CCD camera, ET-49B, Thomas Recording) and hand fixation at the touchscreen. The eye fixation stimulus was a filled red square of $0.5 \mathrm{~cm}\left(0.72^{\circ}\right)$ size and $7 \mathrm{~cd} / \mathrm{m}^{2}$ intensity, and the hand fixation stimulus was a filled white square of $0.5 \mathrm{~cm}\left(0.72^{\circ}\right)$ size and $13 \mathrm{~cd} / \mathrm{m}^{2}$ intensity. The monkey was required to maintain ocular fixation throughout each trial within an invisible window of $2-3 \mathrm{~cm}\left(2.86-2.89^{\circ}\right)$ radius. Manual fixation had to be maintained within an invisible window of $3 \mathrm{~cm}\left(2.89^{\circ}\right)$ radius until the fixation stimulus disappeared (GO cue). In the case of unsuccessful fixation, the trial was aborted and repeated at a random later time during the experiment. After valid eye and hand fixation of random length 600-1100 ms, an array of five boxes, horizontally connected with a line, appeared for $400 \mathrm{~ms}$ (see Figure 1, Reference object). The position of the boxes indicated the potential positions of the pending cue. Monkey was required to keep these positions in mind for proper task performance, as it is described below. Following the offset of the reference object a visual cue was presented for $340 \mathrm{~ms}$. The cue consisted of a small dot of $0.27 \mathrm{~cm}\left(0.39^{\circ}\right)$ diameter at the randomly chosen position of one of the five reference object boxes, indicating the target box to which the monkey should later reach. Inter-stimulus interval (ISI) between reference object and the subsequent cue was 80 ms. Cue was succeeded by a variable memory period 700-1200 ms. After the memory period, a decision object was presented to the monkey. In Exp I, the decision object was graphically identical to the reference object and in Exp II in some trials it was up-scaled in size, as will be explained below.

The reference and decision objects were spatially congruent in location only in $50 \%$ of trials (see below). Appearance of the decision object was succeeded by a delay period of $646 \mathrm{~ms}$ during 
which time the decision object was available on the screen and monkey was required to maintain eye and hand fixation and not initiate the movement. After the delay period, the hand fixation stimulus disappeared. This served as the GO cue, indicating to the monkey to start the reach movement. He had to touch the target location on the screen within $1000 \mathrm{~ms}$ after the GO cue while holding central ocular fixation. The monkey was required to hold the target for $400 \mathrm{~ms}$ to count the trial as 'successful'. After a successful trial, monkey received a visual and an acoustic feedback and a drop of juice as reward. The visual feedback was a small filled light gray circle of $0.27 \mathrm{~cm}\left(0.39^{\circ}\right)$ diameter on the target box of the decision object and the acoustic feedback was a high-pitched tone. Reference object, decision object and the cue had low intensity gray tone (2 $\left.\mathrm{cd} / \mathrm{m}^{2}\right)$.

The cue appeared at one of the five reference object positions. Monkey was instructed to hit one of the five decision object boxes which corresponded to the reference object box at which he had seen the cue, e.g., for a cue seen at reference object box \#2 monkey should reach to decision object box \#2, irrespective of the absolute position of the decision object. If the reach endpoint was within an invisible elliptical window (semi-minor axis $1.2 \mathrm{~cm}\left(1.72^{\circ}\right)$, horizontal; semimajor axis $4 \mathrm{~cm}\left(5.71^{\circ}\right)$, vertical) from the target box the trial was counted successful. Throughout the text, for each trial of the task the term 'cue' refers to the dot stimulus presented before the memory period (Figure 1, Spatial cue) and 'target' refers to the box of the decision object that corresponds to the cued box of the reference object.

Detailed specifications of the stimuli which were different for the two experiments are as follows:

Exp I: In Exp I, boxes of the reference and decision object were $0.35 \mathrm{~cm}\left(0.50^{\circ}\right)$ squares, and centered $2.8 \mathrm{~cm}\left(4.00^{\circ}\right)$ apart from each other (see Figure 2a).

Exp II: In Exp II, in half of the trials, the reference object was the same size as in Exp I (long object trials) and in other half it was half the length but otherwise identical (short object trials, see Figure 2b). In short object trials, boxes on the reference object were $1.4 \mathrm{~cm}\left(2.00^{\circ}\right)$ apart. The decision object was always (in both long and short trials) identical to the long reference object. 


\section{Spatial configuration of the stimuli}

Figure 2 illustrates spatial configuration of the stimuli in Exp I and II. Monkey's body midsagittal plane, eye and hand fixation points were all aligned to the center of the screen. Therefore any location and displacement of the stimuli/reach goal on the screen had equal value in all above-mentioned egocentric reference frames. Reference object was randomly presented at the left or right side, relative to the center of the display (two different egocentric locations; see Figure 2a). Presentation of the reference object at each location was succeeded by presentation of the decision object, randomly to the left or right relative to the center of the screen. Here and throughout the text, location of the object refers to the position of the center of mass of the object on the screen, i.e., the center point of the central box on the object.

Exp I: In Exp I, reference and decision object had the same left and right egocentric locations $\left((x, y)=( \pm 1.4,+2.65) \mathrm{cm}=( \pm 2,3.79)^{\circ}\right)$. Location of the reference (decision) object boxes on the screen - -i.e., egocentric position of the boxes and the cue (target) — when the object presented at the left side partly overlapped with those when the object presented at the right side. When the object was at the left side, box \#2 to \#5 (starting from the leftmost box on the object) had the same locations on the screen (same egocentric positions) as box \#1 to \#4, respectively, when the object was presented at the right side (see Figure 2a-Potential egocentric locations of cue and reach goal). Therefore, while the cue and the reach target could take five different positions relative to the reference and decision object, respectively (five different object-centered positions) in total, they had six different egocentric locations on the screen. The 20 different

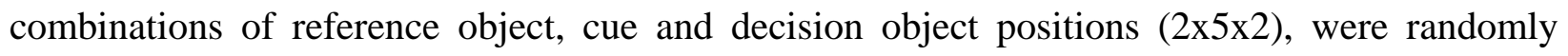
presented. With this constellation of stimuli, the cue and the reach goal always had the same object-centered position (relative to the reference and decision object, respectively). Whereas, only in half of the trials when the reference and decision objects were spatially congruent (both presented either at the left side or at the right side), the cue and the reach goal had the same egocentric locations. Incongruency of the cue and the reach goal in other half of the trials mandate object-based encoding. Since occurrence of the incongruent trials was unpredictable to the monkey, the only way to successfully perform the task was to follow object-based encoding of the cue and the reach goal in all trials. 
Exp II: In Exp II, both long and short reference objects were presented in the left and right locations as in experiment one. Unlike Exp I, location of the decision object was always incongruent to the reference object $\left((x, y)=( \pm 2.35,4.41) \mathrm{cm}=( \pm 3.36,6.29)^{\circ}\right)$. As in Exp I, the cue and the reach goal always has the same object-centered position (relative to the reference and decision object, respectively). Figure 2b shows how the locations of the reference object boxes overlap (i.e., have the same egocentric locations) in different conditions: Long left and long right reference objects: boxes \#2 to \#5 with \#1 to \#4; Short left and short right reference objects: boxes \#3 to \#5 with \#1 to \#3; Long left (right) and short left (right) reference objects: boxes \#2, $\# 3$ and \#4 with \#1, \#3 and \#5. Long object and short object trials were presented in alternating blocks. In the long object block of the trials, all 20 different combinations of reference object, cue and decision object positions ( $2 \times 5 \times 2)$ were randomly presented. In the short object block, in first 10 trials with reference object left condition and in the second 10 trials reference object right conditions were presented. In each sub block, 5 cue location and 2 incongruent decision object location where randomly interleaved.

\section{Animal preparation and neural recordings}

The procedures for animal preparation and neural recordings were described previously (Westendorff et al. 2010) and are here repeated for completeness. Only the numerical values have been adjusted to the current experiment. "One male rhesus monkey (Macaca mulatta; monkey K) was trained to perform the object-based reach task. The monkey was implanted with a titanium head holder and two magnetic resonance imaging (MRI)-compatible recording chambers, custom-fit to the monkeys' heads (3di and Thomas Recording). Chamber positioning above PRR [Horsley Clarke coordinates: (10 mm, contralateral; $13 \mathrm{~mm}$ posterior), and PMd (18.5 mm contralateral; $20 \mathrm{~mm}$ anterior) was guided by presurgical structural MRI and confirmed by postsurgical MRI. Sustained direction-selective neural responses during center-out reach planning (memory period) were used as a physiological signature in both areas to confirm the region of interest. Both chambers were implanted contralaterally to the handedness of the monkey (left hemisphere). All surgical and imaging procedures were conducted under general anesthesia. We used two five-channel microdrives ("mini-matrix"; Thomas Recording) for extracellular recordings, mostly simultaneously in both chambers. The raw signals of the electrodes were pre-amplified (20x; Thomas Recording), band pass filtered, and amplified (154 
$\mathrm{Hz}$ to $8.8 \mathrm{kHz}$; 400-800x; Plexon) before online spike sorting was conducted (Sort Client; Plexon). Spike times and spike wave forms were recorded and later subjected to additional offline sorting (Offline Sorter; Plexon).”

\section{Visual memory and motor goal encoding}

We analyzed the mean firing rate of the neurons during visual memory encoding of the cue (50350 ms window after the cue offset) and movement planning (350-50 ms window preceding the GO cue). For time resolved analysis, different time windows are used which is specified bellow.

In order to distinguish object-centered and egocentric reference frames during visual memory period, for each neuron we assessed spatial tuning of the response to the five cue stimulus positions in trials when the reference object was presented at the left side (reference-object-left tuning curve for Exp I; short- and long-reference-object-left tuning curves were analyzed separately in Exp II, see below) with the trials when the object was presented at the right side (reference-object-right tuning curve for Exp I; short- and long-reference-object-right tuning curve for Exp II). During movement planning we compared the spatial tuning of the response to the five target positions in trials when the decision object presented at the left side (decisionobject-left tuning curve for Exp I) with the trials when the decision object presented at the right side (decision-object-right tuning curve for Exp I). In Exp II, we only analyzed the visual memory period as explained below.

\section{Neuron selection}

In Exp I, we included neurons with significant tuning for both reference-object-left and -right tuning curves (non-parametric one-way ANOVA, Kruskal-Wallis, $\alpha=0.05$, unless mentioned otherwise) to analyze visual memory period. Cells with significant tuning for both decisionobject-left and -right tuning curves were included to analyze movement planning period. In temporal analysis of reference frame (see below), we required neurons to be tuned: for the reference-object-left and -right condition in time intervals $280-430 \mathrm{~ms}$ or $330-480 \mathrm{~ms}$ after the cue onset, to be included in the analysis of the visual encoding period; for the decision-object-left and -right condition in time intervals $350-500 \mathrm{~ms}$ or $400-550 \mathrm{~ms}$ after the onset of the decision object, to be included in the analysis of the movement planning period. 
In Exp II, we only analyzed spatial encodings during visual memory period. We required neurons to be significantly tuned for at least three out of four reference object conditions (i.e., long-reference-object-left, long-reference-object-right, short-reference-object-left, and shortreference-object-right). Included neurons had at least 5 repetitions per task condition.

\section{Object-centered vs egocentric hypothesis}

Figure 3a illustrates reference-object-left and reference-object-right tuning curves of three ideal cells for Exp I, illustrating object-centered, egocentric and intermediate (mixed egocentric and object-centered) reference frame. If a cell is encoding the location of the cue in object-centered reference frame then selectivity of the neuron depends only on the relative position of the cue and the reference object. Therefore, if an ideal neuron shows a certain pattern of tuning to different cued locations on the reference object when the object is on the left side, it has to retain its tuning pattern, in the trials that the object is presented at the right side. This implies that the neuron is encoding the cue location in the object coordinate. Whereas when an ideal neuron is encoding the cue in egocentric reference frame, the selectivity depends only on the egocentric location of the cue (i.e., location of the cue on the screen) regardless of absolute position of the preceding reference object. In this case, if one compares the reference-object-left and referenceobject-right tuning curves, only for cue positions with equal egocentric locations the neuron would demonstrate the same activity (see Spatial configuration of the stimuli and Figure 2a). The same logic holds true for the target and decision object.

Given the present spatial configuration of the stimuli, when the reference object relocates from one side of the screen to the other side, an object-centered neuron should also relocates its spatial tuning curve to the cue, from one side to the other. Thus if the two tuning curves were plotted in the screen coordinate (Figure 3a), a shift of the tuning curve from left to right as large as the object's shift on the screen $X$ axis would be expected from an object-center neuron while the shape of the selectivity of the neuron to different location on the object has to be maintained the same. The same logic holds true for spatial tuning curves to the motor goal.

\section{Signal to noise ratio}


In order to quantify the strength of the tuning, for each tuning curve we took the $F$ statistics of ANOVA as a measure. The mean of this measure for reference-object-left and -right tuning curves and for decision-object-left and -right tuning curves were used to evaluate how strongly each individual neuron was tuned.

\section{Quantifying the reference frame}

We characterized the reference frame of each individual cell with two different methods, a nonparametric and a parametric approach.

Correlation analysis, tuning curve similarity: As explained above, object-centered and egocentric hypotheses predict fully and partly similar object-left and object-right tuning curves, respectively. Accordingly, for each cell we took the similarity of the two tuning curves as a measure to quantify to what extend each hypothesis can explain the response of the neuron. As a measure of similarity, we used the Pearson's linear correlation coefficient (similar to MulletteGillman et al., 2005) between each pair of the tuning curves-i.e., reference-object-left and right; decision-object-left and -right. The higher the correlation coefficient, the more similar the shape of the two tuning curves. Here and throughout, for all analysis which involved correlation coefficients, we applied Fisher transformation to all the correlation coefficients so that we can use student's ttest to compare correlation values across neurons and task conditions. Also for all correlation based analyses, in order to account for variability in the firing rate, tuning curves were re-sampled 100 times using the Jackknife method (Efron, ) and the correlation coefficient for each pair of the re-sampled tuning curves was quantified. Re-sampling the tuning curves provided a distribution for each correlation coefficient. The mean of the distribution was taken as the similarity between the two curves and was used for further analysis. In this analysis we quantified the similarity between the shape of the tuning curves as described above and we

discarded possible gain modulations across the tuning curves due to the limitations of the correlation method.

For each pair of the tuning curves, we quantified two correlation coefficient based on two hypotheses, as follows:

Object-centered correlation: correlation coefficient between the points with the same objectcentered locations. (see Spatial configuration of the stimuli and Figure 3a). 
Egocentric correlation: correlation coefficient between the points with the same egocentric locations. (see Spatial configuration of the stimuli and Figure 3a).

Hypothesis I, object-centered reference frame: According to this hypothesis the neuron is encoding the position of the cue stimulus or the reach goal relative to the object. This hypothesis predicts object-centered correlation is larger than the egocentric correlation for object-centered encoding.

Hypothesis II, egocentric reference frame: According to this hypothesis the neuron is encoding the position of the cue stimulus or the reach goal in egocentric reference frame, and thus this hypothesis predicts egocentric correlation is larger than the object-centered correlation for egocentric encoding.

Each of the above-mentioned correlation values provides a measure that determines the validity of the corresponding hypothesis for each cell. The difference between the two correlation values provides a continuous measure which points out the neuron's dominant reference frame. In the result part this measure will be marked by objCorr-egoCorr. Positive values indicate that the tuning profile of the neuron was better explained by the object-centered hypothesis.

Parametric modeling, tuning curve shift: One of the predictions of the object-centered hypothesis, is the shift of the tuning curve from left side to the right side along with the shift of the object, while preserving the shape. On the other hand egocentric hypothesis predicts no shift for the tuning curve for the points where left-object and right-object boxes overlap. In order to specifically measure the shift, we fitted a parametric model to the tuning curves as was commonly used in previous reference frame studies in parietal and motor cortical areas (Chang and Snyder, 2010; Chang and Snyder, 2012; McGuire and Sabes, 2011).

We fit each of the two tuning curves (the object-left and object-right tuning curves) to a fourparameter cosine function. The functions were simultaneously fitted to the sampled responses in every trial.

$$
\begin{array}{ll}
R_{\text {object-left }}=A_{L} \operatorname{Cos}\left(2 \pi f x+\varphi_{L}\right)+M_{L} & \text { Eq } 1 \\
R_{\text {object-right }}=A_{R} \operatorname{Cos}\left(2 \pi f x+\varphi_{R}\right)+M_{R} & \text { Eq } 2
\end{array}
$$


Where $R$ is the firing rate in a given trial; $x$ (defined in $\mathrm{cm}$ ) is the laterality of the cued or target box relative to the center of the reference or decision object, respectively; $A_{x}$ and $M_{x}$ determine the peak amplitude and mean of the tuning curves; parameter $f$ which determines the width of the tuning curves was assumed to have a common value across the two curves, assuming that the width of the tuning is not modulated by changing the object's location on the screen. The model was fitted to the firing rate using nonlinear least square optimization (we used nlinfit function in Matlab and applied robust fitting). We chose a cosine model based on the shape of the response profiles to different locations on the object that we observed in our data. During the fitting, parameters were constrained as follows: $0<\beta_{1} ; 0 \leq \beta_{2} \leq 2 ;-0.5 \leq \beta_{3} \leq 0.5 ; 0<\beta_{5}$ and $-0.5 \leq \beta_{6} \leq 0.5$.

For each neuron we fit this model to the mean firing rate once during visual memory period (Eq 1 and 2 to the reference-object-left and -right trials, respectively) and once during motor goal planning period (Eq 1 and 2 to the decision-object-left and -right trials, respectively). Since we assumed a cosine tuning profile with equal width across the two object locations, the difference between the phases of the two cosine functions determines how much the spatial selectivity of the neuron shifts relative to the center of the object when the absolute location of the object shifts from left to right. Therefore, we defined an index propShift-that is, proportion of shift-which serves as a continuous measure of reference frame.

$$
\text { propShift }=\frac{\varphi_{R}-\varphi_{L}}{2 \pi f}
$$

propShift $=0$ indicates that the tuning curve did not shift when the object shifted from left side to the right, indicating a pure egocentric reference frame whereas propShift=1 indicates a shift of the tuning curve as large as the shift of the object on the screen, which indicates an objectcentered encoding. Values between 0 and 1 , reflects partial shift of the tuning curves which is an indication for intermediate reference frames.

For this analysis, we included those neurons which their tuning profile could be fit to the model. When comparing the distribution of the tuning shifts across the areas, we selected those units for which the estimated parameters were converged within the predefined boundaries, as mentioned above. As was explained before, for each neuron we fit this model once during visual memory period and once during motor goal planning period. For paired comparison of visual v.s. motor 
goal tuning of individual cells, in order to increase the statistical power we included all the cells which were tuned during motor goal and visual memory encoding.

\section{Scaling of the spatial tuning to the object size}

Figure 3b illustrates responses of two ideal cells during visual memory period for Exp II, one representing scaled tuning curve to the size of the object and one representing non-scaled tuning curve. If the tuning curve is scaled with the size of the object, the pattern of selectivity for different locations on the object should be preserved when the size of the object changes. The width of the tuning curve scales to the size of the object, therefore, the neuron will represent size-invariant object-centered tuning.

To quantify scaling of the tuning, for each neuron we used the Pearson's linear correlation coefficient between the tuning curves in long-object and short-object trials based on two following hypotheses:

CorrScaled: The correlation coefficient between response of the neuron when cue was on box \#1 to \#5 of long object respectively with response to cue on box \#1 to \#5 of the short object (see Figure 2b) separately, when the long and short objects were presented to the left and when they were presented to the right. For each cell, we took the mean of the CorrScaled for object-left and -right tuning curves for further analysis.

CorrNonScaled: The correlation coefficient of response between the corresponding overlapping points-i.e., correlation between response when cue was on box \#2, \#3 and \#4 of the long object respectively with response to cue on box \#1, \#3 and \#5 of the short object; see Figure 2b. We took the mean of the correlation coefficient across object-left and object-right trials for further analysis.

Hypothesis I, scaled tuning curve: according to this hypothesis the pattern of selectivity to different locations on the object is retained across short and long object trials, with possible gain modulation. Thus, this hypothesis predicts high similarity between the tuning curves in longobject and short-object trials (in Exp II we only analyzed the visual memory period). Therefore hypothesis I predicts that CorrScaled is larger than CorrNonScaled. It is important to note that 
hypothesis I rests on two underlying assumptions: the cell is object-centered and the tuning curve scales relative to the center of the object.

Hypothesis II, subsampled tuning curve: according to this hypothesis the tuning profile is not scaled to the size of the object. Instead, in short-object trials tuning profile is sampled with higher spatial resolution. Thus, hypothesis II predicts similar response for the three overlapping cue locations between the long and short objects (see Spatial configuration of the stimuli). Therefore hypothesis I predicts that CorrNonScaled is larger than CorrScaled.

\section{Results}

In experiment I we recorded 82 single neurons in PRR and 99 in PMd; in experiment II we recorded 45 neurons in PRR and 44 in PMd, from monkey $\mathrm{K}$ while he was performing objectbased reach task. The monkey performed the task with $72 \%$ and $63 \%$ averaged success rate in Exp I and II, respectively.

\section{Result of Exp I:}

We asked whether neurons in PRR and PMd used object-centered reference frames when the monkey was required to memorize a peripheral visual cue and to plan a reach relative to other objects. We found many neurons in both areas that encode the position of the visual cue and the movement goal in an object-centered reference frame (relative to the reference-object and decision-object, respectively), but also neurons with egocentric or intermediate reference frames.

Examples of single neuron responses: During visual memory period, many neurons in both areas encode the location of the cue in object-centered reference frame- that is, cue relative to the reference object. Figure 4a illustrates the reference-object-left and -right tuning curves of three example neurons. Pattern of selectivity of object-centered neurons for different locations on the reference object was independent of absolute location of the object on the screen (left panel). When the reference object shifts from the left to right side of the screen, the spatial selectivity of the neurons also shifts from the left side to the right side while conserving the same relative selectivity for different locations on the reference object. A different subset of neurons encode 
the egocentric location of the cue, Figure 4a center panel exemplifies such units. Egocentric neurons showed same selectivity when overlapping boxes across two object locations were cued but different activity for non-overlapping boxes.

During movement planning we also observed that some neurons encode the reach goal in objectcentered reference frame (that is, reach goal relative to the decision object) and some in egocentric reference frame. Figure 4b exemplifies neurons with object-centered and egocentric encoding of reach goal. It also became evident that neurons in the two areas do not belong only to the two ends of the object-centered and egocentric spectrum, but rather cover a spectrum of object-centered to egocentric reference frames. The intermediate egocentric-object reference frames can be indicated by partial shift of the object-left to object-right tuning curves. Figure 4a illustrates an example of an intermediate reference frame.

Correlation analysis, tuning similarity: In order to quantify the reference frame, the egocentric and object-centered correlation coefficients were calculated for each neuron (see Materials and methods). The objCorr and egoCorr values for example cells in Figure 4 are as follows (before applying the Fisher transformation): (objCorr,egoCorr) for visual memory object-centered (0.01,0.98); visual memory egocentric $(0.99,0.85)$; visual memory intermediate $(0.71,0.67)$; motor goal object-centered $(0.69,0.96)$; motor goal egocentric $(0.94,0.82)$. Figure 5 illustrates the objCorr-egoCorr distribution for the PRR and PMd neurons, during visual memory encoding and motor planning (see Materials and Methods for time interval of analysis). As the figure shows, during visual memory encoding majority of neurons in PRR were encoding the object-centered location of the cue (qualified by significant shift of the distribution towards the positive values; sign test: $\mathrm{p}<0.01$ ) where as in PMd both object-centered and egocentric encoding were equally present (qualified by no significant shift of the distribution from zero; sign test: $\mathrm{p}>0.65$ ). During the movement planning period, majority of neurons in both areas encode the reach goal in egocentric reference frame (qualified by significant shift of the distributions to the left, sign test: PRR $\left.\mathrm{p}<2.8 \times 10^{-4} ; \mathrm{PMd} \mathrm{p}<2.3 \times 10^{-8}\right)$.

Parametric modeling, shift analysis: In order to measure the shift of the tuning curves when the location of the object shifts on the screen, a parametric model was fitted to the tuning curves (see Materials and Methods), separately during visual memory encoding and movement planning. Neurons which fulfilled our goodness-of-fit criterion were included in subsequent analysis. 
Figure 6a shows distribution of the shift values for PRR and PMd neurons during visual encoding movement planning. Shift of the distribution towards value one, indicates shift of the tuning curves as large as the shift of the object on the screen. In both areas, the distribution of shift values is closer to one during visual memory period as compared to the movement planning period. The tuning shift across the areas was compared separately during visual memory and motor goal encoding. Although the statistical test did not reach the significance level (Kolmogorov-Smirnov test, $\alpha=0.05$ ), results show a non-significant trend that PRR is more object-centered than PMd (PRR>PMd: visual memory $\mathrm{p}>0.2$; motor goal encoding $\mathrm{p}>0.4$ ) both during visual memory and motor goal encoding. The fact that statistical test did not reach significance might be due to insufficient statistical power. For those neurons which had a good fit for both visual memory and movement planning, we did a paired comparison of the shift value between the visual memory and movement planning. Data showed a significant shift of the tuning in the direction of the object shift during visual memory encoding compared to the movement planning period (PRR sign test: $\mathrm{p}<0.002$, paired t-test: $\mathrm{p}<0.008$; $\mathrm{PMd}$ sign test: $\mathrm{p}<0.02$, paired t-test: $\mathrm{p}<0.0006$; see Figure 6b). Result of this analysis indicates the dominance of object-centered encoding during visual memory encoding compared to the movement planning.

For tuning similarity analysis, 34 of PRR and 79 of PMd cells were included in visual memory period; 42 of PRR and 82 of PMd cells were included in movement planning period. For measuring the tuning shift, based on the goodness of fit criteria (see Materials and methods), visual tuning of 20 PRR and 47 PMd neurons, and motor goal tuning of 28 PRR and 41 PMd neurons were analyzed when comparing tuning shift across the areas; 21 PRR and 39 PMd cells were analyzed in paired comparison of visual v.s. motor goal tuning of individual cells.

Dynamic reference frame: In order to see how the two types of reference frames evolve over time, we conducted a time-resolved correlation analysis. We looked at the egocentric and objectcentered correlation coefficients within time intervals of $150 \mathrm{~ms}$ sliding in $50 \mathrm{~ms}$ steps. Based on our selection criteria (see Neuron selection), 36 of PRR and 75 of PMd cells were included in visual period; 43 of PRR and 76 of PMd cells were included in movement planning period. Figure 7a shows mean egocentric and object-centered correlations across the subpopulation of the tuned neurons (see Materials and methods for unit selection criteria); the values are plotted at the midpoint of the time window and error bars indicate standard error of the mean; time 
intervals with significant difference between egocentric and object-centered correlations are highlighted by '*' (paired t-test, $\alpha=0.05$ ). We performed this analysis once for visual memory encoding where data was aligned to the cue onset and once for movement planning where data was aligned to the onset of the decision object. In order to analyze the reference frame during the transition phase from visual memory to movement planning, for time intervals before the decision object onset the reference-object tunings were used-i.e., tuning for the cue relative to the reference object at late memory interval. For the time intervals after and including the decision object onset event, we used decision-object tunings-i.e., tuning for the reach goal relative to the decision object; this time interval is indicated by red shaded area in Figure 7.

From this analysis it is clear that on average the visual memory encoding is more object-centered compared to movement planning but reference frames evolve with different dynamics in the two areas. In PMd, majority of neurons start with egocentric encoding of the cue location; later at early visual memory encoding there is a balanced representation of the two reference frames which changes to dominant object-centered encoding at the late visual memory period. Whereas, most of the PRR neurons start with object-centered encoding of the cue and maintain this reference frame through the memory period. While in both areas object-centered encoding of the cue is dominant at late memory period, after onset of the decision object (which indicates the time point when the monkey was provided with enough information to start planning the movement) the object-centered encoding of the cue gradually changes to egocentric encoding of the reach goal.

Signal to noise ratio: From Figure 7a it is apparent that after the cue onset, reference frames emerge faster in PMd than in PRR. This could happen due to a faster and stronger tuning of PMd neurons. In order to test this hypothesis, we quantified the strength of the selectivity for all individual neurons. For each cell, we looked at the F statistics of ANOVA separately for objectleft and object-right tuning curve in different time intervals (sliding window as explained in section Correlation analysis, dynamic reference frame). We used the mean of the two selectivity values as the tuning strength of a given neuron. Figure 7b illustrates mean tuning strength across the population of all neurons. In the first 100-200 ms after the cue onset PMd shows faster and stronger tuning than PRR. Performing the same analysis on the sub-population of visual memory 
tuned neurons and movement planning tuned neurons resulted in the same conclusion (see Materials and methods for unit selection criteria).

\section{Result of Exp II}

In Exp II, we recorded 68 single neurons in PRR and 53 in PMd; 41 of PRR and 44 of PMd cells were included in visual memory encoding analysis (see Material and Methods for criteria to select neurons).

Size scaling of tuning curves, example unit response: We found that many neurons in both areas scaled their spatial selectivity with the size of the object. Such neurons retain their pattern of selectivity to different locations on the object, independently of the size and absolute location of the object. Some of these neurons were gain modulated with the size and location of the reference object. Figure 8a illustrates the normalized tuning curves of an example cell from PMd for short and long reference objects, separately when the objects were presented at the left and when they were presented at the right. The dotted curve represents the predicted response of the cell for short object trials based on the tuning curve in long object trials, according to the scaled tuning curve hypothesis (see Materials and Method), which predicts the width of the tuning curve scales with the size of the object . As the figure shows, the short-object tuning perfectly fits the prediction of the hypothesis, both for object at the left and at the right.

Reference frame in short-object and long-object trials: We first compared the reference frame of individual cells during the visual memory period between short-object and long-object trials. We evaluated the reference frame using the correlation technique (see Materials and methods). The slope of the regression line for both areas is positive but did not reach significance (slope of the regression line and the 95\% confidence interval for PRR was $0.01 \pm 0.33$ and for PMd was $0.25 \pm 0.29)$.

Size scaling of tuning curves, population: In the example tuning curve of Figure 8a, the neuron is encoding the cue location in an object-centered reference frame. However, in general, a neuron can show totally different tuning shapes for the two reference-object locations, yet when the object scales at each location, the related tuning curve will scale too. Therefore, independently of the reference frame of the neurons, we tested if individual neurons show size invariant tuning to the cue locations on the object, separately for reference-object-left and -right conditions. The size 
invariant tuning hypothesis requires the tuning curves to be scaled relative to the center of the object. We compared the shape of the tuning curves between the long-object and short-object conditions using the correlation method (see Materials and methods). Figure 8b illustrates the distribution of the CorrScaled-CorrNonScaled (see Materials and methods) across the population of neurons in both areas. Result shows that different neurons scale their tuning curves to different degrees so that in the population of neurons in each area, the full spectrum from fully-scaled to non-scaled can be observed. Applying a sign-test on each population separately did not result in a significant difference of the median from zero in either population (PRR: p>0.34; PMd: $\mathrm{p}>0.09)$.

\section{Discussion}

We showed that individual cells in PRR and PMd encode object-centered location of the visual cue and motor goal during object-based reach task. We also showed that when the size of the object changes, a subset of cells in each area scale the width of their tuning with the object size so that they represent size-invariant selectivity to different locations on the object, independently of the size and absolute position of the object. During the transition period from visual memory encoding to movement planning, both PRR and PMd change their preferred reference frame from object-centered to egocentric so that in both areas, encoding of the visual memory period is more object-centered and encoding of the movement plan is more egocentric.

\section{Comparison with other electrophysiology studies}

Although there are not many studies directly comparing egocentric and allocentric reference frame at the level of single cell in monkeys, there is evidence that neurons in parietal area 7a can encode the spatial location of objects in an eye-centered (i.e., egocentric) reference frame (Andersen et al., 1985) as well as relative to other task-relevant objects (Chafee et al., 2007; Crowe et al., 2008). Neurons in the same area are gain-modulated by the position of the subject's body in the surrounding environment - that is, in world-centered reference frame; (Snyder et al., 1998). Regarding the reference frames which are utilized for reach movement planning, most of the electrophysiology studies are focused on various types of putative egocentric references (e.g. 
eye-centered and hand-centered, see Introduction) which could be used during reach movement preparation.

Sabes and colleagues trained a monkey to perform object-based saccade (Sabes et al., 2002). Although about $10 \%$ of the cells they analyzed, showed significant object-centered effects, compared to the overall modulation of the neural firing rate the effect was so small that they concluded lack of object-centered encoding during object-based saccade in area LIP. Areas LIP and MIP (PRR) are seen as two equivalent functional units for the oculomotor and the skeletomotor systems, respectively. The previously observed lack of object-centered coding of saccade plans could be either due to the difference between the oculomotor and skeletomotor systems, or due to the fact that small differences in task design allowed different cognitive strategies. Although object-based saccade behavioral paradigm and the object-based reach task in the present work share many similarities, there are subtle differences which we believe are important to control the strategy that the monkey follows to perform the task. In the previous study, as was claimed by the authors one of their main concerns was to prevent the monkey from using local features of the object outline around the target location rather than using objectcentered location of the target. Authors approached this issue by choosing especial shape for the object (object constructed of four fingers and one elongated handle). However, presence of the spatial cue on top of the object (simultaneous presentation of the cue and the object), caused the overall shape of the presented object to be different for different object centered locations of the cue (when different fingers on the object were cued). Monkey could advantage from this information during movement planning and try to reconstruct a previously observed patter on the object instead of comparing the location of the cue with it. Assuming that the above mentioned issue was true, in order to overcome this problem, in the present task design we introduced a short time gap between the reference object and the cue (Figure 1) so that the cue is not presented simultaneously with the object. This will encourage the monkey to compare the location of the cue with the memorized location of the reference object. Thereby we avoid encoding of different visual patterns instead of visual positions that could be used as alternative strategy to actually comparing the location of the two stimuli.

In a series of experiments (Moorman and Olson, 2007; Olson and Gettner, 1999; Olson and Tremblay, 2000; Olson and Gettner, 1995) Olson and colleagues studied object-centered 
encoding in area SEF of the frontal cortex. They showed that when the monkey was required to make a saccade to either ends of an elongated bar object, cells showed differential firing rate for left and right ends of the object independently of the absolute location of the object. They also showed cells were selective for left or right target when monkey had to choose the saccade goal from an array of two discrete points independently of the absolute location of the array. Data in those studies provides evidence for selectivity of the neural responses to two classes of targets (left and right target). However, with the binary encoding of left and right it might not really be possible to decide on the reference frame in many cases. It is also not clear to what extend object-centered selectivity is maintained when other locations on the object is the target of saccade. Data does not explain whether it was a categorical selectivity or neurons can be finely tuned to different target location on the object, similar to the tunings which were reported in the present study for PRR and PMd cells. In this respect, our result adds to the previous findings by showing that neurons in the reach network of parietal and frontal cortices show selectivity to different locations on objects. We found object-centered encoding not only in the frontal cortex, but also in the parietal cortex where it is often assumed to be involved in egocentric encoding of movement parameters. The type of object-centered encoding that we found goes beyond a categorical selectivity to two classes of targets. Neurons in both PRR and PMd become selective for different locations on the object, when the upcoming reach will be directed to that site of the object. They also adjust their selectivity according to the variable features of the object, so that their pattern of spatial selectivity relative to the object is invariant.

\section{Dynamic reference frame}

If the predominant spatial encoding in PPC depends on the behavioral constraints of the task then we expect that this predominance might change over the course of the trials. This is because the behaviorally relevant spatial task parameters change during the trial. At the early stages of a trial, instructional stimuli are more relevant whereas at the later stages physical parameters of the reach become relevant. Only very few studies investigated temporal evolution of reference frames in the parietal cortex. Buneo and colleagues (Buneo et al., 2008) showed that during instructed delayed reach task, reference frame which was used by area 5 and PRR to encode the cue location and reach goal remained constant after reach target onset, both at the single cell and population level. However, another study (McGuire and Sabes, 2011) reported slight shift 
towards more eye-centered encoding as compared to the hand-centered reference frame, at the later epoch of the behavioral trial (closer to the time of the movement initiation) in the same two areas. Additionally, a more recent study (Bremner and Andersen, 2014) showed that neurons in area $5 \mathrm{~d}$ switched their encoding from hand-relative-to-direction of gaze to target-relative-to-hand when subjects received enough information that allowed them to start planning the movement. Although these studies were focused on various egocentric references which are believed to be utilized by parietal neurons, their findings suggest that subregions of the posterior parietal cortex can flexibly encode task relevant spatial relations in different epochs of the behavioral task. Along this line, our data clearly shows transformation from object centered during late memory period (late phase of the first memory period, before the presentation of decision object) to egocentric during movement planning period within the population of both PMd and PRR. Previously, evolution of a particular encoding over the course of a trial within an area was taken as evidence that the transformation to this reference frame was occurring within the same area (Buneo et al., 2008). Additionally, as was suggested by mathematical models (Avillac et al., 2005; Zipser and Andersen, 1988; Pouget and Snyder, 2000; Pouget et al., 2002), neurons which encode spatial information in intermediate reference frames play an important role in transforming information across different frames of references. In our data, presence of intermediate reference frame (intermediate between egocentric and object-centered, qualified by partial shift of the tuning curves) as well as evolution of egocentric reference within a population of neurons which were encoding object-centered reference frame, provides further evidence in support of the hypothesis that transformation between egocentric and object-centered references, at least partly, occurred within PRR and PMd areas. However in a strict sense we cannot rule out the alternative that PRR and PMd inherit this information from other areas.

As the present data shows, while during memory period both areas represent the motor goal in an abstract object-centered format, immediately after presentation of the decision object (the location of which is the only missing piece of information to plan the final movement) both areas start to gradually shift to egocentric encoding of the motor goal. In our task design the decision object stays on for a few hundred milliseconds during which the monkey has to maintain fixation and withhold the movement. Therefore, alternatively to immediate evolution of the egocentric encoding, transition from object-centered to egocentric encoding could occur at the late stage of the delay period and at the time of the GO signal or shortly before that. Given the fixed length of 
the delay period from decision object onset till the GO signal, the time of the GO signal is predictable to the monkey which makes the latter a plausible scenario. However, data suggests that egocentric encoding of the motor goal starts immediately after the time when enough information is provided to make the transformation possible. This result is comparable with the prediction from behavioral studies (Chen et al., 2011). While object-centered encoding provides a more robust representation of the space, such early transformation has the benefit of preparing the system for fast reactions (Chen et al., 2011). Present task was not designed to properly study reaction times therefore the question that how timing of the object-centered to egocentric transformation could affect fast reactions remains to be answered.

\section{Comparison with human fMRI}

Evidence from human imaging studies suggest that in spatial judgment tasks, different brain circuits are involved in egocentric and object-centered encoding of spatial relations (see Introduction). While object-centered spatial judgments activate mainly areas in the ventral stream, egocentric spatial judgments mostly recruit subregions of the dorsal stream. Additionally, lesions in the dorsal stream lead to deficits in egocentric spatial localizations, e.g., body-centered neglect where patients ignore one side of the space. Whereas, lesions in the ventral stream leads to deficits such as visual agnosia where patients lose their ability to recognize objects or spatial relation between different objects. However there is growing evidence from fMRI results which directly compared the two tasks, repeatedly pointed to overlapping structures for egocentric and allocentric encoding in the dorsal stream (Galati et al., 2000; Thaler and Goodale, 2011; Zaehle et al., 2007; Gallivan et al., 2013). Accordingly, some of the subregions of the posterior parietal cortex were active when spatial locations are encoded both in egocentric and allocentric frames of reference. Furthermore, Fink and colleagues (Fink et al., 2003) showed that egocentric and object-centered reference frames interact in the human frontoparietal network. Human subjects were asked to perform object-centered spatial judgment task while they were receiving galvanic vestibular stimulation (manipulation of egocentrci reference frame). A significant interaction of the stimulation and neural mechanism underlying object-centered spatial judgment was observed in right posterior parietal and ventral premotor cortices.

Recent human imaging studies investigated the egocentric versus object-centered reach movement planning and execution. Thaler and Goodale (Thaler and Goodale, 2011a) showed 
that in addition to boosting the BOLD activity in the same right frontoparietal network which was involved in the egocentric movement, the object-centered task recruit subregions of the left intra-parietal sulcus in the posterior parietal cortex of the dorsal stream and lateral occipital cortex of the ventral stream. Chen and colleagues (Chen et al., 2014) studied directional selectivity of the BOLD signal for egocentric versus object-centered encodings of visual cue and movement plan. During visual memory period, they observed significant object-centered encoding in subregions of the temporal and early visual cortices. However, in movement planning phase, the occipital, parietal and frontal areas showed egocentric encoding of the movement goal.

There have not been any imaging studies on monkeys to pinpoint subregions of the parietal and frontal cortices which encode both egocentric and object-centered spatial locations. In the

present work, we showed that individual cells of subregions of the parietal and frontal cortices in the dorsal stream show tuning to object-centered location of the visual cues and reach movement goals. Object-centered encoding of the cue stimulus agrees with the results from human perceptual spatial judgment studies where they reported object-centered and egocentric circuits overlap in the parietal cortex (Committeri et al., 2004; Neely et al., 2008; Galati et al., 2000). The fact that in our data movement plan is encoded in egocentric reference frame both in PRR and PMd, fits nicely with result of the recent human imaging study by Chen and colleagues (Chen et al., 2014).

\section{Latency difference between PRR and PMd in visual cue encoding}

From our SNR analysis in Exp I, we observed that in response to the cue presentation, selectivity to the cue position evolved first in PMd and later in PRR (see Figure 7b). In Exp I, in half of the trials cue and the movement goal have identical spatial locations (reference and decision array congruent trials) whereas in other half of the trials the movement goal was inferred by remapping the cue location (reference and decision objet incongruent trials). Since the congruent and incongruent trials were randomly presented, during reference object and cue presentation epochs of each trial the monkey did not have any knowledge about what type of trial would be presented. Thus, in order to perform the task correctly, initially he had to encode the objectcentered location of the cue which then later in half of the trials had to be remapped to a new egocentric location (incongruent trials). A recent study by Wesstendorf and Gail (Westendorff et 
al., 2010) reported that in anti-reach task when spatial remapping of the cue position was required to infer the movement goal, selectivity for the upcoming movement goal first occurred in PMd and with 27 ms latency in PRR. The latency difference was suggested to be associated with dynamic reorganization of the local neuronal network in each area during sensory to motor transformation. According to this view, the reorganization in PRR was started once the motor goal had been first selected by PMd. Although in that study, cue selectivity and motor goal encoding in egocentric and allocentric references were not distinguished, their line of reasoning could partly explain the earlier selectivity in PMd that we observed in the present data. Since initially every trial should be treated as an incongruent trial, selectivity to the cue position during visual memory period could be a preliminary representation of the inferred motor goal in objectcentered reference frames. In our task design the cue and the target always have the same objectcentered location, in half of the trials the egocentric location of the cue and target are different (incongruent trials). Therefore, neural activity in the first memory period, before presentation of the decision object, can well represent a preliminary motor goal in object-centered reference frame. Later in the delay period after presentation of the decision object, the definite motor goal is inferred by remapping the preliminary motor goal into egocentric reference frame.

On the other hand, given that in the early response of PMd, during cue presentation, most neurons encode in the egocentric reference frame and later at the end of the visual memory period changes to object-centered encoding, it can well be a visual response to presentation of the physical cue stimulus; with the present experiment we cannot distinguish between the alternative possibilities.

\section{Size-invariant spatial encoding}

During visually guided reach planning, when reaches are directed towards objects then object shape and size partly determine the relevant space for motor planning. To our knowledge, it has not yet deeply studied how the neural activity during reach planning, is modulated by the object size. In a recent study on human subjects, Tarantino and colleagues showed that in a reach to grasp task, size of the target object not only affects kinematics of the movement, it also modulates electroencephalogram activity in the frontoparietal areas of human subjects (Tarantino et al., 2014). Other studies showed that single neurons of area V6a of the posterior parietal cortex (Fattori et al., 2012) and PMv of the frontal cortex (Raos et al., 2004) of monkeys were sensitive 
to intrinsic features of target objects (such as shape) during reach to grasp tasks. Here in Exp II, we addressed this question at the single cell level in the reach planning network in monkeys (areas MIP and PMd). A subset of neurons in both areas scaled their spatial selectivity with the size of the object so that they had size-invariant spatial tuning for the cue position relative to the object. Our result is in agreement with the previous finding from human EEG. It further provides neuronal evidence and shows how individual neurons contribute to the modulation of the activity in both areas.

\section{Scalable and dynamic selectivity of individual cells in PRR and PMd}

Areas PRR and PMd are believed to encode spatial relations which are necessary for movement planning as well as different parameters of the pending reach movement. Neurons in these areas are known to encode task related spatial parameters in egocentric fashion. From Exp I, we found that individual cells in PRR and PMd can also be finely tuned to object-centered position of the cue and movement goal, independently of the absolute location of the object. Such spatial selectivity indicates that when the object is displaced, the spatial selectivity of the objectcentered cells shifts towards the new location of the target object and results in a spatial selectivity which is independent of the absolute location of the object. Size-invariant spatial tunings in Exp II, shows that cells not only shift their spatial selectivity, the width of the selectivity profile is also scaled with the size of the target object. This is the first study that provides direct evidence reflecting such flexible spatial encoding, showing that neurons in PRR and PMd adjust their spatial selectivity to the behaviorally relevant spatial space.

In the present behavioral paradigm, at the early stage of each trial, onset of the cue stimulus

provides object-centered position of the target and the behavioral task was designed to make the object-centered reference frame, the most critical spatial reference at this phase of the trial. Later in the trial, movement goal needs to be inferred by integrating decision object location and the object-centered location of the target. Finally in order to execute the movement reach goal must be encoded in an egocentric reference frame and thus at the late phase of the trial the egocentric reference frame is behaviorally the most relevant spatial reference. Accordingly, as Exp I shows (see Figure 7a and b), during and after cue presentation, location of the cue is predominantly encoded in the object-centered reference frame and later during movement planning, both areas change their encoding to egocentric location of the reach goal. The neural selectivity nicely 
reflects the behaviorally relevant reference frame in different phases of the trial. Present data clearly shows that different task related parameters are encoded in different reference frames according to the behavioral relevance of the reference frame. Altogether, current results suggest a flexible, scalable and dynamic spatial selectivity in both areas which is adjusted to the behavioral needs.

\section{References}

1. Andersen RA, Essick GK, Siegel RM (1985) Encoding of spatial location by posterior parietal neurons. Science 230: 456-458.

2. Avillac M, Deneve S, Olivier E, Pouget A, Duhamel JR (2005) Reference frames for representing visual and tactile locations in parietal cortex. Nat Neurosci 8: 941-949.

3. Batista AP, Buneo CA, Snyder LH, Andersen RA (1999) Reach plans in eye-centered coordinates. Science 285: 257-260.

4. Batista AP, Santhanam G, Yu BM, Ryu SI, Afshar A, Shenoy KV (2007) Reference frames for reach planning in macaque dorsal premotor cortex. J Neurophysiol 98: 966-983.

5. Bremner LR, Andersen RA (2012) Coding of the reach vector in parietal area 5d. Neuron 75: 342-351.

6. Bremner LR, Andersen RA (2014) Temporal Analysis of Reference Frames in Parietal Cortex Area 5d during Reach Planning. J Neurosci 34: 5273-5284.

7. Buneo CA, Batista AP, Jarvis MR, Andersen RA (2008) Time-invariant reference frames for parietal reach activity. Exp Brain Res 188: 77-89.

8. Buneo CA, Jarvis MR, Batista AP, Andersen RA (2002) Direct visuomotor transformations for reaching. Nature 416: 632-636.

9. Burgess N (2006) Spatial memory: how egocentric and allocentric combine. Trends Cogn Sci 10: 551-557.

10. Byrne PA, Crawford JD (2010) Cue reliability and a landmark stability heuristic determine relative weighting between egocentric and allocentric visual information in memory-guided reach. J Neurophysiol 103: 3054-3069. 
11. Caminiti R, Johnson PB, Burnod Y, Galli C, Ferraina S (1990) Shift of preferred directions of premotor cortical cells with arm movements performed across the workspace. Exp Brain Res 83: 228-232.

12. Caminiti R, Johnson PB, Galli C, Ferraina S, Burnod Y (1991) Making arm movements within different parts of space: the premotor and motor cortical representation of a coordinate system for reaching to visual targets. J Neurosci 11: 1182-1197.

13. Chafee MV, Averbeck BB, Crowe DA (2007) Representing spatial relationships in posterior parietal cortex: single neurons code object-referenced position. Cereb Cortex 17: 2914-2932.

14. Chang SW, Snyder LH (2010) Idiosyncratic and systematic aspects of spatial representations in the macaque parietal cortex. Proc Natl Acad Sci U S A 107: 7951-7956.

15. Chang SW, Snyder LH (2012) The representations of reach endpoints in posterior parietal cortex depend on which hand does the reaching. J Neurophysiol 107: 2352-2365.

16. Chen Y, Byrne P, Crawford JD (2011) Time course of allocentric decay, egocentric decay, and allocentric-to-egocentric conversion in memory-guided reach. Neuropsychologia 49: 49-60.

17. Chen Y, Monaco S, Byrne P, Yan X, Henriques DY, Crawford JD (2014) Allocentric versus Egocentric Representation of Remembered Reach Targets in Human Cortex. J Neurosci 34: 12515-12526.

18. Cohen YE, Andersen RA (2002) A common reference frame for movement plans in the posterior parietal cortex. Nat Rev Neurosci 3: 553-562.

19. Committeri G, Galati G, Paradis AL, Pizzamiglio L, Berthoz A, LeBihan D (2004) Reference frames for spatial cognition: different brain areas are involved in viewer-, object, and landmark-centered judgments about object location. J Cogn Neurosci 16: 1517-1535.

20. Crowe DA, Averbeck BB, Chafee MV (2008) Neural ensemble decoding reveals a correlate of viewer- to object-centered spatial transformation in monkey parietal cortex. J Neurosci 28: 5218-5228.

21. Cui H, Andersen RA (2011) Different representations of potential and selected motor plans by distinct parietal areas. J Neurosci 31: 18130-18136.

22. Efron B (1979) Bootstrap Methods: Another Look at the Jackknife. The annals of Statistics 7(1):1-26.

23. Fattori P, Breveglieri R, Raos V, Bosco A, Galletti C (2012) Vision for action in the macaque medial posterior parietal cortex. J Neurosci 32: 3221-3234.

24. Fink GR, Marshall JC, Weiss PH, Stephan T, Grefkes C, Shah NJ, Zilles K, Dieterich M (2003) Performing allocentric visuospatial judgments with induced distortion of the 
egocentric reference frame: an fMRI study with clinical implications. Neuroimage 20: 1505-1517.

25. Gail A, Andersen RA (2006) Neural dynamics in monkey parietal reach region reflect context-specific sensorimotor transformations. J Neurosci 26: 9376-9384.

26. Gail A, Klaes C, Westendorff S (2009) Implementation of spatial transformation rules for goal-directed reaching via gain modulation in monkey parietal and premotor cortex. J Neurosci 29: 9490-9499.

27. Galati G, Lobel E, Vallar G, Berthoz A, Pizzamiglio L, Le Bihan D (2000) The neural basis of egocentric and allocentric coding of space in humans: a functional magnetic resonance study. Exp Brain Res 133: 156-164.

28. Hwang EJ, Andersen RA (2012) Spiking and LFP activity in PRR during symbolically instructed reaches. J Neurophysiol 107: 836-849.

29. Johnson PB, Ferraina S, Bianchi L, Caminiti R (1996) Cortical networks for visual reaching: physiological and anatomical organization of frontal and parietal lobe arm regions. Cereb Cortex 6: 102-119.

30. Kuang S, Morel P Gail A. Planning movements in visual and physical space in monkey posterior parietal cortex. 2014.

Ref Type: Generic

31. Maier A, Wilke M, Aura C, Zhu C, Ye FQ, Leopold DA (2008) Divergence of fMRI and neural signals in V1 during perceptual suppression in the awake monkey. Nat Neurosci 11: 1193-1200.

32. Marzocchi N, Breveglieri R, Galletti C, Fattori P (2008) Reaching activity in parietal area V6A of macaque: eye influence on arm activity or retinocentric coding of reaching movements? Eur J Neurosci 27: 775-789.

33. McGuire LM, Sabes PN (2011) Heterogeneous representations in the superior parietal lobule are common across reaches to visual and proprioceptive targets. J Neurosci 31: 6661-6673.

34. Milner AD, Goodale MA (2008) Two visual systems re-viewed. Neuropsychologia 46: 774-785.

35. Moorman DE, Olson CR (2007) Impact of experience on the representation of objectcentered space in the macaque supplementary eye field. J Neurophysiol 97: 2159-2173.

36. Mullette-Gillman OA, Cohen YE, Groh JM (2005) Eye-centered, head-centered, and complex coding of visual and auditory targets in the intraparietal sulcus. J Neurophysiol 94: 2331-2352. 
37. Neely KA, Tessmer A, Binsted G, Heath M (2008) Goal-directed reaching: movement strategies influence the weighting of allocentric and egocentric visual cues. Exp Brain Res 186: 375-384.

38. Olson CR, Gettner SN (1995) Object-centered direction selectivity in the macaque supplementary eye field. Science 269: 985-988.

39. Olson CR, Gettner SN (1999) Macaque SEF neurons encode object-centered directions of eye movements regardless of the visual attributes of instructional cues. J Neurophysiol 81: 2340-2346.

40. Olson CR, Gettner SN, Ventura V, Carta R, Kass RE (2000) Neuronal activity in macaque supplementary eye field during planning of saccades in response to pattern and spatial cues. J Neurophysiol 84: 1369-1384.

41. Olson CR, Tremblay L (2000) Macaque supplementary eye field neurons encode objectcentered locations relative to both continuous and discontinuous objects. J Neurophysiol 83: 2392-2411.

42. Pesaran B, Nelson MJ, Andersen RA (2006) Dorsal premotor neurons encode the relative position of the hand, eye, and goal during reach planning. Neuron 51: 125-134.

43. Pouget A, Deneve S, Duhamel JR (2002) A computational perspective on the neural basis of multisensory spatial representations. Nat Rev Neurosci 3: 741-747.

44. Pouget A, Snyder LH (2000) Computational approaches to sensorimotor transformations. Nat Neurosci 3 Suppl: 1192-1198.

45. Raos V, Umilta MA, Gallese V, Fogassi L (2004) Functional properties of grasping-related neurons in the dorsal premotor area F2 of the macaque monkey. J Neurophysiol 92: 19902002.

46. Sabes PN, Breznen B, Andersen RA (2002) Parietal representation of object-based saccades. J Neurophysiol 88: 1815-1829.

47. Snyder LH, Batista AP, Andersen RA (1997) Coding of intention in the posterior parietal cortex. Nature 386: 167-170.

48. Snyder LH, Grieve KL, Brotchie P, Andersen RA (1998) Separate body- and worldreferenced representations of visual space in parietal cortex. Nature 394: 887-891.

49. Tarantino V, De ST, Straulino E, Begliomini C, Castiello U (2014) Object size modulates fronto-parietal activity during reaching movements. Eur J Neurosci 39: 1528-1537.

50. Thaler L, Goodale MA (2011a) Neural substrates of visual spatial coding and visual feedback control for hand movements in allocentric and target-directed tasks. Front Hum Neurosci 5: 92. 
51. Thaler L, Goodale MA (2011b) Reaction times for allocentric movements are 35 ms slower than reaction times for target-directed movements. Exp Brain Res 211: 313-328.

52. Thaler L, Goodale MA (2011c) The role of online visual feedback for the control of targetdirected and allocentric hand movements. J Neurophysiol 105: 846-859.

53. Vallar G, Lobel E, Galati G, Berthoz A, Pizzamiglio L, Le BD (1999) A fronto-parietal system for computing the egocentric spatial frame of reference in humans. Exp Brain Res 124: 281-286.

54. Westendorff S, Klaes C, Gail A (2010) The cortical timeline for deciding on reach motor goals. J Neurosci 30: 5426-5436.

55. Zaehle T, Jordan K, Wustenberg T, Baudewig J, Dechent P, Mast FW (2007) The neural basis of the egocentric and allocentric spatial frame of reference. Brain Res 1137: 92-103.

56. Zipser D, Andersen RA (1988) The role of the teacher in learning-based models of parietal area 7a. Brain Res Bull 21: 505-512. 


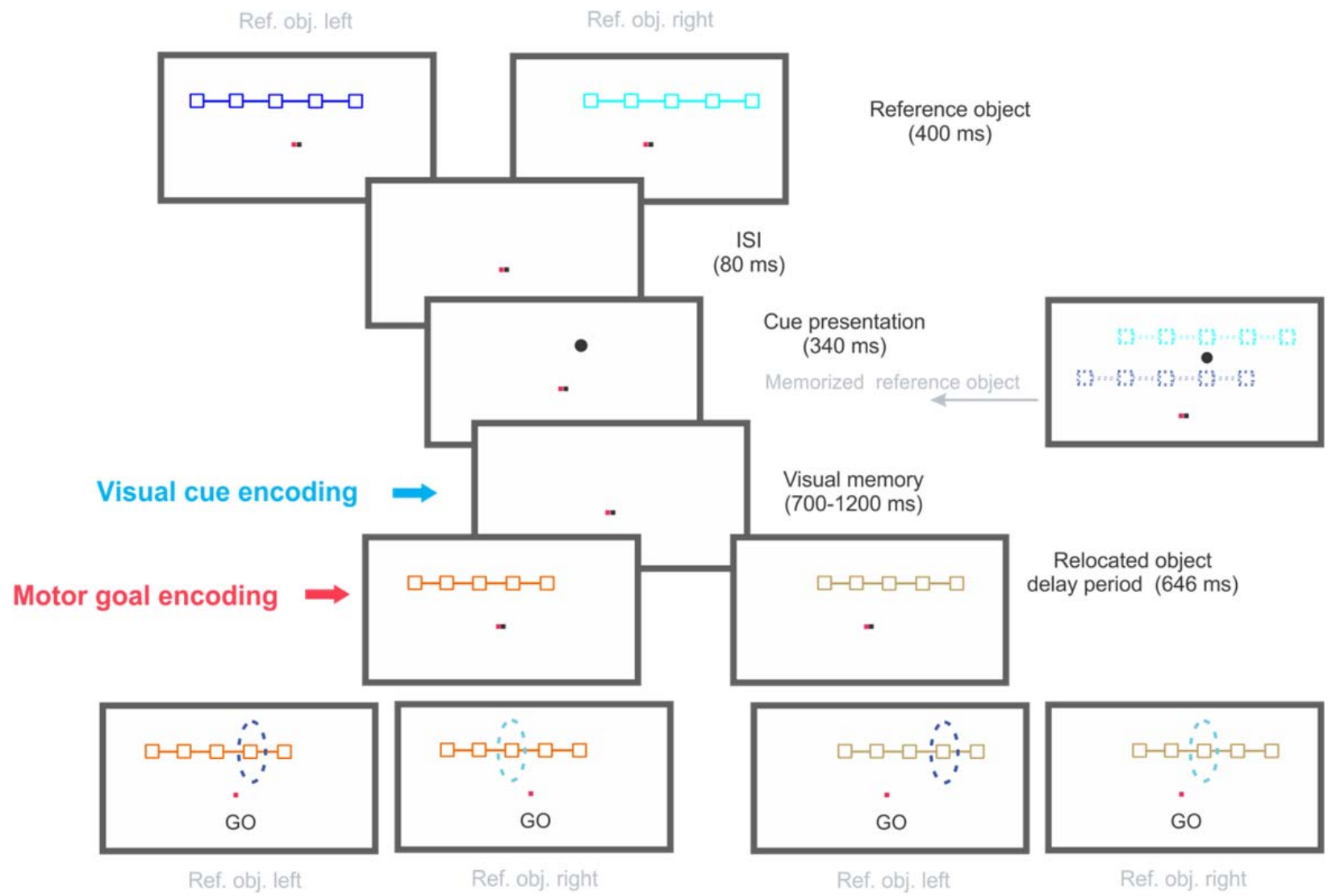

Figure 1 Object-based reach task. After successful eye and hand fixation, a reference object is flashed on the screen. The reference object is an array of five interconnected boxes. The boxes represent potential cue positions. Shortly after offset of the reference object, cue is presented. Presentation of the cue is followed by the visual memory period after which the decision object is presented. Presentation of the decision object is succeeded by a constant delay period during which the monkey has to withhold fixation and do not initiate movement. Offset of the hand fixation stimulus is the GO signal, indicating to the monkey to release hand fixation and reach to the target. 


\section{(a) Exp I}

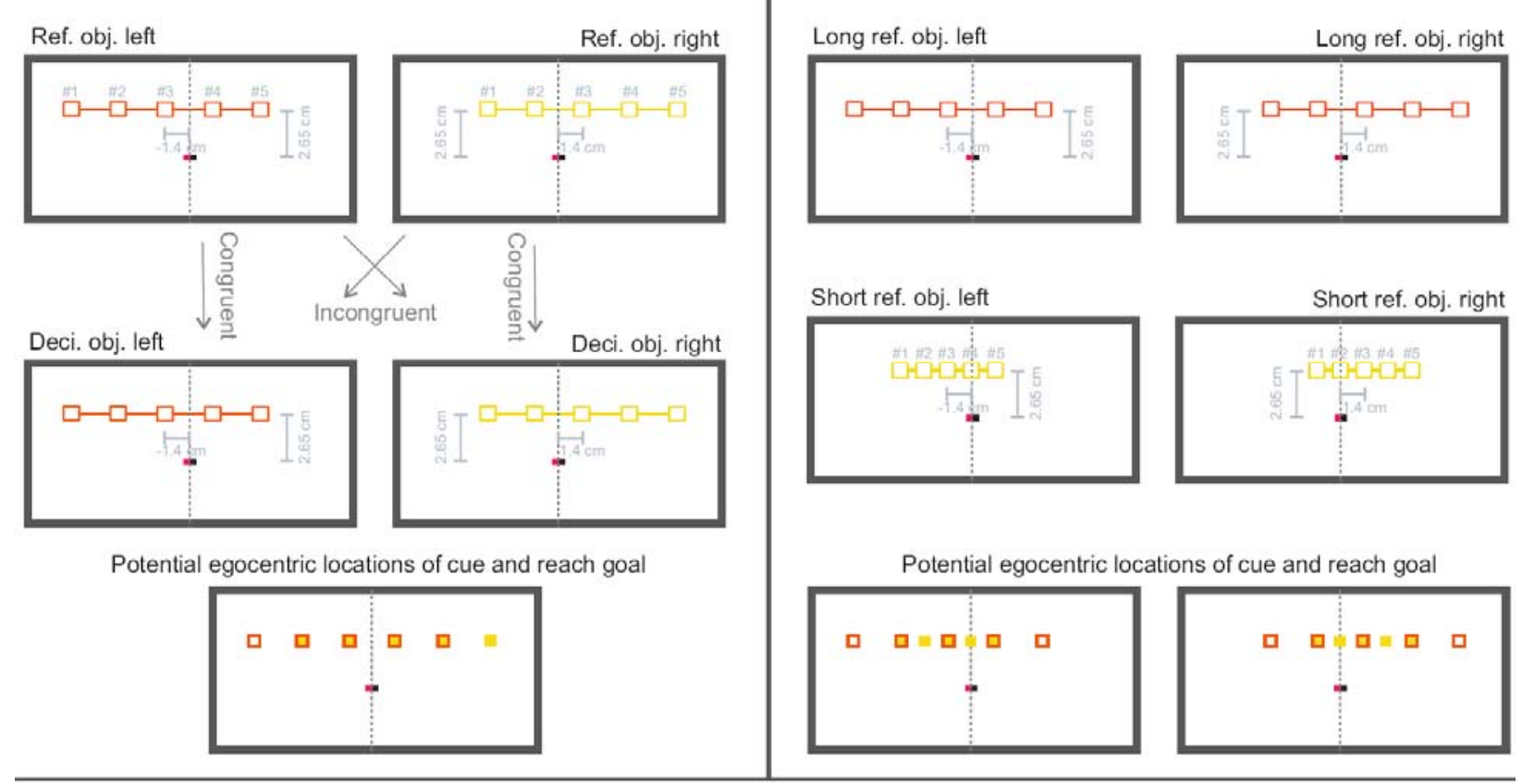

Figure 2 Spatial configuration of the stimuli. (a) In Exp I, reference and decision object are identical in shape and could have two egocentric locations on the left and right side of the screen. When the object is presented at the right side, box \#2 to \#5 overlaps with box \#1 to \#4 when the it is presented at the left side. As illustrated by arrows, each location of the reference object could be randomly succeeded by one of the two locations of the decision object, congruent or incongruent to the location of the reference object. (b) In Exp II, long and short reference object could have two egocentric locations. Reference object boxes overlap as follows: Long left and long right reference objects boxes \#2 to \#5 with \#1 to \#4; Short left and short right reference objects boxes \#3 to \#5 with \#1 to \#3; Long left (right) and short left (right) reference objects boxes \#2, \#3 and \#4 with \#1, \#3 and \#5. Decision object had two egocentric locations (not shown here), both were incongruent to the location of the reference object. 
(a)

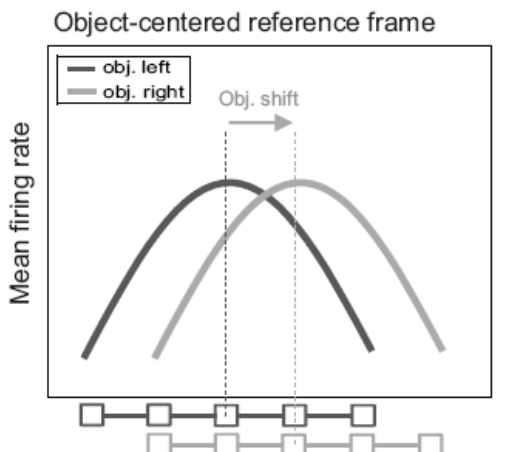

Egocentric reference frame

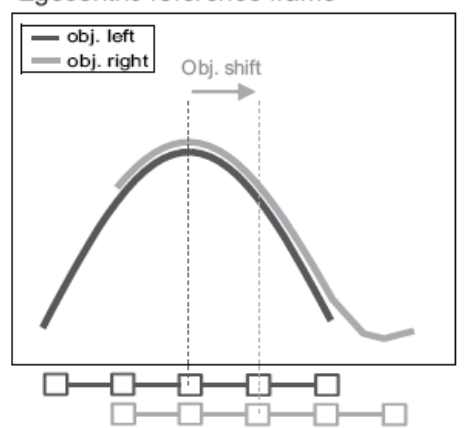

Intermediate reference frame

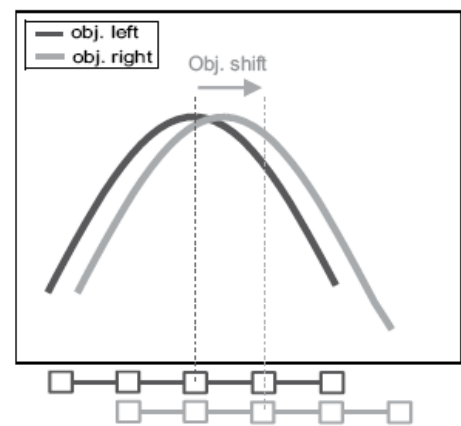

Cue or target locations relative to body in ' $\mathrm{X}$ ' dimension

(b)

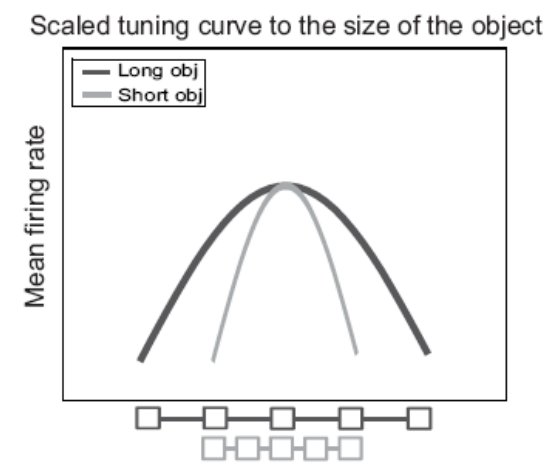

Cue locations relative to body in ' $\mathrm{X}$ ' dimension

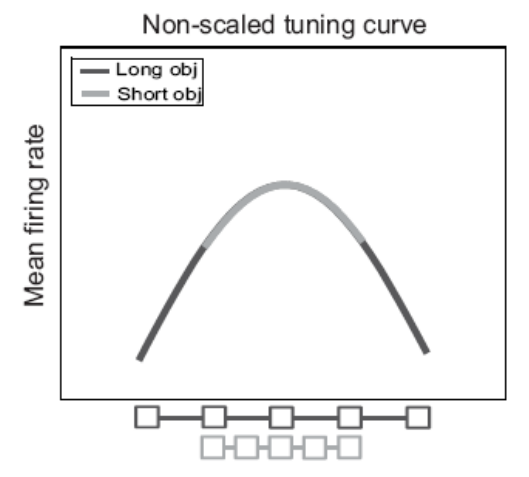

Cue locations relative to body in ' $\mathrm{X}$ ' dimension

Figure 3 Ideal cell response. (a) Tuning curve of an ideal object-centered neuron shifts along with the shift of the object. For an intermediate reference frame, shift of the tuning curve is less than shift of the object on the screen. Tuning curve of an egocentric cell, does not shift on the screen when the object is relocated. (b) When the tuning of the cell is scale to the size of the object, the width of the tuning curve is scaled while the shape of the tuning curve is preserved. When the tuning curve is not scaled to the size of the object, in short object trials the tuning curve is samples with higher spatial resolution. 
(a)

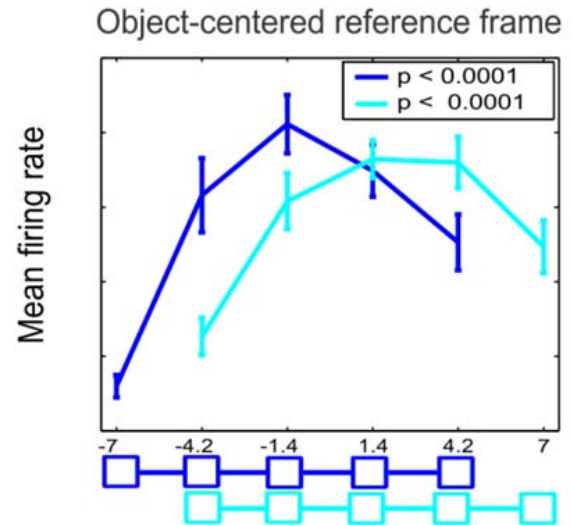

Cue relative to body $(\mathrm{cm})$

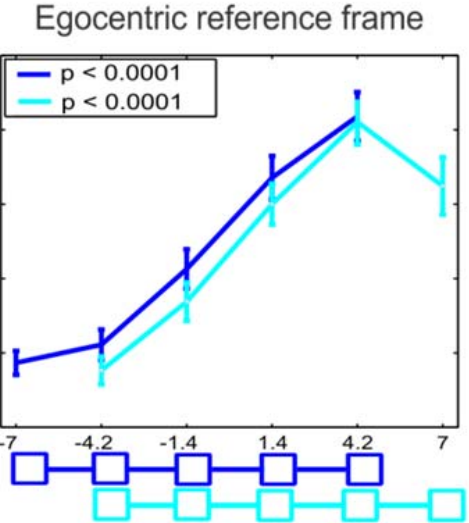

Cue relative to body $(\mathrm{cm})$
Intermediate reference frame

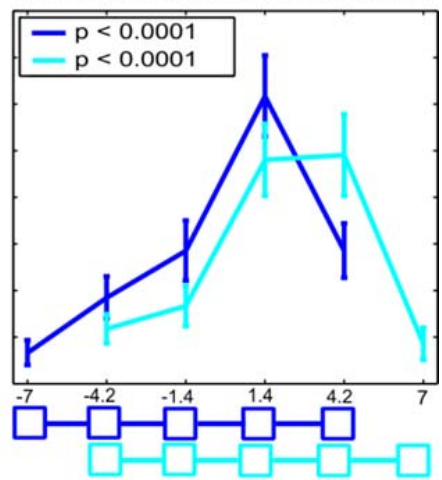

Cue relative to body $(\mathrm{cm})$

(b)
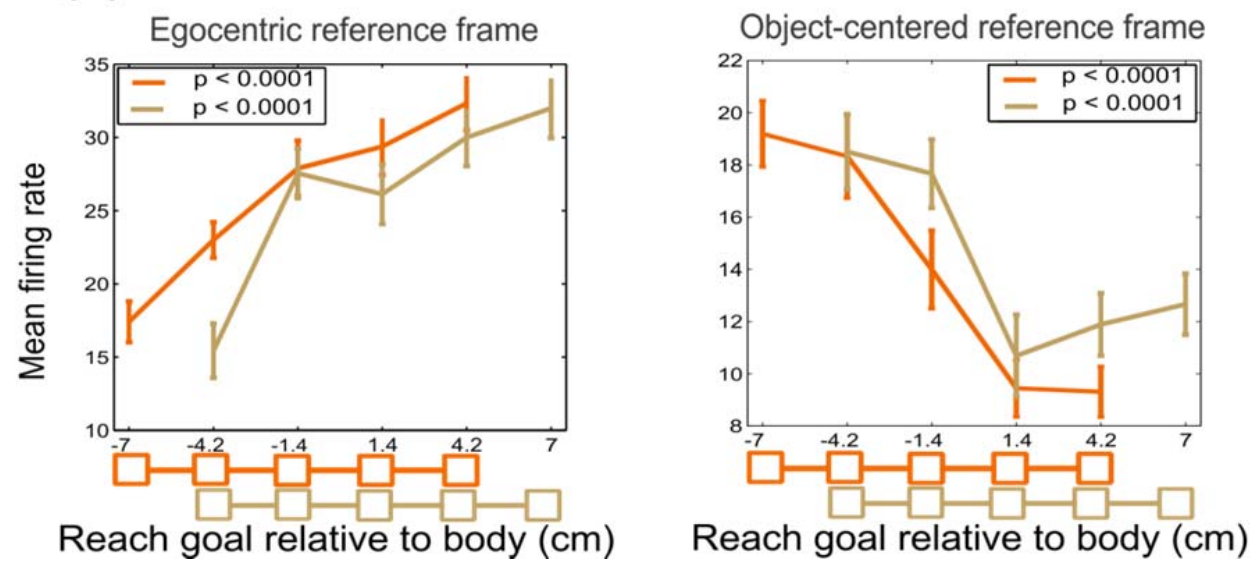

Figure 4 Example cell responses during memory period and movement planning. Neurons in PRR and PMd (a) encode location of the cue and (b) location of the reach goal in object-centered as well as egocentric reference frame. Some neurons use intermediate reference frame (mixed object-centered and egocentric) which is qualified by partial shift of the tuning curve. 
PRR
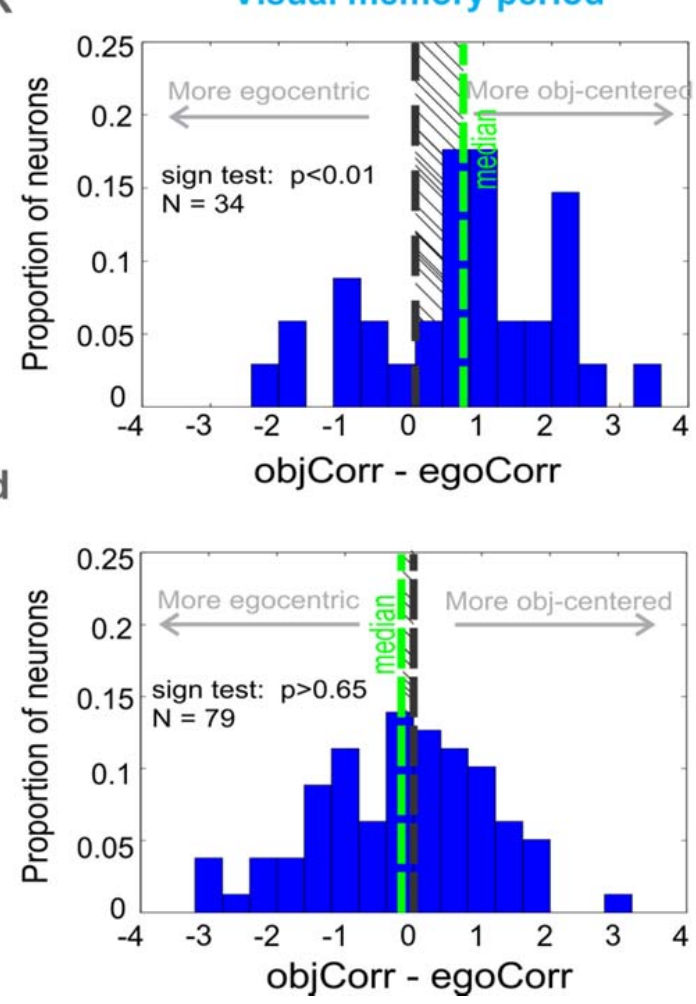

Motor goal planning period
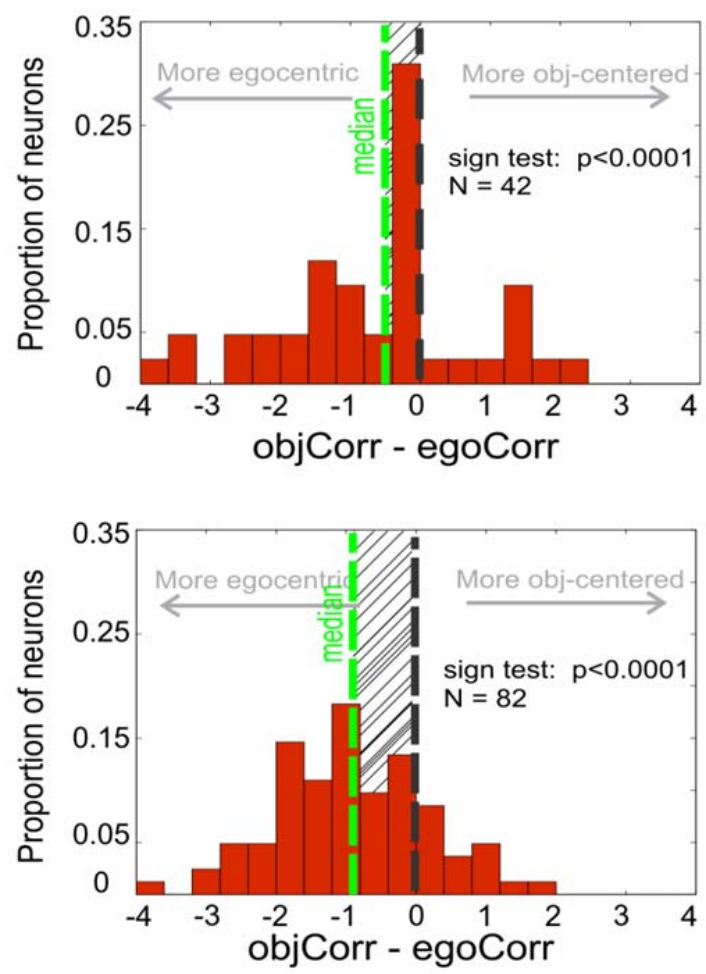

Figure 5 Distribution of the difference of the two correlation coefficients. Based on the similarity of the tuning shape, during visual memory encoding, majority of PRR neurons encode the cue location in object-centered reference frame (qualified by significant shift of the distribution towards positive values) whereas in PMd both object-centered and egocentric encoding were represented equally. During movement planning period majority of neurons in both areas encode the motor goal in egocentric reference frame. 
(a)
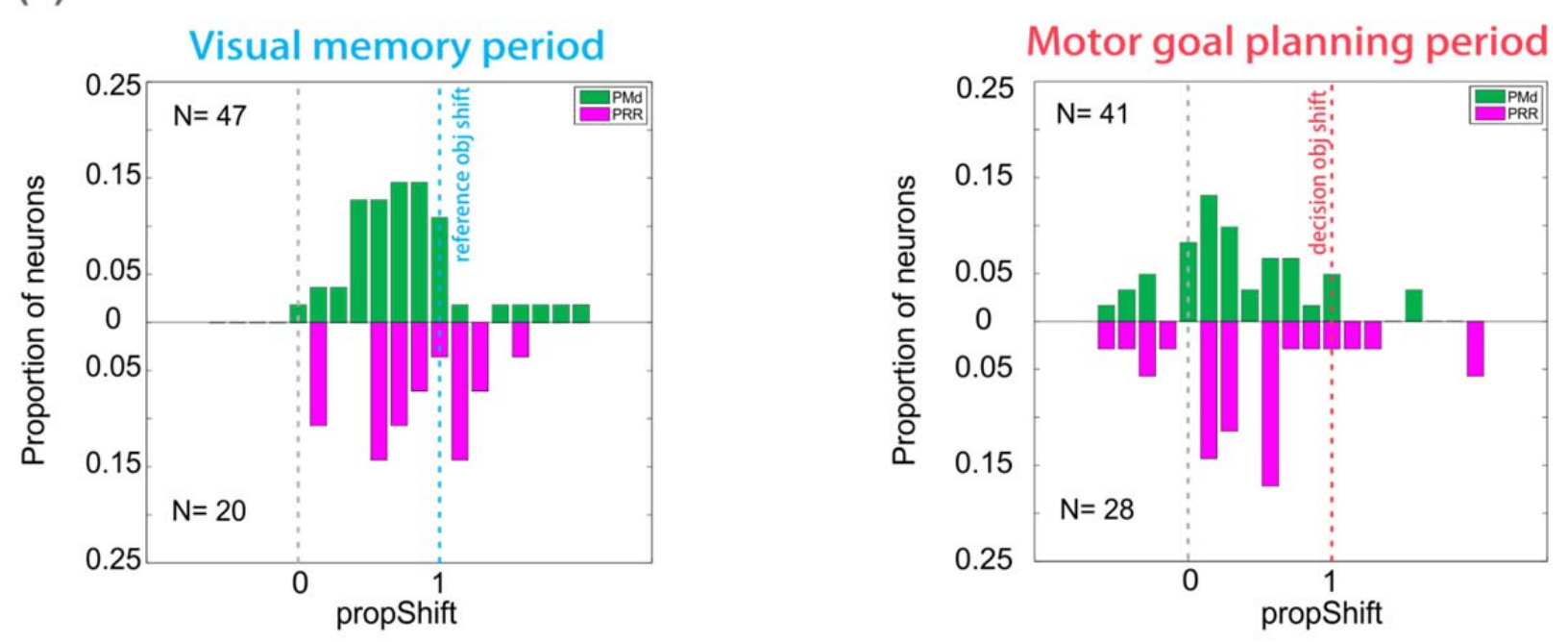

(b)
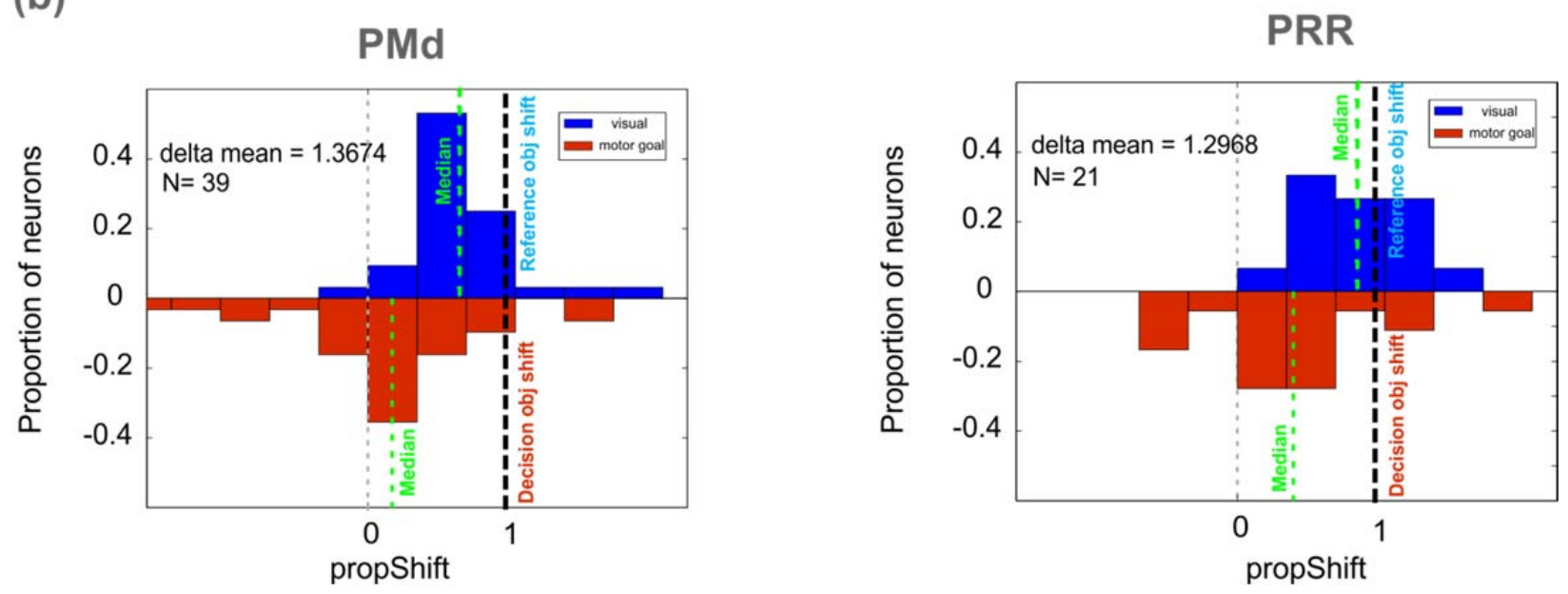

Figure 6 Distribution of the shift of the tuning curves. (a) in both areas, during visual memory period, tuning curve of the neurons shift along with the object shift where as during movement planning the shift is much less compared to the object shift. (b) Paired comparison of the tuning shift during memory period and movement planning confirms that individual cells within each area change their preferred reference frame from object-centered during memory period to egocentric during movement planning. 
(a)

PRR: dynamics of spatial reference frame through the course of a trial

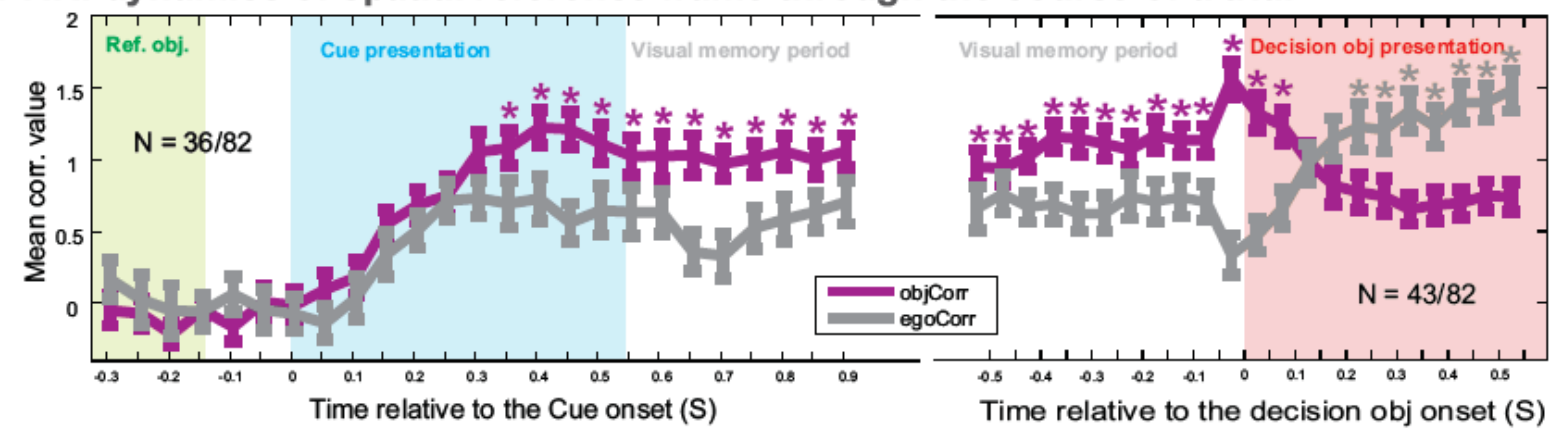

PMd: dynamics of spatial reference frame through the course of a trial

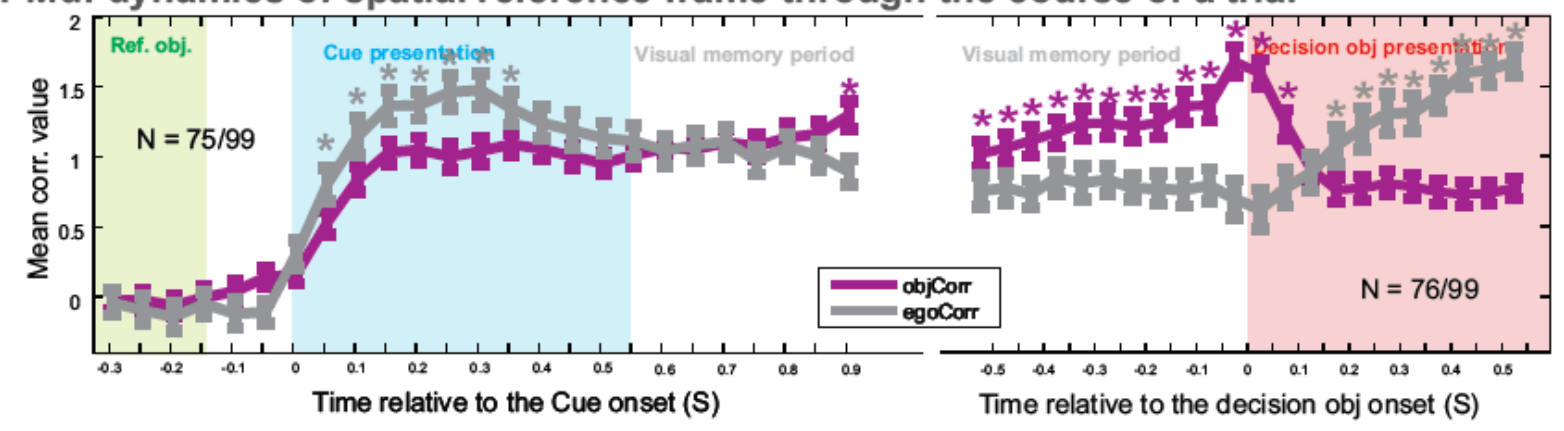

(b)

Strength of neural spatial selectivity through the course of a trial

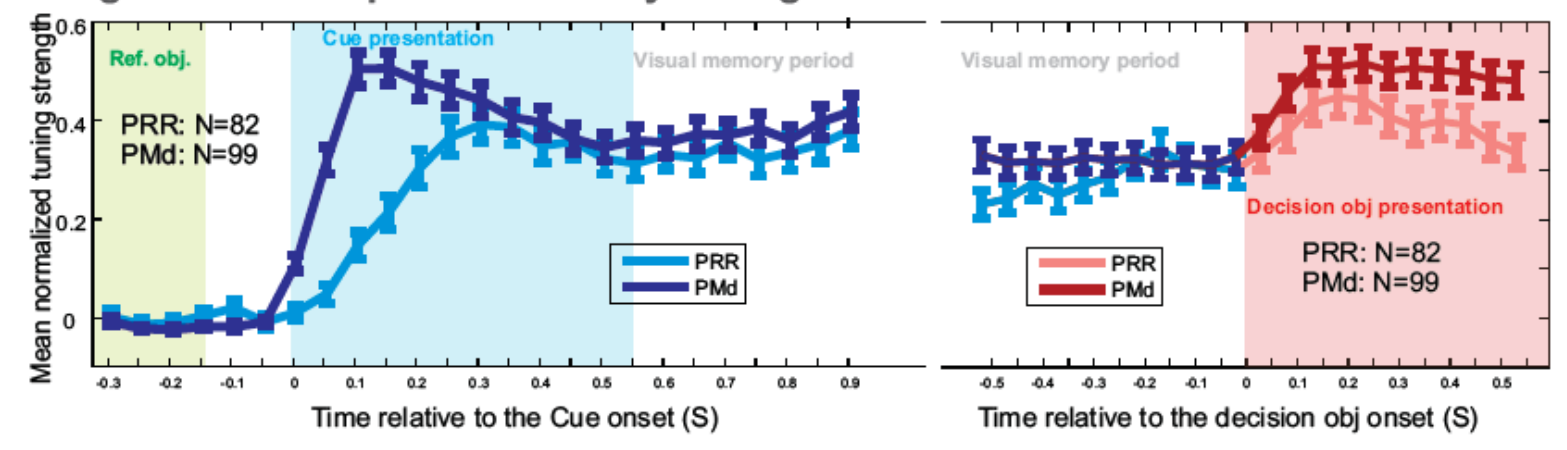

Figure 7 Evolution of reference frames and spatial selectivity through the course of a trial (a) After the cue onset, in PMd the preferred reference frame is initially egocentric during the cue presentation. It gradually changes to the object-centered through the memory period. Whereas in PRR the preferred reference frame is object-centered and this preference does not change through the memory period. At the late memory period, before the onset of decision object, the preferred reference frame in both areas is object-centered. After the onset of decision object, the preferred reference frame in both areas gradually changes to egocentric. (b) With onset of the cue 
stimulus, neural selectivity for the cue location occurs first in PMd and later in PRR, with 150200 ms delay. During movement planning selectivity for the location of the motor goal evolves equally in both areas. 
(a)
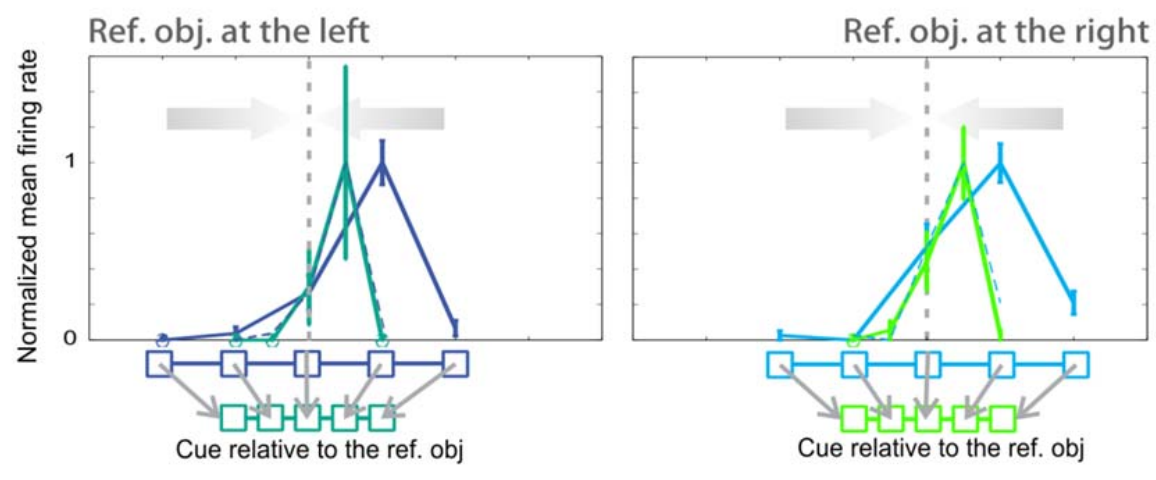

(b)
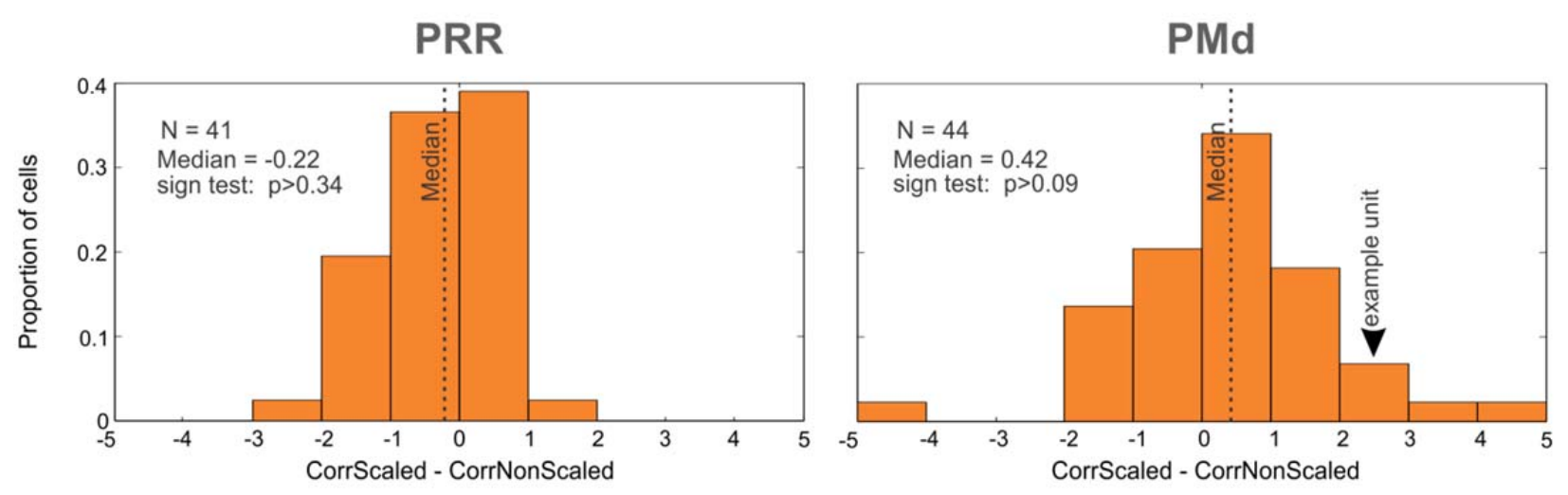

Figure 8 Scaling of tuning curve to the size of the object (a) Example of scaled tuning curve. This figure shows tuning curve of an individual cell to the location of the cue for different reference object size and location. The neurons keeps it pattern of selectivity to different locations on the object, independently of the object location and size. In short object condition, the width of the tuning curve scales to the size of the object so that it perfectly fits to the hypothetically scaled long-object tuning curve (dashed line). (b) Distribution of the difference between CorrScaled and CorrNonScaled. Neurons in both areas, cover a range from fully scaled to non-scaled tuning curves. 


\subsection{Asymmetric generalization in adaptation to target displacement errors in humans and in a neural network model}

When sensorimotor transformation yield to poor motor performance due to external changes in the environment, the brain learns a new mapping between sensory input and motor outputs to bring the performance back to its natural level (see Introduction). In this study, we investigated sensorimotor transformation in the context of motor adaptation. We asked how egocentric reference frames adjust when sensorimotor transformation changes to compensate for a perturbation of the visually instructed reach goal.

We designed a novel behavioral paradigm in which we applied a local perturbation that results in reach target error when the reach target was in a specific part of the space. This experiment was conducted on healthy human subjects. Subjects learned to compensate for the error in the perturbed part of the space. We then probed the reach performance when the reach target was at the unperturbed part of the space. We found that adaptation to the new environmental condition was local. Adaptation effects decayed as a function of the distance between the reach goal and the adapted target. This means that the new sensorimotor transformation, did not induce any offsetting in the center of the frames of reference. 


\title{
Asymmetric generalization in adaptation to target displacement errors in
}

\section{humans and in a neural network model}

\author{
Stephanie Westendorff, ${ }^{1,2}$ Shenbing Kuang, ${ }^{1}$ Bahareh Taghizadeh, ${ }^{1}$ Opher Donchin, ${ }^{3,4}$ \\ and Alexander Gail ${ }^{1,2}$ \\ ${ }^{1}$ German Primate Center, Göttingen, Germany; ${ }^{2}$ Bernstein Center for Computational Neuroscience, Göttingen, Germany; \\ ${ }^{3}$ Department of Biomedical Engineering and Zlotowski Center for Neuroscience, Ben-Gurion University of the Negev, Beer \\ Sheva, Israel; and ${ }^{4}$ Department of Neuroscience, Erasmus Medical College, Rotterdam, The Netherlands
}

Submitted 2 July 2014; accepted in final form 21 January 2015

\begin{abstract}
Westendorff S, Kuang S, Taghizadeh B, Donchin O, Gail A. Asymmetric generalization in adaptation to target displacement errors in humans and in a neural network model. J Neurophysiol 113: 2360-2375, 2015. First published January 21, 2015; doi:10.1152/jn.00483.2014.-Different error signals can induce sensorimotor adaptation during visually guided reaching, possibly evoking different neural adaptation mechanisms. Here we investigate reach adaptation induced by visual target errors without perturbing the actual or sensed hand position. We analyzed the spatial generalization of adaptation to target error to compare it with other known generalization patterns and simulated our results with a neural network model trained to minimize target error independent of prediction errors. Subjects reached to different peripheral visual targets and had to adapt to a sudden fixed-amplitude displacement ("jump") consistently occurring for only one of the reach targets. Subjects simultaneously had to perform contralateral unperturbed saccades, which rendered the reach target jump unnoticeable. As a result, subjects adapted by gradually decreasing reach errors and showed negative aftereffects for the perturbed reach target. Reach errors generalized to unperturbed targets according to a translational rather than rotational generalization pattern, but locally, not globally. More importantly, reach errors generalized asymmetrically with a skewed generalization function in the direction of the target jump. Our neural network model reproduced the skewed generalization after adaptation to target jump without having been explicitly trained to produce a specific generalization pattern. Our combined psychophysical and simulation results suggest that target jump adaptation in reaching can be explained by gradual updating of spatial motor goal representations in sensorimotor association networks, independent of learning induced by a prediction-error about the hand position. The simulations make testable predictions about the underlying changes in the tuning of sensorimotor neurons during target jump adaptation.
\end{abstract}

reach adaptation; target jump; generalization pattern; asymmetry; neural network model

OUR SENSORIMOTOR SYSTEM ADAPTS to environmental changes to sustain proper performance. In goal-directed reaching, adaptation may serve the purposes of reducing the offset between reach endpoint and target position ("target error"; Cameron et al. 2011; Diedrichsen et al. 2005; Magescas et al. 2009), or of readjusting sensory predictions which no longer match the actual sensory feedback about the hand ("prediction error"; Kawato 1999; Mazzoni and Krakauer 2006; Shadmehr and Krakauer 2008). Here we investigate human performance in a target jump task in which error feedback is determined by target error. To constrain possible underlying neural mecha-

Address for reprint requests and other correspondence: A. Gail, German Primate Center, Kellnerweg 4, 37077 Göttingen, Germany (e-mail: agail @ gwdg.de). nisms, we investigate the spatial generalization of adaptation in this task and simulate the human behavior with a recurrent artificial neural network model to infer possible underlying neural changes.

Depending on the perturbation paradigm, target and prediction error will drive adaptation jointly. In the widely used visuomotor rotation or translation tasks (Bedford 1989; Bock 1992; Ghahramani et al. 1996; Imamizu et al. 1995; Krakauer et al. 2000, 2004; Paz et al. 2003; Pine et al. 1996; Vetter et al. 1999; Wise et al. 1998), adaptation is elicited by a perturbed visual feedback (cursor) which induces a discrepancy between seen and actual hand position. This perturbation imposes prediction errors of hand position in the sense that a familiar motor command leads to unexpected sensory consequences (Shadmehr et al. 2010). If the perturbation cannot be compensated during the course of the movement then target errors also occur. Thus, in these tasks, prediction and target errors could both drive adaptation. However, when the target error is experimentally compensated, then adaptation occurs even with prediction error alone (Mazzoni and Krakauer 2006; Tseng et al. 2007). Conversely, target error induced by unnoticed target displacements without prediction error about hand position can also lead to reach adaptation (Cameron et al. 2011; Laurent et al. 2011, 2012; Magescas et al. 2009; Magescas and Prablanc 2006). Thus both target and prediction error can drive adaptation independently, with potentially different underlying neural bases. While adaptation has been investigated intensively for perturbation tasks, which include prediction errors, much less is known about spatial generalization of adaptation driven by target errors in target jump tasks (Magescas et al. 2009).

Sensorimotor adaptation mostly generalizes to nonadapted targets or movements. The generalization pattern of adaptation can help to identify different forms of adaptation and reveal constraints for possible underlying neural mechanisms (Donchin et al. 2003; Imamizu et al. 1995; Krakauer et al. 2000; Magescas et al. 2009; Mattar and Ostry 2010; 2007; Pine et al. 1996; Tanaka et al. 2009; Taylor and Ivry 2013; Thoroughman and Taylor 2005). In our study on target error adaptation, we will focus on three aspects of generalization, introduced in the following paragraphs: range (local or global), frame of reference (translational or rotational), and symmetry (symmetric or skewed). Note, we do not expect these three parameters to necessarily be independent properties of adaptation, but we will present them separately for the purpose of introducing the concepts and analyzing them. 
The spatial range of generalization is expected to relate to the spatial selectivity of underlying neuronal units. The logic is that the properties of those neuronal units which control the movement (Thoroughman and Shadmehr 2000) or which define a sensorimotor map, i.e., the weights between the desired direction of movement and the tuned movement units (Pouget and Snyder 2000), will determine the generalization pattern if they change during adaptation. For example, previous studies have shown that the spatial range of generalization can fundamentally differ when the perturbation to hand feedback is applied either in a rotational or in a translational geometry in visuomotor tasks. Generalization to rotational perturbations was local, i.e., adaptation effects quickly decreased with increasing distance from the trained target (Krakauer et al. 2000; Pine et al. 1996), suggesting narrow tuning functions of the responsible neurons (Tanaka et al. 2009). Generalization to a translational perturbation in contrast was more "global", i.e., adaptation effects occurred for target positions over a wide range (Ghahramani et al. 1996; Taylor and Ivry 2013). Global generalization suggests very broad tuning functions for neurons involved in the relevant sensorimotor transformation or an effect on the neural representation of the origin of the spatial reference frame for reaching (Shadmehr and Wise 2005). A local cross-axial target jump, as we will use it here, can be geometrically interpreted as a rotational as well as a translational perturbation; hence it is open which range of generalization should be expected in such task.

Second, the spatial frame of reference of generalization allows inferring which parameters of the reach kinematics are adapted. The logic again is that adaptation modifies those parts of the neural system, which encode reach parameter in a corresponding frame of reference. For example, previous visuomotor rotation paradigms showed a rotational generalization pattern (Bock 1992; Krakauer et al. 2000), meaning that reaches to unperturbed targets were affected only in direction (polar angle) but not amplitude (radius). Accordingly, one would expect that the neural units affected by adaptation encode direction independent of amplitude. In contrast, when reaches were adapted in a translational fashion in their Cartesian endpoint positions (Ghahramani et al. 1996), this would indicate that units affected by adaptation encode both kinematic reach parameters (direction and amplitude) jointly. Again, since a cross-axial target jump as used in our experiment can be interpreted as a rotational as well as a translational perturbation, it is open which frame of reference for generalization should be expected. We investigate adaptation to target error independent of hand prediction error, and target error is defined via reach endpoints. Hence, we test the hypothesis that target errors in a target jump task induce adaptation of reach endpoints, and therefore might result in translational generalization.

Finally, additional features of generalization with potential implications on the mechanisms of adaptation recently moved into the focus of research. For example, Gonzalez Castro and colleagues (2011) observed generalization patterns in forcefield adaptation that were centered at the direction of the experienced movement rather than the direction of the planned movement, but were otherwise symmetric. Another force-field study, in contrast, showed asymmetric generalization in healthy subjects, but not in cerebellar patients (Izawa et al. 2012). Based on accompanying modeling results, the latter observation let the authors conclude that asymmetric generalization could be a signature of a system that learns by minimizing prediction errors via forward model adaptation. If this is the case, we would have to expect symmetric generalization in tasks which do not induce a prediction error of the hand position, like the target-error task used here.

The first aim of our study was to characterize generalization for a cross-axial target jump with minimal possibility for sensory predictions errors. We designed our task so that prediction error of egocentric arm position would not plausibly be induced. First, our task used the bare arm as the effector and provided continuous unobstructed view of the arm during natural movements. Thus subjects had no need to develop a task-specific internal model of any cursor movement or manipulandum dynamics, but instead could rely on their lifelong trained internal model for estimating arm position. Second, we did not perturb the hand, neither physically nor visually. Thus the subjects had a full, unobstructed view of their free arm movements completely under their own control. The error was exclusively introduced by a jump of the visual target stimulus during the course of the arm movement, but rendered invisible by saccadic suppression.

As a second aim, we used a neural network to simulate the observed adaptation pattern. The main idea of the modeling is to heuristically infer potential underlying neural changes of adaptation, which then can be subject to future neurophysiological testing. Another idea of the modeling is to test the above-mentioned hypothesis that the symmetry of generalization might be determined by the type of error by which the sensorimotor system adapts. Previous network models simulated visuomotor adaptation, which resulted either in asymmetric (skewed) generalization (Izawa et al. 2012) or symmetric generalization (Tanaka et al. 2009; Taylor and Ivry 2013) but used different modeling approaches so that the critical determinant for asymmetric generalization is not clear. We used a recurrent artificial neural network, previously built to simulate the learning of context-specific visuomotor associations (Brozovic et al. 2007) and tested within the same network architecture which connectivity and learning constraints in the model would produce skewed or symmetric generalization.

\section{METHODS}

\section{Subjects}

In total, 48 subjects ( 20 to 58 yr old, 36 women) participated in the experiment, of which 29 ( 20 to 58 yr old, 20 women) were used for the main analyses in this study, 15 were used for a control analysis, and 4 were excluded (see below for details). Detailed written instructions were given to the subjects before the experiment. In a training session, a few minutes before the recording session, subjects could practice the control task without target jump and ask any question not resolved by the written instructions. All subjects were right handed with normal or corrected-to-normal vision and were naive with respect to the objective of the study. Subjects were not informed about the target jump that would be introduced. Experiments adhered to the principles of the Declaration of Helsinki and institutional guidelines. The experiments were reviewed and approved by an institutional review committee of the Georg Elias Müller Institute for Psychology of the University of Göttingen. All subjects gave their written, informed consent. 


\section{Experimental Setup}

All stimuli were presented on an LCD screen (19 in. ViewSonic VX922) facing the subject (i.e., in the fronto-parallel plane) at a distance of $40 \mathrm{~cm}$. Custom-written visual display software $(\mathrm{C}++)$ was controlled via a real-time LabView control program running on a PXI real-time computer (National Instruments). Figure $1 A$ gives an overview about all displayed stimuli. Fixation stimuli (FIX) were displayed in the center of the screen. The fixation stimulus for the gaze consisted of a red square $(0.5 \mathrm{~cm}$ edge length). The hand fixation stimulus (displayed adjacent to the gaze fixation stimulus) consisted of a white square $(0.5 \mathrm{~cm}$ edge length). Reach targets were displayed as gray circular patches $(0.5 \mathrm{~cm}$ radius $)$, saccade targets were displayed as red circular patches $(0.5 \mathrm{~cm}$ radius $)$. Unless otherwise indicated, saccade and reach targets were positioned on a circumference with radius $12 \mathrm{~cm}\left[=17.2^{\circ}\right.$ visual angle (VA) $]$ from the initial starting point for hand placement and saccade fixation. The saccade targets (S1 or S2, Fig. $1 A$ ) were at either $195^{\circ}\left[210^{\circ}\right.$ clockwise $\left.(\mathrm{CW})\right]$ or $285^{\circ}\left(270^{\circ} \mathrm{CW}\right.$; with $0^{\circ}$ to the right). The primary reach target (T1, Fig. $1 A$ ) was always at $60^{\circ}$. The secondary reach target was either $75^{\circ}$ [T2 counterclockwise (ccw)] or $45^{\circ}$ (T2 cw). Probe targets could be displayed at seven locations between $15^{\circ}$ and $105^{\circ}$ (Fig. $1 A,-45^{\circ}$ to $45^{\circ}$ relative to $\left.\mathrm{T} 1\right)$. In interspersed control trials which were not relevant for the present study, the probe target direction remained at $60^{\circ}$, and the extent was one of five chosen in the range $7 \mathrm{~cm}$ to 20.5 $\mathrm{cm}$ from the initial starting position $(5 \mathrm{~cm}$ less to $8.5 \mathrm{~cm}$ more relative to T1, not shown).

Reaching movements were performed without the use of any mechanical devices, so that the hand was unencumbered and unperturbed. Subjects could see their own hand throughout the experiment, so they had direct visual feedback of the hand position during the

\section{E}

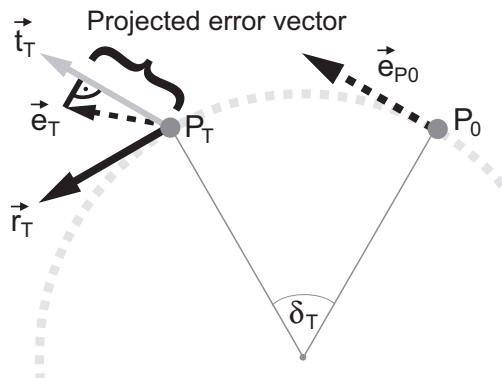

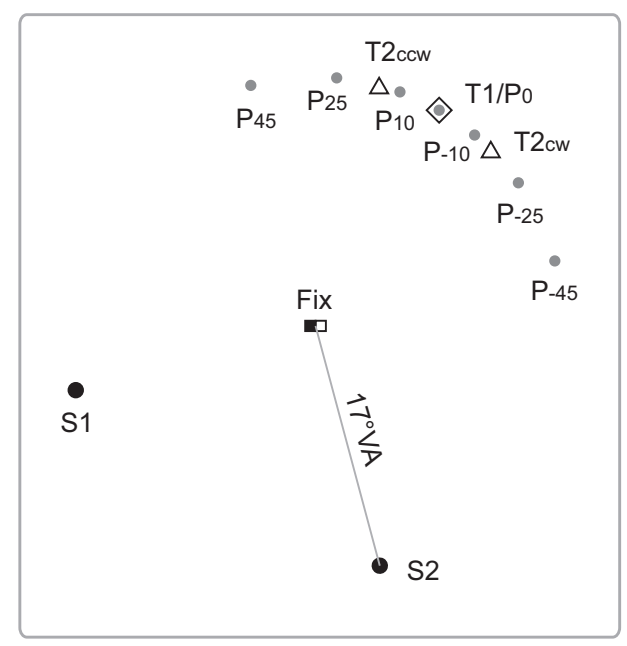

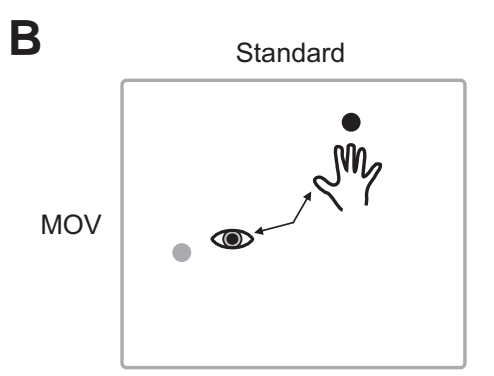

C

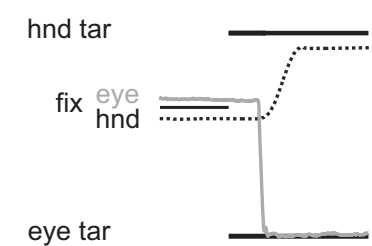

D

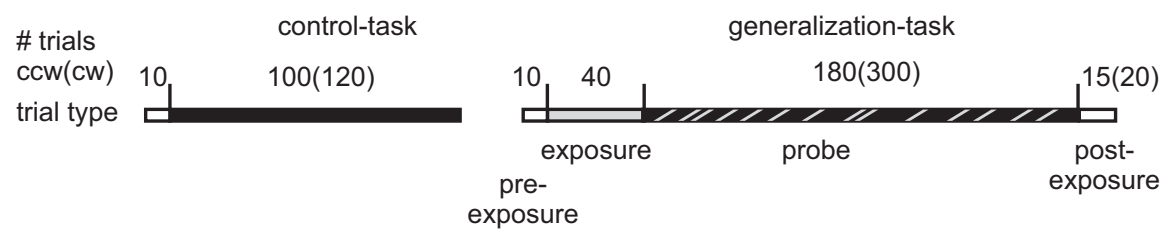


reach without any optical perturbations. Both measures ensured the maximal possible sense of agency. Reach endpoints were registered on a touch screen (IntelliTouch, ELO Systems) mounted immediately in front of the visual display. Gaze positions were registered with an infrared eye-tracking device at $500 \mathrm{~Hz}$ (SMI Sensomotoric Instruments). Eye movements and hand positions were monitored in real time. Subjects used a chin-rest to minimize head movements. In 10 subjects of the CW experiment (see below), we additionally measured hand trajectories at $200 \mathrm{~Hz}$ with a three-dimensional optical motiontracking device (4300 Visualeyez, PTI Systems). One marker was attached to the nail of the index finger of the preferred hand, which was used by all subjects to touch the screen. Motion tracking data were smoothed with a sixth-order Butterworth filter $(10 \mathrm{~Hz}$ cut-off frequency).

\section{Task Design}

In contrast to most previous target-jump paradigms, we investigated reach adaptation and resulting generalization patterns to crossaxis target jumps. This means, we introduced sudden target displacements orthogonal to the reach direction. This allowed a better comparison to the generalization patterns in visuomotor rotation tasks, which are also characterized by cross-axis perturbations. Subjects simultaneously had to reach and saccade to two separate targets (see below for timing information). Saccadic suppression allowed the reach target to jump without subjects noticing. Saccade targets were always on the opposite side of the screen from reach targets. This spatial separation was imposed to prevent interference between saccadic and reach adaptation (Bekkering et al. 1995; De Graaf et al. 1995). When interviewed after completion of the experiment, most subjects (32/46) reported that they had not noticed any perturbation of the target (see below for methodological details and subject selection).

The task consisted of three different trial types: standard (for establishing a baseline), jump (for inducing adaptation), and probe (for measuring the effects of adaptation) trials (Fig. 1, $B$ and $C$ ). In all trial types, after the saccade target appeared, it stayed visible and stationary throughout the trial. In standard trials, the reach target was also visible and stationary until the end of the trial. In jump trials, the reach target remained visible but jumped to a new position during saccade execution. The jump was either in the CCW ( $n=29$ subjects) or the CW $(n=19)$ direction; four subjects conducted the experiments in both directions. The number of trials done by $\mathrm{CCW}$ and $\mathrm{CW}$ subjects was slightly different, but this did not affect our results. Where methodological details differ between the CCW and CW conditions, the CW condition is given in parentheses. In probe trials, the reach target disappeared during the saccade so that reaches were actually executed to the remembered target position.

\section{Task Conditions}

For each subject, the experiment consisted of a sequence of two tasks with a total of six task conditions (Fig. 1D). The control task was used to measure baseline performance. Subjects first conducted 10 standard trials. These were followed by $100(120 \mathrm{CW})$ probe trials. The order of appearance of the probe target positions was randomized and different for each subject. On average, each probe target appeared 10 times.

The generalization task started with 10 standard trials (preexposure period). These were followed by 40 jump trials (exposure period). The jump trials were followed by a generalization period where $\sim 108$ $(\sim 120 \mathrm{CW})$ probe trials were mixed in with $\sim 72(\sim 180 \mathrm{CW})$ jump trials so that the probe trials represented $60 \%(40 \% \mathrm{CW})$ of the generalization period. The jump trials were included in the generalization period as refreshers to ensure that adaptation is maintained. Finally, the subject finished the experiment with a postexposure period consisting of $15(20 \mathrm{CW})$ standard trials.

\section{Behavioral Control and Recording}

Subjects initiated a trial by fixating and touching the fixation stimuli in the center of the screen (FIX; Fig. 1A). After a variable delay $(0.5$ to $1.0 \mathrm{~s})$, the fixation stimuli disappeared ("go-signal"), while simultaneously the reach target and the saccade target appeared. Subjects had $700 \mathrm{~ms}$ to react to the appearance of the eye and hand targets by initiating a saccade. Saccade initiation was defined as the time when the gaze signal exited the eye-tolerance window. The gaze had to leave the tolerance window in a direction within $\pm 45^{\circ}$ of the direction of the saccade target, otherwise the trial was aborted. The target jump was triggered 24-40 ms after saccade initiation (depending on when the saccade occurred relative to the refresh cycle of the screen). Within $500 \mathrm{~ms}$ after saccade initiation, subjects had to position gaze and hand within the tolerance window of the respective targets. After gaze and hand entered the tolerance window at the target position, both had to stay within the tolerance windows for additional $500 \mathrm{~ms}$ to successfully finish the trial. Otherwise the trial was aborted. The subject had to touch the screen within a radius of $2.5 \mathrm{~cm}\left(3.6^{\circ} \mathrm{VA}\right)$ of the hand fixation stimulus and within a radius of $5 \mathrm{~cm}\left(7^{\circ} \mathrm{VA}\right)$ of the reach target and maintain contact with the screen within that radius. The reach target tolerance was chosen to be relatively large such that it would not impose constraints onto adaptation or generalization

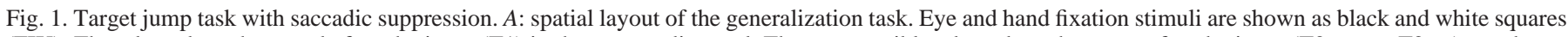

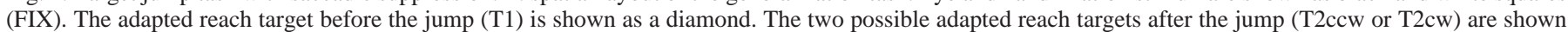

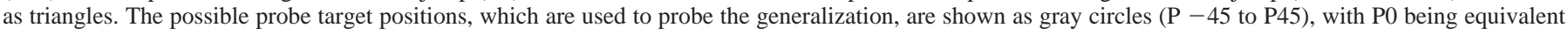

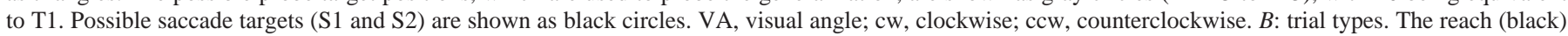

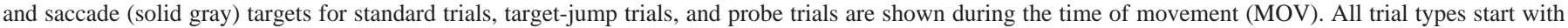

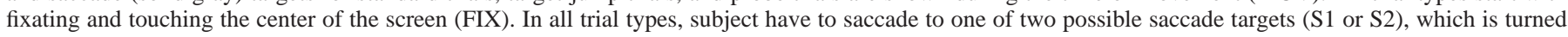

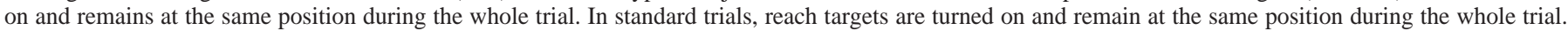

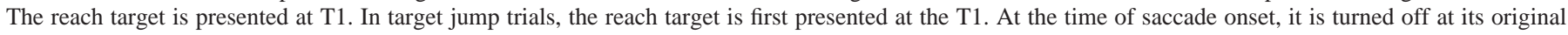

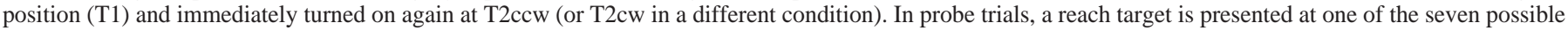

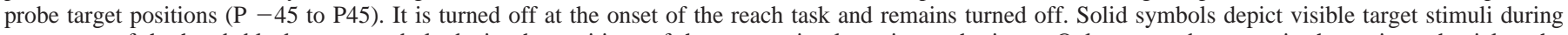

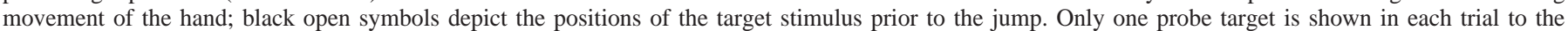

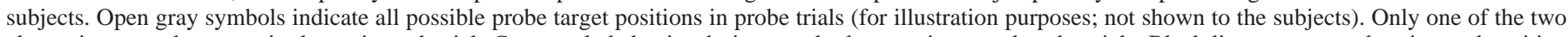

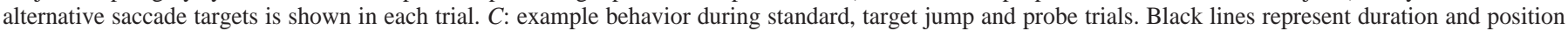

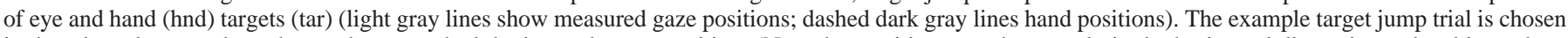

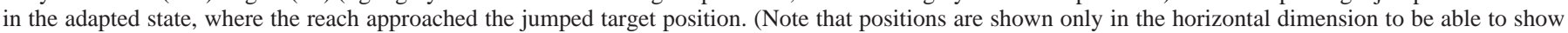

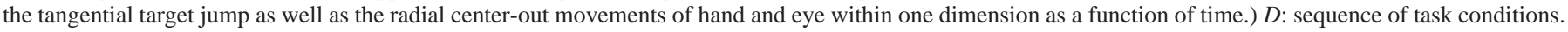

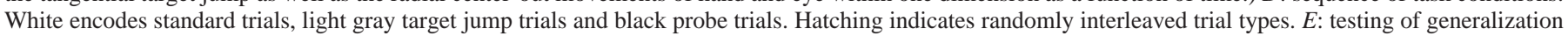

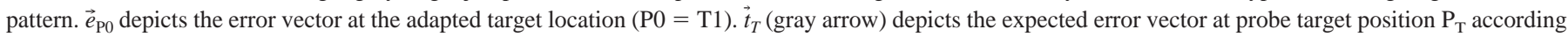

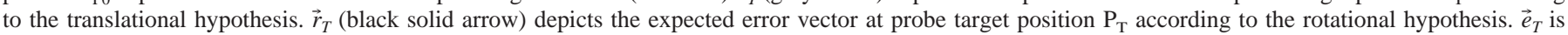

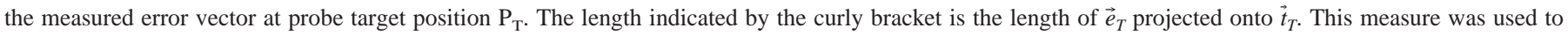
quantify the amount of generalization at the different probe targets (see METHODS). $\delta_{T}$ is the angular difference between P0 and $\mathrm{P}_{\mathrm{T}}$. 
effects. Eye fixation and saccade targets had to be continuously fixated within a tolerance window of $2.5 \mathrm{~cm}\left(3.6^{\circ} \mathrm{VA}\right)$ and $3 \mathrm{~cm}\left(4.3^{\circ} \mathrm{VA}\right)$ radius, respectively. Eye fixation breaks or release of the hand fixation stimulus immediately aborted the trial. Correct trials were defined as trials without fixation breaks and in which subjects reached to the target position within the time limits and within the allowed spatial tolerance window. Only correct trials were used for further analysis.

\section{Behavior Analysis}

Time course of adaptation. We used nonlinear least square regression to fit the directional reach errors ( $d e v)$ during the exposure and postexposure with an exponential function over successive trials $t$ to quantify incremental changes in the reach endpoint positions due to adaptation or readaptation (Fig. 2):

$$
\operatorname{dev}(\mathrm{t})=\text { offset }+\mathrm{ae}^{-\varepsilon \mathrm{t}}
$$

where $\varepsilon$ defines the learning rate, offset defines the asymptotic reach error and $a$ defines the scale. Directional reach errors were defined as the angular deviation of the direction of the reach from the direction of the target. Directions of the reach were measured as net direction of the whole movement, i.e., direction from the fixation stimulus to the target/reach endpoint. To compensate idiosyncratic biases, which were independent of adaptation, we subtracted the mean directional reach error in the preexposure period from the directional reach error in each trial for each individual subject before averaging data across subjects.

The exponential fit as measure of directional error was only used for demonstrating the incremental improvement during exposure and the negative aftereffects during postexposure. It was not intended to precisely analyze the time course of adaptation, where it has been suggested that double-exponential fits provide a better match (e.g., Krakauer et al. 2000), nor was it further used to quantify generalization. All further quantifications of reach error considered the spatial generalization pattern.

Quantifying generalization and its asymmetry. We first tested how adaptation transferred to untrained probe targets (generalization). To do so, we quantified the difference in reach endpoints to the different probe targets during baseline performance in the control condition and during the adapted behavior in the generalization period. Two alternative hypotheses concerning the spatial generalization pattern were tested. According to the translation hypothesis, the direction of the error vector is independent of the probe target position (light gray arrow in Fig. 1E). Any spatial profile of the generalization pattern across the workspace would affect only the length of the error vector, not its direction. Such generalization would suggest a transfer of the two-dimensional (2D) Cartesian endpoint position due to adaptation. According to the rotation hypothesis, the error vector is always tangential to the probe target direction (dark gray arrow, Fig. $1 E$ ). The generalization pattern across the workspace would affect the length and direction of the error vector simultaneously in a specific way. Such generalization would suggest a transfer of only the one-dimensional (1D) center-out direction of the adapted reach endpoints. Since we only adapt a single target direction in our experiment, the jump can be interpreted as either a positional shift or a directional change of the target; hence either generalization pattern is equally plausible.

The measured error vector at the adapted target in individual subjects might not perfectly match the physical displacement of the target (neither in size nor direction of the jump). We accounted for interindividual differences in adaptation when testing for the alternative translation and rotation hypotheses. We used the error vector at the adapted target (Fig. $1 E ; \vec{e}_{\mathrm{P} 0}$ ) as reference for predicting the error vectors at the probe targets according to the translation and the rotation hypotheses. The predicted error vectors for the translation hypothesis $\left(\vec{t}_{T}\right.$, gray arrow) and for the rotation hypothesis $\left(\vec{r}_{T}\right.$, black arrow) at each probe target $T$ were defined as:

$$
\begin{gathered}
\vec{t}_{T}=\vec{e}_{\mathrm{PO}} \\
\vec{r}_{T}=R\left(\delta_{T}\right) \vec{e}_{\mathrm{PO}}=R\left(\delta_{T}\right) \vec{t}_{T}
\end{gathered}
$$

where $R$ is a 2D rotation matrix, and $\delta_{T}$ equals the angular difference between the mean reach endpoint at $\mathrm{P} 0$ and the mean reach endpoint at the respective probe target in the control condition (Fig. 1E).

We compared the direction of the measured error vector at each target $\left(\vec{e}_{T}\right)$ with the vectors predicted by the two hypotheses. The residual errors, the deviations of the respective prediction and the empirical data, were computed as

$$
\begin{aligned}
\tau_{T} & =\left[\operatorname{angle}\left(\vec{e}_{T}\right)-\operatorname{angle}\left(\vec{t}_{T}\right)\right] / \delta_{T} \\
\rho_{T} & =\left[\operatorname{angle}\left(\vec{r}_{T}\right)-\operatorname{angle}\left(\vec{e}_{T}\right)\right] / \delta_{T}
\end{aligned}
$$

for the translation and rotation hypothesis, respectively. Note that $\delta_{T}$ denotes the maximal angular deviation that the predictions according to the two hypotheses would differ as a function of probe target direction. The further away from $T 0$ a probe target is, the larger is $\delta_{T}$. Therefore, the residual errors were normalized by $\delta_{T}$ to account for the expected variable amount of deviation for the different probe targets and to be able to compare the residual error across different probe target position. The residual error will be close to 0 if the data fit the tested hypothesis and close to 1 if the data fit the alternative hypothesis.

Testing the translation against the rotation hypothesis requires an effect of adaptation at the respective probe target in the respective subject. If endpoints do not significantly deviate from the target position, the direction of the error vector, and hence the residual
Fig. 2. Learning curves during the generalization task. The average directional reach errors (mean \pm SE, baseline corrected) across $N=16$ and $N=13$ subjects are shown for $\mathrm{CCW}(A)$ and $\mathrm{CW}(B)$ adaptation and fitted with an exponential function (gray curves) in exposure and postexposure periods. The data points during the probe period reflect directional reach error $( \pm \mathrm{SE})$ for the target jump trials (refresher trials) presented during this period. The refresher trial appeared only in a fraction of the trials and randomly so for each subject. Therefore, for each trial, only a fraction of the subjects contributed to the respective average data point, explaining the larger variance across trials.
A

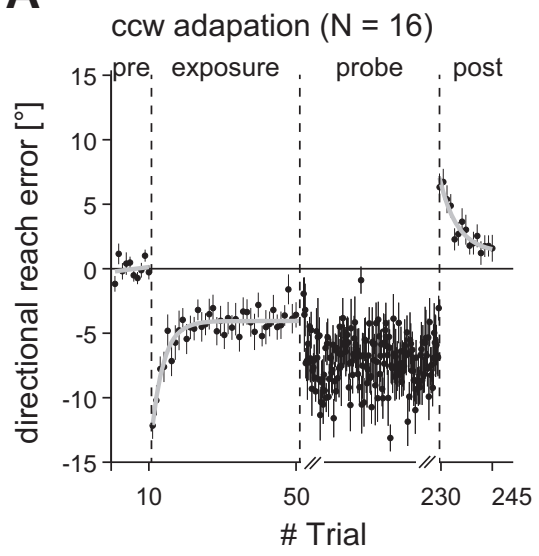

B

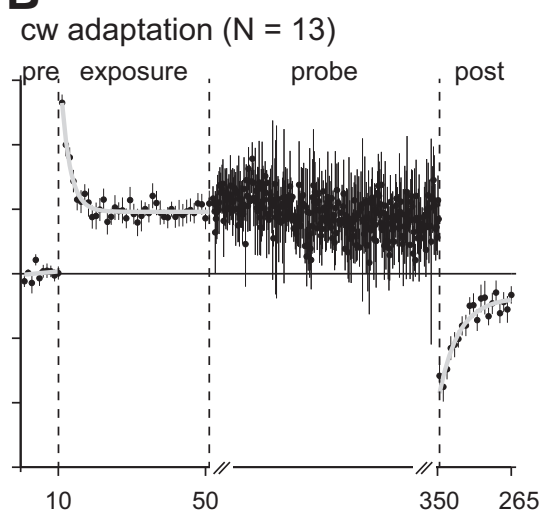


errors, becomes random. This would likely affect especially those probe targets which have a large distance from the adapted target. Therefore, we computed the average residual errors across subjects at each probe target position only for subjects in whom reach directions in the control and test conditions were significantly different (ranksum test).

We found that the direction of this error vector was independent of the probe target position (Fig. 3, $A-C$ ), supporting the translation hypothesis. This will be described in more detail in RESULTS, but we used this knowledge to then quantify the amount of adaptation at each probe target by projecting the measured error vector $\left(e_{T}\right)$ onto the direction of the predicted error vector for the translation hypothesis $\left(\vec{t}_{T}\right)$. This means, the relative adaptation (RA) for each probe target $T$ was defined as scalar value:

$$
\mathrm{RA}=\frac{\left\langle\vec{e}_{T} \cdot \vec{t}_{T}\right\rangle}{\left|\vec{t}_{T}\right|}=\frac{\left|\vec{e}_{T}\right|}{\left|\vec{t}_{T}\right|} \cos \left[\operatorname{angle}\left(\vec{e}_{T}\right)-\operatorname{angle}\left(\vec{t}_{T}\right)\right]
$$

with $<\cdot>$ being the dot product. This procedure measures the transfer of the translational reach error to probe targets, not the directional reach error (Fig. $1 E$ ). Note that the results on the symmetry/asymmetry of generalization as a function of probe target position (see RESULTS) per se did not depend on this optimized quantification of the adaptation transfer. The results held equally true when symmetry/ asymmetry was tested simply with the absolute size of the directional reach error (data not shown).

The resulting generalization function $\operatorname{RA}(T)$ was fitted with a skewed $t$-distribution (Azzalini and Capitanio 2003), which is parameterized by five parameters: $\{\mu, \sigma, \lambda, \alpha, \mathrm{df}\} . \mu$ defines the center of the distribution (which is not the mean), $\sigma$ defines the width, $\lambda$ defines the skewness, $\alpha$ is a scaling factor, and $\mathrm{df}$ is the degrees of freedom (which determines the kurtosis). This distribution can be fit to any unimodal distribution whose first four moments are arbitrary, provided the excess kurtosis is positive. We quantified the asymmetry of the generalization function using the parameter $\lambda$ from this fit, i.e., its skewness. This means asymmetry in our case is more than a mere shift of the distribution relative to a reference point (Gonzalez Castro et al. 2011). Instead, it measures lack of symmetry with respect to any reference. This function was fit to the behavioral data of all subjects on a least-mean-squares basis (Matlab Curve fitting toolbox).

Only correct trials were included in the analysis. Yet, adaptation might occur in error trials that nevertheless provided feedback to the subjects. For example, error trials included trials with fixation breaks during the target hold period and trials with target acquisition slower than the allowed threshold. As a control, we repeated our analyses including such trials and saw no change in the pattern of results (data not shown).

Exclusion of subject. Subjects who faced difficulties in task performance were excluded from analysis. First, subjects with a success rate of $<40 \%$ (not counting eye fixation breaks during fixation period) in the initial test condition were excluded from the analysis (1 subject each in the CW and CCW experiment). Second, subjects who did not show significant adaptation at T1 during the generalization period (reach error at T1 not significant larger than zero, Wilcoxon signedrank test) were not included in the analysis, since neither of the analyses in this study could be performed on their data ( 2 in CCW, 2 in $\mathrm{CW}$ ). The high fraction of subjects showing adaptation made us confident that in general the saccadic suppression worked in masking the target jump. However, for the most conservative approach, subjects who reported that they had at least in some trials noticed the target jump were treated separately in the analysis (10 in CCW, 3 in $\mathrm{CW})$. Unless mentioned otherwise, the figures in the paper do not contain these subjects, but instead as a control, our analyses were
A

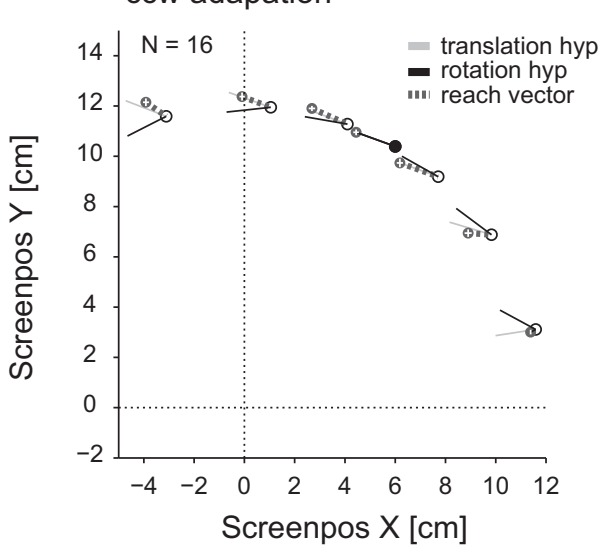

C

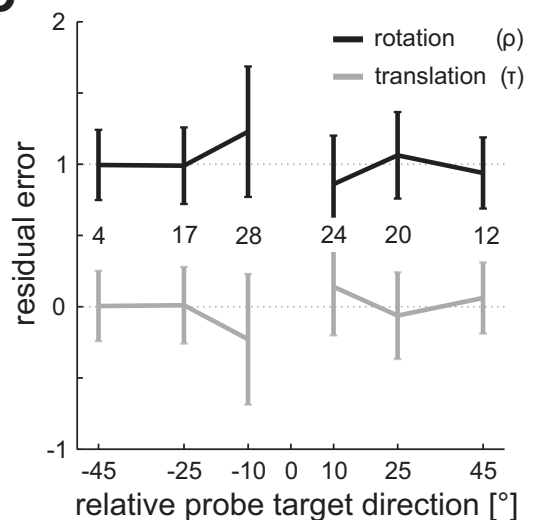

B

Cw adaptation

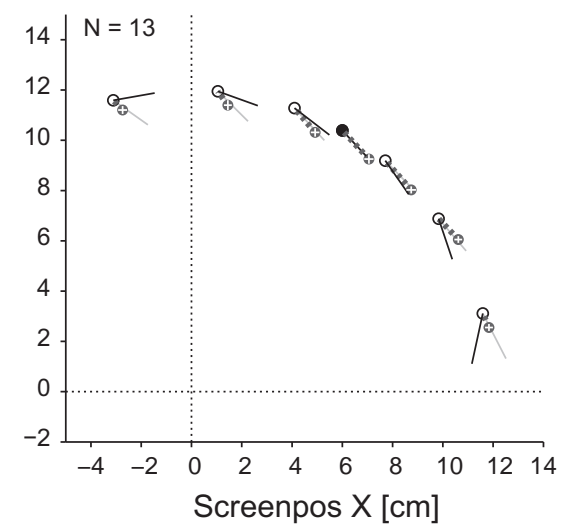

D

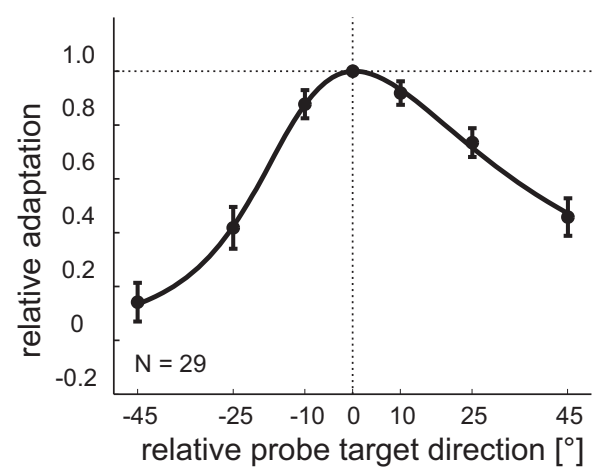

Fig. 3. Generalization of adaptation. A: average reach endpoints (gray circles) and resulting reach error vectors (dotted lines) in the $\mathrm{CCW}$ experiment. For comparison, the expected error vectors for the translational (light gray) and rotational (black) hypotheses (hyp) are shown. Open circles depict probe target positions. The black circle marks T1. Reach endpoints for each subject are baseline corrected for their reach positions in the control task. $B$ : same as $A$ for the $\mathrm{CW}$ experiment. $C$ : translational vs. rotational generalization. Residual errors are shown for the rotation $\left(\rho_{T}\right.$, dark gray) and translation $\left(\tau_{T}\right.$, light gray) hypothesis at each probe target. Data were pooled across the $\mathrm{CW}$ and $\mathrm{CCW}$ experiments and across subjects. Probe target positions ( $x$-axis) are labeled relative to the T1/P0 at $60^{\circ}$. Positive deviations are defined as probe targets into the direction of the target jump; negative deviations are probe targets in the direction opposite to the jump. At each probe target, only those subjects were included who showed a significant difference in direction between the test and the control condition (see nos. within figure). $D$ : asymmetry of generalization. Average relative adaptation (mean $\pm \mathrm{SE}$ ) for data pooled across the CW and $\mathrm{CCW}$ experiments and across subject and fitted with a skewed $t$-distribution. Probe target positions is as in $C$. 
repeated separately for these subjects, and respective results are reported separately (see RESULTS). The most conservatively reduced dataset consisted of $16(\mathrm{CCW})$ plus $13(\mathrm{CW})$ subjects and was used for all main analyses.

\section{Model Simulations}

Neural network model. We used a three-layer recurrent artificial neural network to simulate our target-jump adaptation experiment. The idea was to test changes of the neuronal representations in the network as a result of local target jump adaptation, mimicking the human experiment. The model was a learning model which was first trained to perform "standard reaches," i.e., the network learned a congruent 1-to-1 mapping between sensory input and motor output across the full workspace. Target jump adaptation was then implemented in a second step by retraining the network to cope with a locally perturbed input-output mapping (details see below). This means that only the locally perturbed mapping at the location of the primary target was trained to the network during this adaptation phase. Finally, generalization to unperturbed probe targets was tested. Importantly, no constraints on the spatial profile of generalization were imposed on the model. The supervised learning in the model was driven by only the target error signal, namely the difference between the actual motor layer output and the desired motor layer output. We thereby could test the generalization profile as a result of the local adaptation, like in the human experiment.

The present model was nearly identical to a model that had been developed in a previous study to perform spatial sensorimotor mappings in a context-specific manner. Here we will sketch the concept of this previous model briefly, while details of the implementation can be found in the original publication (Brozovic et al. 2007). We will then explain modifications to the previous model that were necessary for simulating the current behavioral experiment. The previous and current model consisted of three layers: a visual input layer, a recurrent hidden layer and a motor output layer. All layers are fully connected via feedforward connections plus an optional additional feedback projection from the motor layer to the hidden layer. The visual input layer is a priori topographically organized and represents the taskrelevant workspace, which could either be the $360^{\circ}$ circular space of center-out reach directions or the 2D Cartesian space of reach endpoints in the fronto-parallel plane. Input layer neurons had Gaussian tuning profiles and tiled the workspace evenly. Output layer neurons represented the same workspace as the input layer but initially had no spatial tuning. They developed Gaussian tuning profiles as a result of learning a standard 1-to-1 mapping from input to output. In this first training step the network was trained from an initially network-wide random connectivity to a state in which it produced a population tuning with a Gaussian profile in the output layer in response to a local input to the input layer at the corresponding position of the workspace (see below). The intermediate (hidden) layer with recurrent connections received direct information from the input layer and was optionally reciprocally connected with the output layer (see Fig. $5 A$; Network II in Brozovic et al. 2007). All connection weights were updated according to a supervised backpropagation-through-time learning algorithm. The previous network was trained to conduct four different spatial sensory-to-motor mappings, depending on the four possible levels of explicit context input. The detailed implementation of the network and algorithm can be found in Brozovic et al. (2007).

Two modifications of the original model were made to simulate the current experiment. First, and most importantly, we trained the network to conduct only one global and congruent mapping rule, which was then locally perturbed. We provided no context input to the model to distinguish between different valid mappings. Especially, no additional input existed that would have provided explicit information about the exposure or nonexposure to the target jump. The lack of explicit input about the exposure to target jumps mimics the consequences of the saccadic suppression in our experiment: subjects did not explicitly know about the perturbation trials. The only source of information for driving learning in this scenario is the target error at the end of the trial (see Simulation procedure below for details).

Second, for better comparison with the human data, we used a 2D workspace, while the original model was 1D. Note, the network architecture and the learning algorithm are mathematically identical for the $1 \mathrm{D}$ and $2 \mathrm{D}$ model. The spatial selectivity profiles of the input layer units $R_{i}(x, y)$ changed to the following $2 \mathrm{D}$ definition:

$$
R_{i}=\exp \left[-\left(\frac{x-x 0_{i}^{i n}}{\sigma_{x}^{i n}}\right)^{2}-\left(\frac{y-y 0_{i}^{i n}}{\sigma_{x}^{i n}}\right)^{2}\right]
$$

where $x 0_{i}^{i n}$ and $y 0_{i}^{i n}$ define the receptive field center, and $\sigma_{x}^{i n}$ and $\sigma_{y}^{i n}$ the tuning width of the respective input unit. $x$ and $y$ define the positions of the target.

The desired activation of the output unit (teaching signal) $\mathrm{T}_{i}$ was defined as:

$$
T_{i}=\exp \left[-\left(\frac{x-x 0_{i}^{\text {out }}+\omega_{x}}{\sigma_{x}^{\text {out }}}\right)^{2}-\left(\frac{y-y 0_{i}^{\text {out }}+\omega_{y}}{\sigma_{x}^{\text {out }}}\right)^{2}\right]
$$

The "jump" parameters $\omega_{x}$ and $\omega_{y}$ allow training a spatial offset between visual input and motor output. In the previous implementation of the model, the 1D equivalent was used for a global rotation of the input-output relationship, e.g., a $90 \mathrm{CW}$ rotation of all targets relative to the visual instruction. In the current implementation, we used the offset only at a single visual input to simulate a target jump from a primary target $\mathrm{T} 1$ to a jumped target position $\mathrm{T} 2$. This means the teaching signal was such that, after the network had learned the target jump perfectly, in response to a cue at the primary target position T1 it would produce a Gaussian shaped population response in the output layer, which was centered on the jumped target position T2. During the exposure phase (second learning step, see below), the jump vector was set to correspond to an offset of the center-out reach direction of $15^{\circ}$ at an eccentricity of eight units. It was set to zero outside the exposure phase. The centers of the output units $x 0_{i}^{\text {out }}$ and $y 0_{i}^{\text {out }}$ were the same as for the input units.

As an additional minor change compared with the original Brozovic model, the number of hidden layer units was set to 350 to take the increased number of spatial inputs into account.

Neural network parameters. The neural network model simulated a square workspace of visual input and motor output. The center of the input and of the output units $x 0_{i}^{\text {in/out }}$ and $y 0_{i}^{\text {in/out }}$ were equally spaced from -14 to +18 arbitrary units, in intervals of 2 units (289 input/ output units). The additional workspace of 4 units in the positive ranges (from +14 to +18 to the right and to the top) was added to avoid margin effects in the surrounding region of the adapted position, which was at $(+8,+8)$ units. The tuning widths $\sigma_{x}^{i n}$ and $\sigma_{y}^{i n}$ of the input layer units were set to 4 units.

The tuning widths of the output units, $\sigma_{x}^{\text {out }}$ and $\sigma_{y}^{\text {out }}$, were set to 6 units. We used the center of mass to quantify the model's motor output, i.e., the "reach" position was decoded via the sum of vectors defined by the a priori assigned preferred position of each output neuron weighted with its activation level [population vector decoding (Georgopoulos et al. 1986)].

The arrangement of target positions mimicked a center-out task across the full circle with varying eccentricities. We presented targets at 936 different positions within an annulus ranging from 2 to 14 units eccentricity. Radial spacing of the target positions was 1 unit; angular spacing $5^{\circ}$.

Simulation procedure. We trained the model in the following steps to simulate the target jump adaptation paradigm.

STANDARD MAPPING. Starting from a random-weights initialization, the single-mapping network was trained to perform a 1-to-1 mapping of the visual input to a congruent motor output across the workspace. This means, if a visual target was presented at position $(x 0, y 0)$, it created a local activation profile centered on this position 
in the input layer, and the network was trained to produces a local activation profile centered at the same position in the output layer. The idea of the standard mapping learning procedure is that, after learning, the network can perform reaches across the workspace, as is the case for human subjects when they start the experiment. After learning, standard mapping trials mimic standard trials of our behavioral experiment. Training was continued until the mean square error between the desired and actual activity profile across output layer neurons and across contexts dropped below 0.0001 .

TARGET JUMP. Starting from the training state in which they reliably performed the standard mappings, the model then was trained to perform a mapping from a single input position (T1) to a target output position which was off-set relative to the standard mapping (T2). This mimicked the target-jump trials in the exposure phase of our current behavioral experiments. The training algorithm for the implementations of the target jump adaptation was identical to the algorithm during initial learning of the standard mapping.

GENERALIZATION. After the model was adapted to the target jump, the synaptic weights were kept fixed to mimic probe trials without feedback, i.e., without an error signal. We then probed the model across the workspace to test how adaptation at one location generalizes to other untrained targets, i.e., to assess postadaptation generalization. Generalization was tested as a function of reach direction, as in our current behavioral experiment.

We ran all simulations with 25 different sets of random initialization of the weights. The 25 seeds in the analysis of the model data were treated equivalently to the different subjects of the experimental data. Yet the across-seed variance of the simulation data was not intended to mimic the across-subject variance in the human data.

Model-neuron tuning analyses. The response field (RF) position of an output or hidden layer neuron was defined as the position of the spatial inputs which gave the maximum activity of the respective neuron. RF size was defined as the number of spatial input positions, which gave an activity level larger than the half-maximum activity. RF amplitude was defined as the maximum activity of the unit. Units with an activation level less than 0.05 were considered "inactive" and excluded from the analysis. Changes in RF size and RF amplitude were quantified with a modulation index:

$$
\Delta \text { Parameter }=\frac{\left(\text { Parameter }_{\text {Generalization }}-\text { Parameter }_{\text {Standard }}\right)}{\left(\text { Parameter }_{\text {Generalization }}+\text { Parameter }_{\text {Standard }}\right)}
$$

Changes in RF position were analyzed as difference:

$$
\Delta \text { RFposition }=\text { RFposition }_{\text {Generalization }}-\text { RFposition }_{\text {Standard }}
$$

\section{RESULTS}

Subjects followed the instructions and conducted the saccade and reach simultaneously. There were no significant differences in reaction time between different experimental phases. In the generalization task, the reach reaction times on average across subjects were $226 \pm 29 \mathrm{~ms}$ (mean \pm standard deviation), and the saccadic reaction times $215 \pm 24 \mathrm{~ms}$. In the control task, reach reaction times were $239 \pm 34 \mathrm{~ms}$ and the saccadic reaction times $225 \pm 32 \mathrm{~ms}$. We compared saccade parameters in the control and generalization task to make sure they do not vary between the two task conditions. The mean variances of saccade endpoints (endpoint accuracy) were $0.39 \pm 0.14 \mathrm{~cm}$ and $0.23 \pm 0.09 \mathrm{~cm}$ during generalization, and $0.29 \pm 0.07 \mathrm{~cm}$ and $0.14 \pm 0.03 \mathrm{~cm}$ during control for the two saccade targets, respectively. These differences were not significant $(P=$ $0.45 / 0.33$, paired $t$-test). The initial saccade directions (at $3 \mathrm{~cm}$ eccentricity) toward the two saccade targets (at $210^{\circ}$ and $270^{\circ}$ ) were $204 \pm 2^{\circ}$ and $262 \pm 1.7^{\circ}$ during generalization and $208 \pm 2^{\circ}$ and $262 \pm 1.9^{\circ}$ during the control trials. These differences were not significant $[P=0.24$ and 0.8 ; nonparametric test for the equality of circular means (Fisher 1993)].

\section{Target Jump Adaptation}

Our target jump paradigm reliably induced reach adaptation. This is shown by an incremental reduction of the reach error during exposure and the negative aftereffect in the postexposure period (Fig. 2 and Table 1). The learning rates in the exposure were $-0.33^{\circ}$ and $-0.43^{\circ}$ per trial, and the initial directional reach errors in the postexposure were $7.0^{\circ}$ and $9.8^{\circ}$ for the $\mathrm{CCW}$ and $\mathrm{CW}$ experiments, respectively. All values are significantly different from zero (95\% confidence limits, see Table 1). Adaptation was incomplete, and the size of the initial reach error during postexposure approximately matched the difference between the initial reach error during exposure and the asymptotic remaining reach error ("offset") at the end of the exposure period. This difference is also the level of adaptation that was sustained during the generalization period.

Since the results from the $\mathrm{CCW}$ and $\mathrm{CW}$ generalization were not qualitatively different, we merged the data of both experiments in a mirror-symmetric fashion and present it jointly in the following.

As a control experiment, we tried to induce reach adaptation with a target jump paradigm, but without the accompanying saccade. All other parameters were kept equal. In this case, subjects $(N=4)$ show neither incremental improvement, nor a negative aftereffect. Reach endpoints were only significantly different from the displaced target position in the first trial after beginning of the exposure period or the first trial after beginning of the postexposure, but then performance immediately returned to baseline in the second trials (data not shown). Since these results are consistent with previous studies (Diedrichsen et al. 2005), we did not further investigate this condition. This control confirms the existing view that a consistent and noticeable target jump due to its predictability leads to an explicit

Table 1. Initial directional reach error, learning rate, and asymptotic reach error (offset) as derived from an exponential fit for the

\begin{tabular}{|c|c|c|c|c|c|c|}
\hline & \multicolumn{2}{|c|}{ Initial Reach Error, ${ }^{\circ}$} & \multicolumn{2}{|c|}{ Learning Rate, ${ }^{\circ}$ trial } & \multicolumn{2}{|c|}{ Offset, ${ }^{\circ}$} \\
\hline \multicolumn{7}{|l|}{$\mathrm{CCW}$} \\
\hline Exposure & -12.3 & $(-10.7-13.6)$ & -0.33 & $(-0.22-0.43)$ & -4.1 & $(-3.7-4.4)$ \\
\hline \multicolumn{7}{|l|}{$\mathrm{CW}$} \\
\hline Exposure & 13.2 & (11.1 15.7) & -0.43 & $(-0.34-0.51)$ & 4.8 & $(4.65 .0)$ \\
\hline Postexposure & -9.3 & $(-7.3-11.0)$ & -0.19 & $(-0.10-0.28)$ & -1.8 & $(-0.9-2.8)$ \\
\hline
\end{tabular}
exposure and postexposure periods in the $C C W$ and $C W$ experiment

Values in parentheses denote $95 \%$ confidence intervals. Target jump size was $15^{\circ}$. CW, clockwise; CCW, counterclockwise. 
"strategic" updating of the intended reach goal, rather than an implicit adaptation, in otherwise identical task conditions.

In summary, the incremental improvement in reach performance and significant negative aftereffects confirm that reach adaptation was induced by our task, which induced target errors, but no sensory prediction errors about hand position or cross-modal mismatch about hand position during the movement.

\section{Translational Generalization}

We tested whether adaptation transferred to untrained probe targets with a fixed 2D offset in the Cartesian endpoint position (translational hypothesis) or a 1D offset in the center-out direction (rotational hypothesis). Figure $3, A$ and $B$, shows the average reach endpoints across subjects and the resulting reach error vectors (from target position to reach endpoint). Across the population of subjects (Fig. 3, $A-C$ ), the generalization pattern fits the translation hypothesis and rejects the rotation hypothesis. The average residual error (i.e., the measure of how much the data points differ from the predictions of the respective hypothesis) for the translation hypothesis across all subject and all targets is -0.032 , which is not significantly different from $0(P=0.84, t$-test $)$. The average residual error for the rotation hypothesis is 1.032 , which is significantly larger than $0\left(P<10^{-7}, t\right.$-test $)$. Note that values around zero for one hypothesis automatically lead to values around 1 for the other hypothesis. But the fact that the average residual error for the rotation hypothesis is significantly larger than 0 contains the important information that the nonsignificant result of the translation hypothesis is not due to a lack of statistical power.

\section{Range and Asymmetry of Generalization}

Figure 3, $A$ and $B$, shows that average reach endpoints in the adapted condition were shifted into the direction of the target jump, especially for probe targets neighboring T1. There was less amount of shift of the reach endpoints, i.e., less generalization effect, for probe targets further away from T1. For both datasets, the generalization effect was stronger for probe targets in the direction of the target jump [CCW from T1 for CCW adaptation (Fig. $3 A$ ) and $\mathrm{CW}$ from $\mathrm{T} 1 \mathrm{for} \mathrm{CW}$ adaptation (Fig. $3 B$ )], compared with probe targets on the opposite side. We quantified the amount of transfer of the reach error as a function of distance from T1. As already visible in the separate datasets (Fig. 3, $A$ and $B$ ), the relative adaptation (length of the reach error vector compared with the reach error at T1) decreased with increasing distance from $\mathrm{T} 1$ across subjects (CW and CCW dataset combined, Fig. 3D). This resulted in significant differences in the amount of adaptation for the different target positions $\left[P<10^{-9}, F_{0.05}(6,168)=29.1, N=\right.$ 29, repeated-measures ANOVA].

Besides the drop-off of the reach errors with distance, the generalization showed an asymmetry. Probe targets in the direction of the jump (defined as positive relative target directions) showed larger relative adaptation than probe targets in the direction opposite to the jump (defined as negative relative target directions). We quantified this asymmetry by fitting a skew $t$-distribution to the subject generalization data (Fig. 3D). The fit gave a positive $\lambda$ of 1.88 [95\% confidence interval (CI) (0.23 3.53)] indicating a rightward skewness. This rightward skewness indicates a greater adaptation effect for probe targets in the direction of the jump as opposed to probe targets in the direction opposite the jump.

The generalization results show that, in our target jump experiment, the adaptation generalizes in a translational fashion, over a limited spatial range, and asymmetrically more strongly toward the side of the perturbation.

\section{Subjects Reporting Occasional Target Jump Visibility}

The main results of human psychophysics contain only data from subjects who reported not to have seen the target jump (see METHODS). As a control, we also analyzed the subpopulation of subjects who reported to occasionally having seen the target jump. Within this group, adaptation to target jump was as common as in the main group of subjects who did not notice the jump at all. The fraction of subjects who did show significant adaptation within the group with only partial suppression of target jump visibility was 13 out of 15 , and was not smaller than the fraction in the group of subjects who reported complete suppression of target jump visibility (29 out of $32, P=0.92$, Pearson's $\chi^{2}$ test with Yates' correction for continuity). Since we did not probe and quantify individual subject's ability to detect jumps on a graded scale, we were not able to correlate the degree of subjective suppression of the jump with the degree of adaptation. However, none of the subjects reported having seen a jump over several successive trials, which is probably the reason why the general adaptation effects were not abolished. In the partially aware group, we found a trend toward a skewed generalization pattern in the same direction as for the main group of subjects, but the skewness was not significant $[\lambda=1.73$, CI $(-1.534 .99), N=13]$. This lack of significance compared with the main group seems to be mainly a lack of statistical power. When we randomly subsampled $(\times 500)$ the data in the main group to match the number of subjects in the partially aware group, then in $\sim 90 \%$ of the subsamples the skewness was not significant either.

\section{Target-Jump Adaptation in Neural Network Simulations}

We used a recurrent multilayer neuronal network model (Fig. 4A) to simulate the adaptation learning in our task. After training the network to the local target jump, we investigated the resulting generalization pattern and the associated neural tuning changes in the underlying neuronal network (see METHODS). The idea of this simulation was to find out if asymmetric generalization could emerge from simple network structures without build-in asymmetric constraints, i.e., just from the spatial layout of the task, and, if so, what neural tuning changes would occur and be necessary as a prerequisite for such adaptation.

A multilayer neural network as used here has enough degrees of freedom to adjust its input-output relationship to any required mapping. Correspondingly, like the network could learn its initial 1-to-1 mapping of visual input space onto motor output space from a naive state with random mapping (see METHODS); it could quickly learn to compensate a local perturbation of this structured 1-to-1 map according to the target jump protocol. In other words, the model learned to associate the spatial cue at the $\mathrm{T} 1$ position with a motor output to the T2 rather than T1 position. The nontrivial question was how this 
A

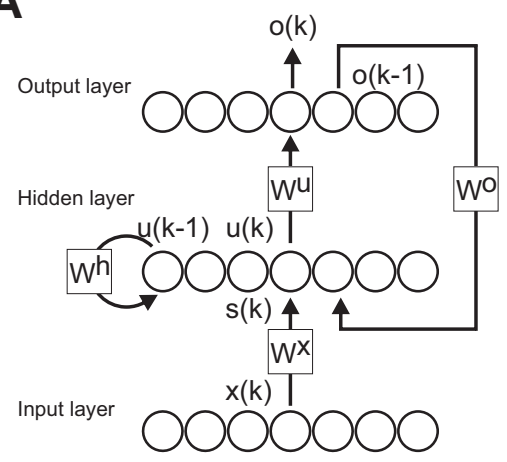

B

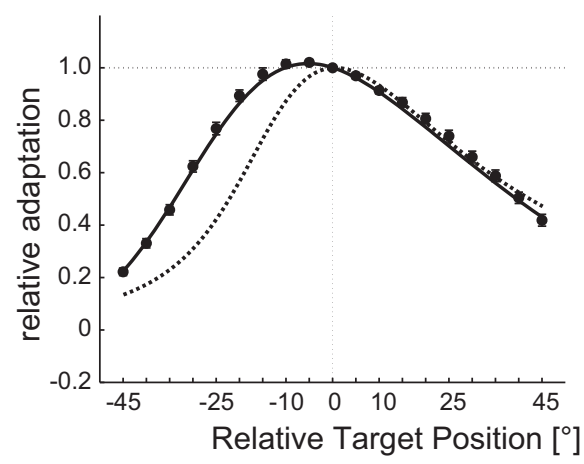

C

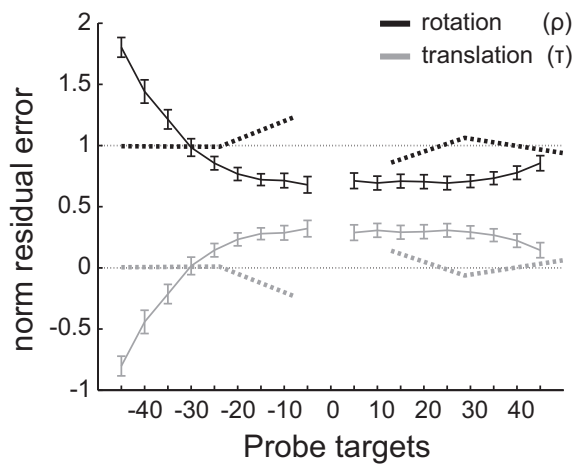

D

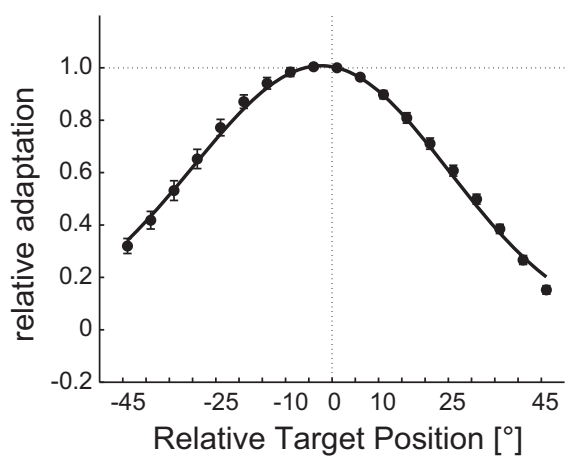

Fig. 4. Simulation of target jump adaptation with a recurrent neural network model. A: network architecture. The input (sensory) layer encodes the position of the visual stimulus. The hidden layer receives direct input from the sensory layer and input via feedback connection from the output layer. Additionally, the hidden layer units are recurrently connected. The total input into the hidden layer is defined as $s(k)=W^{k} x(k)+W^{h} u(k-1)+$ $W^{O} o(k-1)$, with $W$ defining weight matrices, and $k$ defining time steps. The output of the hidden layer is defined as $u(k)=\phi[s(k)]$, with $\phi$ being a sigmoid transfer function. The output (motor) layer receives the hidden layer activation as input $o(k)=\phi\left[W^{u} u(\mathrm{k})\right]$. Reach direction is decoded with a population vector based on the output layer activities. For details see (Brozovic et al. 2007) and METHODS. $B$ : asymmetric generalization curve of the feedback version of the model. Data points show the average relative adaptation (mean $\pm \mathrm{SE}$ ) over 25 model simulations with independent random initialization. Conventions are the same as in Fig. 3D. Dotted line depicts the fitted generalization curve of the human psychophysical experiment (Fig. $3 D$ ) for comparison. $C$ : average residual error for rotation vs. offset hypothesis for the same 25 stimulations of the model. Other conventions are the same as in Fig. 3C. Dotted line shows the results of the human psychophysics experiment (Fig. $3 C$ ). D: same as in $B$, but for 22 simulations of the feedforward variation of the model in which fixed tuning properties in the hidden layer were enforced (see main text). Here positive skewness of the generalization curve is lacking. local adaptation would generalize to unlearned probe targets. Like in the human subjects, the amount of transfer of adaptation to nonexposed probe targets (tested at the corresponding positions as for the human data) significantly decreased with increasing distance from T1 $\left[P<10^{-10}, F_{0.05}(18,432)=155\right.$, $N=25$, repeated-measurement ANOVA]. Most importantly, the model produced significant asymmetric generalization $[\lambda=$ 3.17, CI (2.79 3.55)]. Even though the model data are not perfectly matching the human data, the skewness of the model data is within the range of the confidence limits of the human experimental data (Fig. 4B).

Additional to the asymmetric generalization, we established that the model's motor output in generalization trials was consistent with the second aspect of human behavior, the translational generalization. We found that the output of the model better matched the translation hypothesis than the rotation hypothesis (Fig. 4C). The average residual error for the translation hypothesis across the 25 random initializations and across all targets was 0.12 , which was close to 0 , even though significantly different from it $\left(P<10^{-8}, t\right.$-test). For the rotation hypothesis, the average residual error was $0.88(P<$ $10^{-20}, t$-test), which is close to 1 and, importantly, larger than the residual error for the translation hypothesis $\left(P<10^{-12}\right.$, $t$-test). In contrast with the human data, the residual errors for the two hypotheses systematically deviate more strongly for probe targets that are further away from the adapted target.

In summary, our model simulates the human behavioral data qualitatively in terms of asymmetry, spatial range, and translational frame of reference of generalization. It should be emphasized that the model was not trained to produce either of these effects.

\section{Model Tuning Changes During Adaptation}

Previous computational studies simulated typical visuomotor adaptation data with symmetric generalization. These previous results suggested that the tuning in visuomotor areas ("hidden" layer of a multilayer network) should not change during visuomotor rotation paradigms, since this was the requirement for generalization to be symmetric in the model (Tanaka et al. 2009). We tested this prediction by fixing the tuning properties of the hidden layer in our model and could confirm the observation. For this test, we ran a model simulation in which the weights from the input to the hidden layer ( $W^{X}=$ constant, Fig. $4 A$ ), as well as the weights of the recurrent connections within the hidden layer $\left(W^{h}=\right.$ constant $)$ were fixed after learning the standard mapping. Hence, no further learning of these connections during the adaptation phase was allowed. Additionally, the network architecture was changed such that the feedback connections from output to hidden layer were removed during all phases $\left(\mathrm{W}^{\mathrm{O}}=0\right)$. All three measures in combination were necessary to keep the tuning in the hidden layer constant. This modified model was still able to learn the target jump, but the generalization pattern no longer showed a significant skewness $[\lambda=-0.63$, CI $(-1.550 .28)$ ] (Fig. 4D). This simulation suggests that changes of the tuning properties in the hidden layer of the model (putative visuomotor structures of the brain) are critical for asymmetric generalization with a positive skewness in such a network.

To see if we could derive testable predictions for neurophysiology from the model, we analyzed the tuning changes of units in the hidden and the output layer of the network. Tuning changes were quantified as changes to the response field (RF) 
of the units. The RF is defined as the range of visual cue positions, which activate the respective unit. Figure $5 A$ plots the RF of an example output unit and an example hidden layer unit during the control and generalization task. To better illustrate the RF changes in the two units due to adaptation and quantify the changes as function of RF locations, we plot the RF contours of three examples units of the output layer and three example units of the hidden layer before and after
Fig. 5. Tuning changes (after/before adaptation) in hidden and output layer of the model due to adaptation to target jump. A: color-coded activity map (response fields) of a single example output unit (left column) and a single hidden unit (right column) during the control (top row) and generalization task (bottom row). The color map indicates the response of the respective neuron as a function of spatial cue position and is scaled to the peak activity of the neuron. $B$ : response field (RF) contours of 3 example units in the output layer (left panel) and 3 example units in the hidden layer (right panel) before (blue) and after (red) adaptation. Gen, generalization; Cnt, control. The contours outline the $50 \%$ activity level of each RF. The open circle indicates the T1 position; the solid black circle, the T2 position. The neurons with the dotted outlines are the same as shown in $A$. $C$ : adaptation-induced changes in the RF properties (left: position; middle: size; right: peak activity) of all output layer units. Average data over 25 independent random network initializations are shown separately for 289 units in the output layer. Top panels: the result for each neuron is plotted as a color-pixel at the position of the neurons RF center position prior to adaptation. Top left: the color indicates the amount a unit shifts its position in the $y$-direction due to adaptation. For example, the blueish color at the T2 position (solid black circle) indicates that an output neuron with a RF position at $\sim 7$ arbitrary units (AU) to the right and $2 \mathrm{AU}$ to the top shifts its RF center down by $\sim 3$ AUs during adaptation. Bottom left: the same changes in RF position along a circle with a radius matching target jump eccentricity (depicted in the top panel). Zero corresponds to the T1 position; the arrow indicates the T2 position. Middle column: change in RF size computed as modulation index (see METHODS); otherwise same conventions as in left column. Right column: change in RF peak activity as modulation index; otherwise same conventions as left column. $D$ : results for the 350 hidden layer units; same conventions as in $C$.
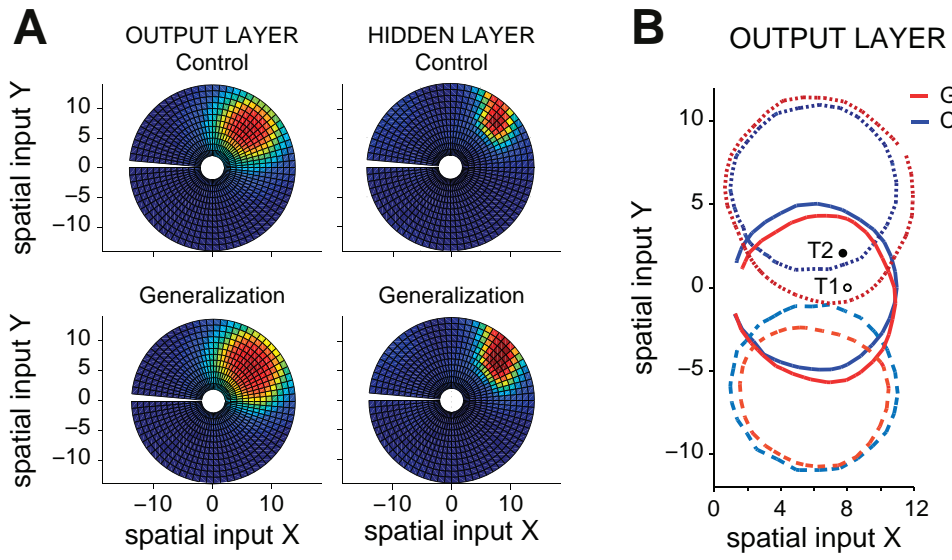

HIDDEN LAYER

\section{C output layer}
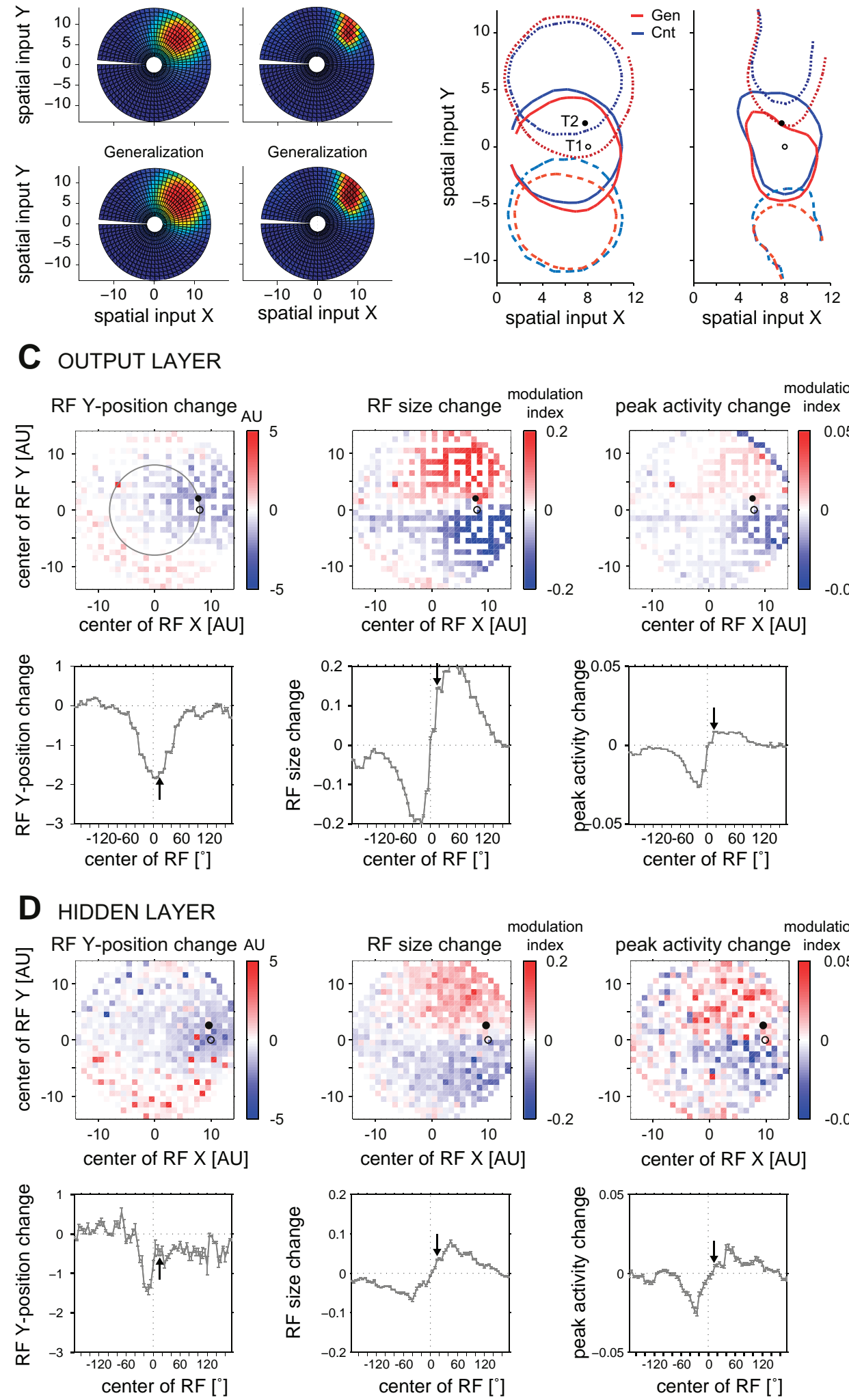

peak activity change index

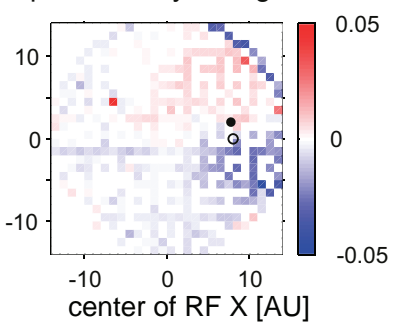


adaptation (Fig. 5B). The outline of spatial positions that elicit half-maximum activity for the respective unit is shown. The RF of the middle unit of the three chosen units in each layer overlaps the T1 location; the other two units cover neighboring locations, one in the direction of the target jump and one opposite to the target jump. In both the hidden and the output layer, the unit in the direction of the target jump increases its RF size. This does not happen by a concentric expansion of the RF boundaries, but the size increases especially at the RF flank facing the T1/T2 locations. In contrast, the unit opposite of the target jump direction decreases its RF size. This effect is again strongest at the flank of the RF facing the T1/T2 locations. These one-sided activity changes effectively also cause a shift of the center of the RF opposite to the direction of the target jump, whereas no systematic change of RF sizes or positions occur in the $x$-dimension. This is true for the units with RF locations in the direction of the target jump as well as for units with RF in the opposite direction. Figure 5, $C$ and $D$, shows changes in RF position in the $y$-dimension (left column), RF size (middle) and unit peak activity (right) for all output and hidden layer units due to adaptation. In the top rows, the units are arranged on the map according to their RF position before the adaptation. The bottom rows show RF changes for units with preferred position being located along a circle with the radius that matches the $\mathrm{T} 1$ eccentricity (marked in top panel of Fig. 5C). In summary, the units with RF locations in the direction of the target jump show an increase in RF size, whereas units with RF locations in the direction opposite of the target jump show a decrease in RF size. As a result, both units in and opposite to the direction of the target jump shift their RF center in a direction opposite to the target jump. Such a shift in the opposite direction of the target jump in single units results in an overall shift of the population output of these neurons in the direction of the target jump. In this way, an adapted reach to the T2 target position is achieved. Only units with RF location in the vicinity of the target jump location [within approximately $+70^{\circ}$ and $-40^{\circ}$ angular direction around $\mathrm{T} 1$ (T1 $=0^{\circ}$, i.e., positive $x$-axis in the model; see METHODS)] change their tuning properties. Further away from this location, the RF properties of the units do not change systematically.

\section{Model Robustness}

The exact pattern of generalization of the model depended on the parameter settings of the model. The spacing of the spatial inputs during the standard training procedure, as well as the spacing of RFs and the predefined tuning widths in the input and output layer, influenced the model's generalization pattern. Our main goal was to test if such a model could produce asymmetric generalization without the explicit implementation of a forward model for predicting the hand position. Therefore, we varied the following parameters in the respective ranges to probe the robustness of the observed asymmetric generalization: output unit tuning width, [ $\left.\begin{array}{lll}4 & 5 & 6\end{array}\right]$; PD input/ output unit spacing, [ll 24 4]; spatial input spacing, [5 10]; spatial input minimum radius, [l 124$]$; spatial input maximum radius, [12 14]; spatial input spacing, [0.5 1]. Additionally, we varied the network architecture between a pure feedforward implementation and a feedback implementation (Brozovic et al. 2007). The combination of tested variations led to 18 different implementations of the feedback model and 1 imple- mentation of the feedforward model. The strength of the skewness varied with the different parameter combinations. The average skewness across the 18 parameter combinations in the feedback model was $2.01 \pm 0.24$. In 12 out of the 18 individual combinations, the skewness was significantly larger than 0 (i.e., the confidence intervals for the skewness did not overlap with 0 ), while the remaining 6 cases showed a positive trend. Also, the general trends of neuronal changes described in Fig. 5 did not depend on the exact parameter settings. Given the large parameter space (resulting in several thousand possible combinations), and the fact that our conclusions did not depend on an exact fit of the model data to the behavioral results, we did not systematically test the full parameter space, but instead tested only the random subsamples of this parameter space. The results suggest that the positive skewness is a robust finding, since the feedback model in its individual implementations always mimicked the finding in the human psychophysical data, even though the output of the individual implementations never exactly matched the human data.

\section{DISCUSSION}

In this study, we investigated reaching adaptation to target jump. We used a task with unperturbed arm reaches and direct view of the arm to avoid online sensory prediction error about egocentric hand position, but induced consistent local target errors at the end of the movement. The target error in this task was introduced by a target jump that was rendered invisible because of a simultaneous, spatially independent saccade. We found that subjects reliably adapted to the target jump in our paradigm. Adaptation was incomplete, similar to results reported in various previous adaptation paradigms (Krakauer et al. 2000; Martin et al. 1996; Rabe et al. 2009; Schomburg et al. 2014). The adaptation generalized to untrained targets over a finite range, with an asymmetric (skewed) generalization curve. With a neural network model, which was trained to minimize target errors and did not impose asymmetric constraints on the network output, we were able to simulate the observed adaptation patterns of our experiment. The model required neural tuning changes in visuomotor structures to mimic the asymmetric adaptation to target jump observed in the subjects. Based on the combined psychophysical and modeling results, we hypothesize that target jump adaptation might affect spatial response profiles in cortical sensorimotor structures.

\section{Adaptation to Target Jump}

Perturbing sensory feedback during reaching movements reliably leads to sensorimotor adaptation (Kitazawa et al. 1995; Krakauer et al. 2000; Martin et al. 1996; Paz et al. 2003; Redding and Wallace 2006; Shadmehr and Mussa-Ivaldi 1994; Thoroughman and Shadmehr 1999; Vetter et al. 1999). In such experiments, adaptation is typically driven by a combination of sensory prediction error and target error, although prediction error alone can drive adaptation (Mazzoni and Krakauer 2006; Tseng et al. 2007). Target jump perturbation induces only target error, but no sensory feedback error about egocentric hand position (Laurent et al. 2011). Target errors alone evoke adaptation only under specific conditions. For example, target jump paradigms have been used to intentionally induce online corrections of the reach without adaptation (Desmurget et al. 
1999, 2001; Diedrichsen et al. 2005; Goodale et al. 1986; Pelisson et al. 1986). The exact settings of the parameters, such as visibility of hand and target at certain time points, are crucial for determining whether a target jump experiment will not cause adaptation (Desmurget et al. 1999, 2001; Goodale et al. 1986) or will cause adaptation (Cameron et al. 2010, 2011; Laurent et al. 2011, 2012; Magescas et al. 2009; Magescas and Prablanc 2006).

For target errors to cause adaptation, the target offset must be introduced incrementally or rendered invisible by simultaneous saccades (Cameron et al. 2011; Laurent et al. 2012, 2011; Magescas et al. 2009; Magescas and Prablanc 2006). Our experiment falls into the last category and goes beyond the previous studies in that it analyzes and simulates spatial postadaptation generalization to put target jump adaptation into a neuronal context. From the fact that subjects in this paradigm correct for their reach errors, it seems that subjects rather trust a visual stimulus to be stable than they trust the precision of their own motor system.

\section{Generalization in Target Jump Adaptation}

Patterns of generalization in adaptation help to constrain possible underlying mechanisms of adaptation (Donchin et al. 2003; Imamizu et al. 1995; Krakauer et al. 2000; Magescas et al. 2009; Mattar and Ostry 2010, 2007; Pine et al. 1996; Tanaka et al. 2009; Thoroughman and Taylor 2005). Our cross-axis target jump, combined with the arrangement of probe targets along a circle, allowed a close comparison to previous studies using single-target visuomotor rotation. Three features characterized the generalization of target jump adaptation in our data.

First, our target jump adaptation was local. Adaptation effects decayed with distance from the adapted target, as was the case in previous visuomotor rotation experiments (Izawa et al. 2012; Izawa and Shadmehr 2011; Krakauer et al. 2000; Pine et al. 1996). Similar to what is discussed for visuomotor rotation, this suggests that, during target jump adaptation, the units, which are affected by the adaptation, are directionally tuned, and the tuning width of these neurons affects the width of the generalization curve (Thoroughman and Shadmehr 2000). At a more abstract level, this means that adaptation in our target jump task is likely achieved by a local distortion of the visuomotor mapping rather than an off-setting of the origin of any particular spatial frame of reference.

Second, our target jump adaptation induced a translational generalization pattern. Due to the task design, the local crossaxis jump at a single target location did not put any constraints on how the sensorimotor system should interpret the jump; it is compatible with either a rotational offset or a translational offset, since the displaced target practically falls onto the same circumference as the original target. The fact that the sensorimotor system in this case generalizes in a translational direction suggests that adaptations to target error are not carried by a part of the sensorimotor system in which direction and extend of the required movement are represented separately, but rather jointly. This, for example, is the case at a level where reach motor goals are represented in visuospatial coordinates, like the posterior parietal cortex of monkeys (Batista et al. 1999; Buneo et al. 2002). In contrast, adaptation in visuomotor rotation tasks has been shown to affect direction and extend of the reach separately, when the two parameters were perturbed individually (Krakauer et al. 2000). This independent adaptation of reach direction and reach gain was interpreted such that the reach-related neuronal representation, which putatively changed during adaptation, treats these parameters separately. In other words, even though the spatial extent of the generalization is similar between our target jump data and previous visuomotor rotation experiments, the underlying mechanisms might still be distinct.

The first two features of our generalization pattern, being local and translational, are in seeming contrast to one previous study looking at generalization in target jump adaptation (Magescas et al. 2009). In this previous study, subjects adapted to an in-axis target jump. Generalization was observed with similar strength over a large spatial range (approximately \pm 15 $\mathrm{cm})$ in a rectangular array of probe targets. The differences between these and our results could be explained with the results of a recent study (Taylor and Ivry 2013) showing strong influences of the array of probe targets onto the generalization pattern. They found that adaptation to a visuomotor rotation induces local generalization with a circular arrangement of probe targets and broad, translational generalization with a rectangular arrangement of probe targets. In contrast, a translational visuomotor offset with a circular arrangement of probe targets induced local generalization with a bias for a translational generalization pattern, as is the case in our data. In conclusion, if the task constraints allow flexible interpretation, then the context defines how the experienced error is interpreted and defines the resulting adjustments in the sensorimotor system. A rectangular context seems to favor an interpretation that there is a misalignment between the visual and proprioceptive position of the hand, which require a global realignment. This is the case in classic experiments with a translational offset (Ghahramani et al. 1996). A circular context seems to favor an interpretation that there is an offset in the calculation of the motor command required to reach the target, which requires a local correction.

As a third feature, generalization in our target jump data was skewed. In the following two sections we will discuss this asymmetry in the light of two related questions: 1 ) what type of error signal drives learning; and 2) what implications could the result have for underlying neural tuning changes?

\section{Asymmetry in the Generalization and Prediction Error Learning}

Generalization in our experiment showed a spatial decay that was asymmetric in the sense of a skewed generalization curve. This was different from a symmetric, but shifted generalization curve, as seen in a previous study (Gonzalez Castro et al. 2011). The shift in the generalization curve suggested that adaptation works on the experienced rather than the planned motion direction. Asymmetric generalization, as we see it in our experiment, was observed before in visuomotor rotation in healthy subjects, but was absent in cerebellar patients (Izawa et al. 2012). Since, furthermore, the results of Izawa and colleagues (2012) suggest that cerebellar patients are unable to correct for sensory prediction errors, the authors proposed that an asymmetric generalization pattern might be a signature of a system that learns to associate motor commands with sensory 
consequences, i.e., a system that learns by minimizing prediction errors via forward model adaptation.

We consider it very unlikely that, in our target jump, experiment adaptation was driven by errors in predicting hand position, at least not for egocentric hand position. First, adaptation of a forward model based on prediction error learning is typically assumed to take place in cases in which adaptation also induces a shift in perceived hand position (Izawa et al. 2012; Synofzik et al. 2008; but see also Cressman and Henriques 2010). Previous work showed that reach adaptation to target jump does not induce recalibration of the felt hand position (Laurent et al. 2011). Second, subjects in our case had veridical unperturbed feedback about their hand, which makes prediction errors of egocentric hand position implausible. If asymmetric generalization is indeed a signature of prediction error learning, then the target error at the end of the trial would have to be interpreted as sensory prediction error of allocentric hand position, which is possible. In this view, the relevant forward model adapting in our target jump experiment would not predict the expected hand position relative to the body, but instead relative to the target.

As an alternative, asymmetric generalization might not be specific to adaptation based on prediction errors. We tested this hypothesis with our network simulations. Previously, a model simulated the skewed generalization in reach adaptation, using as the error signal the difference between the actual reach direction and the sensory reach direction predicted from the motor command, i.e., a sensory prediction error (Izawa and Shadmehr 2011). We showed here that a model trained on target errors could also produce skewed generalization. In our standard implementation of the network model, the target error at the output stage (motor layer) was back-propagated to sequentially adapt all network weights. Different to Izawa and colleagues, our model had no explicit representation of a sensory prediction of hand position and no implementation of a prediction error-based learning algorithm. Therefore, based on the network model, we conclude that asymmetric generalization is not an indication of prediction error learning.

On the other hand, our model failed to produce positive skewed generalization, as seen in the subjects, when tuning changes in the hidden layer were prevented (see also following section). To achieve this, updating of all recurrent connections, including the back-projections from the motor to the hidden layer, had to be prohibited. If one, in an abstracted way, would consider the bidirectional coupling between hidden and motor layer an equivalent of the idea of a paired inverse and forward model, then the freezing of the feedback projections would correspond to a prevention of forward model learning. In this sense, our network simulations would still be consistent with the idea that asymmetric generalization is an indication of forward model learning. Yet, in this case, forward model learning would not be a consequence of prediction error minimization, but a by-product of target error minimization in a recurrent (bidirectional) sensorimotor association network.

\section{Changes in Visuomotor Tuning During Target Jump Adaptation: A Model-Based Prediction}

Skewed generalization in our model required changes to the neural tuning in the hidden layer. Most psychophysical and theoretical approaches to sensorimotor adaptation argue at a relatively abstract level, e.g., optimal feedback control, without trying to link to the underlying neural implementations. Our neuronal network model is an attempt to make testable predictions about changes in neuronal coding during target jump adaptation. The model produced skewed generalization using a wide range of different parameter sets, but only when tuning changes in the hidden layer were allowed. Our model suggests that asymmetric generalization requires changes in spatial selectivity of neurons in such visuomotor structures.

We speculate that the observed tuning changes in the model correspond to an adaptation of visuospatial motor goals. The tuning properties of the hidden layer units of the original version of our model (Brozovic et al. 2007) closely resembled neural tuning in parietal and premotor cortices of monkeys in an explicitly instructed visuomotor remapping task, namely an anti-reach task (Gail et al. 2009; Gail and Andersen 2006). We predict that, during implicit (noninstructed and mostly unnoticed) adaptation to target jump, the spatial selectivity profiles ("tuning") of neurons in the same frontoparietal areas change. According to the simulation results, units in the sensorimotor areas (hidden layer) with RF positions at the updated location of the target (position after the jump) should increase their firing and their RF size, whereas neurons with RF positions in the opposite direction of the jump should decrease their firing and RF size. As a consequence, the neurons also shift their RF (computed relative to the original target location) opposite to the target jump. On a conceptual level, these neural changes correspond to the idea that subjects implicitly change their target-associated motor goal toward the direction of the target jump.

Only very few studies actually investigated changes due to reach adaptation empirically at a neuronal level (Arce et al. 2010a, 2010b; Mandelblat-Cerf et al. 2009; Paz et al. 2003). Our model predictions are reminiscent of recent findings in the primary motor cortex during force-field adaptation (Mandelblat-Cerf et al. 2009). Our putative implicit change of the motor plan during target jump adaptation seems similar to what conceptually happens during force-field adaptation. In this task, similar to ours, subjects saw a target but virtually aim into a direction that counters the anticipated force field to achieve a movement toward the target. The authors measured neuronal activity in M1 of rhesus monkeys, while the animals adapted to the force field. They found that neurons with a preferred direction (PD) countering the force-field increase their firing rate. Neurons with PDs in direction of the force-field decrease their firing rate. This means, if computed relative to target direction, both neuron types shift their PD in the direction of the force field, corresponding to a shift of the virtual target against the force field.

\section{Summary}

Our target jump paradigm induced reach adaptation effects in response to only target errors. Adaptation effects generalized asymmetrically and stronger in the direction of the target jump. Simulation of this asymmetry in a recurrent neuronal network model is independent of prediction error learning, but contingent on tuning property changes in the hidden layer, suggesting adaptation based on neural tuning changes at early levels of visuospatial reach goal representations. 


\section{ACKNOWLEDGMENTS}

We thank Mark Kaltenhäuser for help in data collection, and Pablo Martinez Vazquez and Ingmar Schwarz for support in data analysis.

\section{GRANTS}

This work was supported by the Federal Ministry for Education and Research (BMBF, Germany) Grants 01GQ0433, 01GQ0814, and 01GQ1005C; the Deutsche Forschungsgemeinschaft Collaborative Research Centre 889; and the State of Lower Saxony Grant VW ZN2563.

\section{DISCLOSURES}

No conflicts of interest, financial or otherwise, are declared by the author(s).

\section{AUTHOR CONTRIBUTIONS}

Author contributions: S.W. and A.G. conception and design of research; S.W. and B.T. performed experiments; S.W., S.K., and B.T. analyzed data; S.W., S.K., B.T., O.D., and A.G. interpreted results of experiments; S.W. prepared figures; S.W. drafted manuscript; S.W., S.K., B.T., O.D., and A.G. edited and revised manuscript; S.W., S.K., B.T., O.D., and A.G. approved final version of manuscript.

\section{REFERENCES}

Arce F, Novick I, Mandelblat-Cerf Y, Israel Z, Ghez C, Vaadia E. Combined adaptiveness of specific motor cortical ensembles underlies learning. J Neurosci 30: 5415-5425, 2010a.

Arce F, Novick I, Mandelblat-Cerf Y, Vaadia E. Neuronal correlates of memory formation in motor cortex after adaptation to force field. $J$ Neurosci 30: 9189-9198, 2010b.

Azzalini A, Capitanio A. Distributions generated by perturbation of symmetry with emphasis on a multivariate skew t-distribution. J R Stat Soc Series B Stat Methodol 65: 367-389, 2003.

Batista AP, Buneo CA, Snyder LH, Andersen RA. Reach plans in eyecentered coordinates. Science 285: 257-260, 1999.

Bedford FL. Constraints on learning new mappings between perceptual dimensions. J Exp Psychol 15: 232-248, 1989.

Bekkering H, Abrams RA, Pratt J. Transfer of saccadic adaptation to the manual motor system. Hum Mov Sci 14: 155-164, 1995.

Bock O. Adaptation of aimed arm movements to sensorimotor discordance: evidence for direction-independent gain control. Behav Brain Res 51: 41-50, 1992.

Brozovic M, Gail A, Andersen RA. Gain mechanisms for contextually guided visuomotor transformations. J Neurosci 27: 10588-10596, 2007.

Buneo CA, Jarvis MR, Batista AP, Andersen RA. Direct visuomotor transformations for reaching. Nature 416: 632-636, 2002.

Cameron BD, Franks IM, Inglis JT, Chua R. Reach adaptation to explicit vs. implicit target error. Exp Brain Res 203: 367-380, 2010.

Cameron BD, Franks IM, Timothy IJ, Chua R. Reach adaptation to online target error. Exp Brain Res 209: 171-180, 2011.

Cressman EK, Henriques DY. Reach adaptation and proprioceptive recalibration following exposure to misaligned sensory input. J Neurophysiol 103: 1888-1895, 2010.

De Graaf JB, Pelisson D, Prablanc C, Goffart L. Modifications in end positions of arm movements following short-term saccadic adaptation. Neuroreport 6: 1733-1736, 1995.

Desmurget M, Epstein CM, Turner RS, Prablanc C, Alexander GE, Grafton ST. Role of the posterior parietal cortex in updating reaching movements to a visual target. Nat Neurosci 2: 563-567, 1999.

Desmurget M, Grea H, Grethe JS, Prablanc C, Alexander GE, Grafton ST. Functional anatomy of nonvisual feedback loops during reaching: a positron emission tomography study. J Neurosci 21: 2919-2928, 2001.

Diedrichsen J, Hashambhoy Y, Rane T, Shadmehr R. Neural correlates of reach errors. J Neurosci 25: 9919-9931, 2005.

Donchin O, Francis JT, Shadmehr R. Quantifying generalization from trial-by-trial behavior of adaptive systems that learn with basis functions: Theory and experiments in human motor control. J Neurosci 23: 9032-9045, 2003.

Fisher NI. Statistical Analysis of Circular Data (1st Ed.). Cambridge, MA: Cambridge University Press 1993.
Gail A, Andersen RA. Neural dynamics in monkey parietal reach region reflect context-specific sensorimotor transformations. J Neurosci 26: 93769384, 2006.

Gail A, Klaes C, Westendorff S. Implementation of spatial transformation rules for goal-directed reaching via gain modulation in monkey parietal and premotor cortex. J Neurosci 29: 9490-9499, 2009.

Georgopoulos AP, Schwartz AB, Kettner RE. Neuronal population coding of movement direction. Science 233: 1416-1419, 1986.

Ghahramani Z, Wolpert DM, Jordan MI. Generalization to local remappings of the visuomotor coordinate transformation. J Neurosci 16: 70857096, 1996.

Gonzalez Castro LN, Monsen CB, Smith MA. The binding of learning to action in motor adaptation. PLoS Comput Biol 7: e1002052, 2011.

Goodale MA, Pelisson D, Prablanc C. Large adjustments in visually guided reaching do not depend on vision of the hand or perception of target displacement. Nature 320: 748-750, 1986.

Imamizu H, Uno Y, Kawato M. Internal representations of the motor apparatus: Implications from generalization in visuomotor learning. $J$ Exp Psychol 21: 1174-1198, 1995.

Izawa J, Criscimagna-Hemminger SE, Shadmehr R. Cerebellar contributions to reach adaptation and learning sensory consequences of action. $J$ Neurosci 32: 4230-4239, 2012.

Izawa J, Shadmehr R. Learning from sensory and reward prediction errors during motor adaptation. PLoS Comput Biol 7: e1002012, 2011.

Kawato M. Internal models for motor control and trajectory planning. Curr Opin Neurobiol 9: 718-727, 1999.

Kitazawa S, Kohno T, Uka T. Effects of delayed visual information on the rate and amount of prism adaptation in the human. J Neurosci 15: 7644$7652,1995$.

Krakauer JW, Ghilardi MF, Mentis M, Barnes A, Veytsman M, Eidelberg D, Ghez C. Differential cortical and subcortical activations in learning rotations and gains for reaching: a PET study. J Neurophysiol 91: 924-933, 2004.

Krakauer JW, Pine ZM, Ghilardi MF, Ghez C. Learning of visuomotor transformations for vectorial planning of reaching trajectories. J Neurosci 20: 8916-8924, 2000.

Laurent D, Sillan O, Prablanc C. Generalization properties of a "saccadiclike" hand-reaching adaptation along a single degree of freedom. Exp Brain Res 216: 609-620, 2012.

Laurent D, Sillan O, Prablanc C. Saccadic-like visuomotor adaptation involves little if any perceptual effects. Exp Brain Res 214: 163-174, 2011.

Magescas F, Prablanc C. A joint-centred model accounts for movement curvature and spatial variability. Neurosci Lett 403: 114-118, 2006.

Magescas F, Urquizar C, Prablanc C. Two modes of error processing in reaching. Exp Brain Res 193: 337-350, 2009.

Mandelblat-Cerf Y, Paz R, Vaadia E. Trial-to-trial variability of single cells in motor cortices is dynamically modified during visuomotor adaptation. $J$ Neurosci 29: 15053-15062, 2009.

Martin TA, Keating JG, Goodkin HP, Bastian AJ, Thach WT. Throwing while looking through prisms. II. Specificity and storage of multiple gazethrow calibrations. Brain 119: 1199-1211, 1996.

Mattar AAG, Ostry DJ. Generalization of dynamics learning across changes in movement amplitude. J Neurophysiol 104: 426-438, 2010.

Mattar AAG, Ostry DJ. Modifiability of generalization in dynamics learning. J Neurophysiol 98: 3321-3329, 2007.

Mazzoni P, Krakauer JW. An implicit plan overrides an explicit strategy during visuomotor adaptation. J Neurosci 26: 3642-3645, 2006.

Paz R, Boraud T, Natan C, Bergman H, Vaadia E. Preparatory activity in motor cortex reflects learning of local visuomotor skills. Nat Neurosci 6: 882-890, 2003.

Pelisson D, Prablanc C, Goodale MA, Jeannerod M. Visual control of reaching movements without vision of the limb. II. Evidence of fast unconscious processes correcting the trajectory of the hand to the final position of a double-step stimulus. Exp Brain Res 62: 303-311, 1986.

Pine ZM, Krakauer JW, Gordon J, Ghez C. Learning of scaling factors and reference axes for reaching movements. Neuroreport 7: 2357-2361, 1996.

Pouget A, Snyder LH. Computational approaches to sensorimotor transformations. Nat Neurosci 3, Suppl: 1192-1198, 2000.

Rabe K, Livne O, Gizewski ER, Aurich V, Beck A, Timmann D, Donchin O. Adaptation to visuomotor rotation and force field perturbation is correlated to different brain areas in patients with cerebellar degeneration. $J$ Neurophysiol 101: 1961-1971, 2009.

Redding GM, Wallace B. Generalization of prism adaptation. J Exp Psychol Hum Percept Perform 32: 1006-1022, 2006. 
Schomburg EW, Fernandez-Ruiz A, Mizuseki K, Berenyi A, Anastassiou CA, Koch C, Buzsaki G. Theta phase segregation of input-specific gamma patterns in entorhinal-hippocampal networks. Neuron 84: 470-485, 2014.

Shadmehr R, Krakauer J. A computational neuroanatomy for motor control. Exp Brain Res 185: 359-381, 2008.

Shadmehr R, Mussa-Ivaldi FA. Adaptive representation of dynamics during learning of a motor task. J Neurosci 14: 3208-3224, 1994.

Shadmehr R, Smith MA, Krakauer JW. Error correction, sensory prediction, and adaptation in motor control. Annu Rev Neurosci 33: 89-108, 2010.

Shadmehr R, Wise SP. Computational Neurobiology of Reaching and Pointing: A Foundation for Motor Learning. Cambridge, MA: MIT Press, 2005.

Synofzik M, Lindner A, Their P. The cerebellum updates predictions about the visual consequences of one's behavior. Curr Biol 18: 814-818, 2008.

Tanaka H, Sejnowski TJ, Krakauer JW. Adaptation to visuomotor rotation through interaction between posterior parietal and motor cortical areas. $J$ Neurophysiol 102: 2921-2932, 2009.
Taylor JA, Ivry RB. Context-dependent generalization. Front Hum Neurosci 7: 171,2013

Thoroughman KA, Shadmehr R. Electromyographic correlates of learning an internal model of reaching movements. J Neurosci 19: 8573-8588, 1999.

Thoroughman KA, Shadmehr R. Learning of action through adaptive combination of motor primitives. Nature 407: 742-747, 2000.

Thoroughman KA, Taylor JA. Rapid reshaping of human motor generalization. J Neurosci 25: 8948-8953, 2005.

Tseng YW, Diedrichsen J, Krakauer JW, Shadmehr R, Bastian AJ. Sensory prediction errors drive cerebellum-dependent adaptation of reaching. J Neurophysiol 98: 54-62, 2007.

Vetter P, Goodbody SJ, Wolpert DM. Evidence for an eye-centered spherical representation of the visuomotor map. J Neurophysiol 81: 935-939, 1999

Wise SP, Moody SL, Blomstrom KJ, Mitz AR. Changes in motor cortical activity during visuomotor adaptation. Exp Brain Res 121: 285-299, 1998.

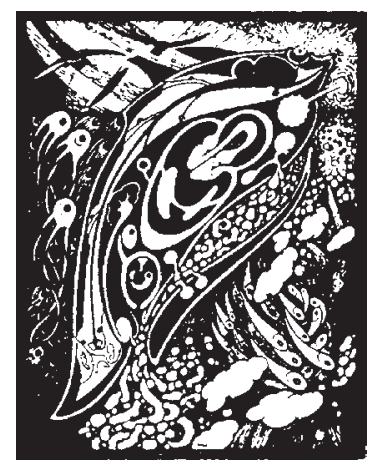




\section{Summary}

In the present thesis, both electrophysiology and psychophysical methods were employed to investigate sensorimotor transformations during reach planning in human and monkey. We were particularly interested in different types of spatial encoding (namely egocentric and allocentric) of the reach parameters during the movement planning.

First, we asked whether presence of task irrelevant visual stimuli during reach planning could yield to an error in the sensorimotor transformation. In a psychophysics study with human subjects, we found that visual contextual information can indeed lead to reach errors. But in short-latency reach movements errors only occurred when they had to be planned in an allocentric frame of reference, not when subjects could conduct egocentric reaches. From the results of this study we predicted that reach planning in the frontoparietal network might have immediate access to the allocentric representation of spatial parameters of the reach (Taghizadeh and Gail, 2014).

Second, in a neurophysiology experiment, we directly tested whether neurons in the reach planning circuit of the frontoparietal network, encode allocentric representations of spatial reach parameters. A rhesus monkey was trained to plan reach movements relative to other objects in an object-centered (that is, allocentric) reference frame. We recorded activity of single neurons from the parietal reach region (PRR) and the dorsal premotor cortex (PMd). We found that a fraction of neurons in the frontoparietal reach network actually encode the location of the reach goal in an object-centered reference frame. To our knowledge, this is the first neuronal evidence that clearly shows single neurons in the posterior parietal cortex encoding spatial parameters of an upcoming reach movement in an allocentric reference frames. This property was characterized by spatial tuning of the neuronal activity to different reach targets on an object, which was independent of the location of the object relative to the monkey.

The common view regarding the allocentric encoding of spatial parameters assigns this role to the areas of the ventral stream such as areas in the temporal lobe (Goodale and Westwood, 2004; Milner and Goodale, 2008). According to this view, the dorsal stream can access allocentric 
information through a long latency period, in the order of seconds. Here we found allocentric representation in sub-regions of the posterior parietal and frontal cortices of the dorsal stream. More importantly, we found that neurons show an allocentric reference frame with short latency of less than 200 milliseconds. This result challenges the traditional view in favor of an alternative model that has recently been more supported by behavioral and imaging studies in human subjects, e.g., (Milner and Goodale, 2008; Burgess, 2006; Thaler and Goodale, 2011). The latter model argues against a distinct separation of allocentric and egocentric encoding in the two visual pathways. It rather suggests that brain areas within the dorsal stream also partly contribute in the formation of an allocentric representation of space.

In subregions of parietal cortex the presence of intermediate egocentric reference frames is a known fact (McGuire and Sabes, 2011; Chang and Snyder, 2010). From a computational perspective, mixed frames of reference are indicative of the main role of these brain areas in transforming information across reference frames (Avillac et al., 2005; Stricanne et al., 1996; Chang and Snyder, 2010; Deneve et al., 2001). At every epoch of the behavioral trial, we see a range of reference frames for the single neurons in the investigated brain areas: covering the full spectrum from egocentric, through intermediate egocentric-allocentric, to allocentric reference frames. We also observed that throughout the course of the behavioral trial, individual neurons varied their reference frames within this spectrum so that they can encode the relevant spatial parameter at different epochs. At the beginning of each behavioral trial, the monkey was provided with the object and the cue (for details, see Behavioral Task in section 2.2). This is just enough information to encode the allocentric location of the reach goal, and not the final egocentric reach goal. Accordingly, at this stage of the sensorimotor transformation we observed that the distribution of the reference frames of individual neurons, in both areas, was biased towards the allocentric end of the spectrum. At the later stage of the trial, the monkey was provided with complementary information to encode the final reach goal. We then see that neurons varied their reference frame as the time proceeded, so that at the later stage of the sensorimotor transformation (before the movement begins) the distribution of the reference frame of individual neurons was biased towards the egocentric end of the spectrum. We suggest that the gradual transition from allocentric to egocentric encoding in a short time window of about 200 milliseconds implies that the transformation between the two types of encoding, at least partly happens within the investigated reach planning circuit. The presence of intermediate 
allocentric-egocentric reference frames at every epoch of the trial also provides additional support for this hypothesis since previous modeling studies (Deneve et al., 2001; Avillac et al., 2005) showed that intermediate reference frames exist in the network of neurons which perform reference frame transformation. Our data adds a new dimension to the common view about the role of the parietal cortex in spatial transformations, and suggests that posterior parietal cortex can also be involved in transformation between egocentric and allocentric representation.

In a complementary electrophysiology experiment, we investigated how the spatial selectivity of neurons is influenced by spatial features of the object itself, not just its location. We studied the spatial tuning of the neurons to different targets on the object, when the reach goals were localized on a long object as compared to when the reach goals were localized on a shorter version of the same object. A subset of neurons scaled the width of their tuning profile to the size of the object. This property was characterized by a spatial tuning of the neuronal activity to different reach targets on the object, which was independent of the object size and location. Our preliminary results suggest that different neurons scale their tuning width to different degrees: in both areas we see a range of fully-scaled object-centered to a non-scaled egocentric spatial tuning. Hence, spatial selectivity of the neurons not only adapts to the cognitive task demands within a few hundred milliseconds within a trial (as was evident in the first experiment), but also changes on a longer time scale from trial to trial in order to encode the task relevant parameters. In general, the data provides novel evidence that the spatial selectivity of neurons in PRR and PMd, depends on the spatial task contexts rather than being a fixed and predefined property as in sensory areas.

Altogether, our electrophysiology experiments show that individual neurons in the reach circuit of the frontoparietal network dynamically vary their spatial selectivity to subserve what the behavior demands. The changes happen in different time scales: from a few hundred milliseconds (which happened within one behavioral trial) to a few second (which happened across the trials).

In situations where environmental factors cause an error in the sensorimotor transformation, the sensory to motor mapping needs to be adjusted in order to compensate for the error. Depending on the type of the external perturbation adjustments could happen in different time scales: short time scale, e.g., when reaching to an object and trying to avoid an obstacle which is 
unpredictably moving in the environment; long time scale, e.g., learning to throw a ball in a basket while wearing shifting prism glasses. Flexibility of the neuronal encoding is beneficial for flexible sensorimotor transformations which is robust to dynamics and noisiness of the ambient environment, and manipulations of the sensory inputs.

Our third investigation addressed motor adaptation and provided complementary behavioral evidence in support of such flexibility in sensorimotor transformations. By displacing the reach target in one location of the fronto-parallel space, subjects faced a situation in which they failed to precisely localize the reach goal only for one target direction but not for all others due to a subliminal manipulation of the reach target. Subjects learned to reach to the perturbed target; the learning procedure required adjustment of the sensorimotor transformation to overcome the perturbation. Experimental result together with neural network simulation suggested that throughout the learning procedure, the motor goal representation was gradually updated in the sensorimotor network. Result of this study showed that a local perturbation led to a local adjustment of the sensorimotor transformation around the perturbed target location rather than inducing a global offset in the center of the frame of reference which was used to encode the target location.

In summary, this thesis presents work that provided behavioral and neuronal evidence emphasizing dynamic and flexible sensorimotor transformations within the reach planning circuit of the frontoparietal network. It revealed a novel and abstract representation of the spatial reach goal in object-centered reference frame, within both areas PRR and PMd. It further shows how neurons flexibly vary their selectivity depending on the spatial task demands, which is a crucial feature for flexible sensorimotor transformations. 


\section{Bibliography}

1. Andersen RA, Andersen KN, Hwang EJ, Hauschild M (2014) Optic ataxia: from Balint's syndrome to the parietal reach region. Neuron 81: 967-983.

2. Andersen RA, Essick GK, Siegel RM (1985) Encoding of spatial location by posterior parietal neurons. Science 230: 456-458.

3. Avillac M, Deneve S, Olivier E, Pouget A, Duhamel JR (2005) Reference frames for representing visual and tactile locations in parietal cortex. Nat Neurosci 8: 941-949.

4. Batista AP, Buneo CA, Snyder LH, Andersen RA (1999) Reach plans in eye-centered coordinates. Science 285: 257-260.

5. Batista AP, Santhanam G, Yu BM, Ryu SI, Afshar A, Shenoy KV (2007) Reference frames for reach planning in macaque dorsal premotor cortex. J Neurophysiol 98: 966-983.

6. Battaglia-Mayer A, Caminiti R, Lacquaniti F, Zago M (2003) Multiple levels of representation of reaching in the parieto-frontal network. Cereb Cortex 13: 1009-1022.

7. Bernier PM, Grafton ST (2010) Human posterior parietal cortex flexibly determines reference frames for reaching based on sensory context. Neuron 68: 776-788.

8. Beurze SM, Toni I, Pisella L, Medendorp WP (2010) Reference frames for reach planning in human parietofrontal cortex. J Neurophysiol 104: 1736-1745.

9. Biran I, Coslett HB (2003) Visual agnosia. Curr Neurol Neurosci Rep 3: 508-512.

10. Boussaoud D, di PG, Wise SP (1995) Frontal lobe mechanisms subserving vision-foraction versus vision-for-perception. Behav Brain Res 72: 1-15.

11. Boussaoud D, Wise SP (1993) Primate frontal cortex: effects of stimulus and movement. Exp Brain Res 95: 28-40.

12. Buneo CA, Andersen RA (2006) The posterior parietal cortex: sensorimotor interface for the planning and online control of visually guided movements. Neuropsychologia 44: 2594-2606.

13. Buneo CA, Jarvis MR, Batista AP, Andersen RA (2002) Direct visuomotor transformations for reaching. Nature 416: 632-636.

14. Burgess N (2006) Spatial memory: how egocentric and allocentric combine. Trends Cogn Sci 10: 551-557. 
15. Caminiti R, Johnson PB, Galli C, Ferraina S, Burnod Y (1991) Making arm movements within different parts of space: the premotor and motor cortical representation of a coordinate system for reaching to visual targets. J Neurosci 11: 1182-1197.

16. Chafee MV, Averbeck BB, Crowe DA (2007) Representing spatial relationships in posterior parietal cortex: single neurons code object-referenced position. Cereb Cortex 17: 2914-2932.

17. Chang SW, Snyder LH (2010) Idiosyncratic and systematic aspects of spatial representations in the macaque parietal cortex. Proc Natl Acad Sci U S A 107: 7951-7956.

18. Chen Y, Monaco S, Byrne P, Yan X, Henriques DY, Crawford JD (2014) Allocentric versus Egocentric Representation of Remembered Reach Targets in Human Cortex. J Neurosci 34: 12515-12526.

19. Cisek P, Kalaska JF (2010) Neural mechanisms for interacting with a world full of action choices. Annu Rev Neurosci 33: 269-298.

20. Cohen YE, Andersen RA (2002) A common reference frame for movement plans in the posterior parietal cortex. Nat Rev Neurosci 3: 553-562.

21. Colby CL (1998) Action-oriented spatial reference frames in cortex. Neuron 20: 15-24.

22. Colby CL, Gattass R, Olson CR, Gross CG (1988) Topographical organization of cortical afferents to extrastriate visual area PO in the macaque: a dual tracer study. J Comp Neurol 269: 392-413.

23. Committeri G, Galati G, Paradis AL, Pizzamiglio L, Berthoz A, LeBihan D (2004) Reference frames for spatial cognition: different brain areas are involved in viewer-, object, and landmark-centered judgments about object location. J Cogn Neurosci 16: 1517-1535.

24. Coslett HB, Lie G (2008) Simultanagnosia: when a rose is not red. J Cogn Neurosci 20: 3648.

25. Crawford JD, Medendorp WP, Marotta JJ (2004) Spatial transformations for eye-hand coordination. J Neurophysiol 92: 10-19.

26. Deneve S, Latham PE, Pouget A (2001) Efficient computation and cue integration with noisy population codes. Nat Neurosci 4: 826-831.

27. Dum RP, Strick PL (2005) Frontal lobe inputs to the digit representations of the motor areas on the lateral surface of the hemisphere. J Neurosci 25: 1375-1386.

28. Essen DC, Zeki SM (1978) The topographic organization of rhesus monkey prestriate cortex. J Physiol 277: 193-226.

29. Gail A, Andersen RA (2006) Neural dynamics in monkey parietal reach region reflect context-specific sensorimotor transformations. J Neurosci 26: 9376-9384. 
30. Galati G, Lobel E, Vallar G, Berthoz A, Pizzamiglio L, Le Bihan D (2000) The neural basis of egocentric and allocentric coding of space in humans: a functional magnetic resonance study. Exp Brain Res 133: 156-164.

31. Galati G, Pelle G, Berthoz A, Committeri G (2010) Multiple reference frames used by the human brain for spatial perception and memory. Exp Brain Res 206: 109-120.

32. Gamberini M, Passarelli L, Fattori P, Zucchelli M, Bakola S, Luppino G, Galletti C (2009) Cortical connections of the visuomotor parietooccipital area V6Ad of the macaque monkey. J Comp Neurol 513: 622-642.

33. Goodale MA, Milner AD (1992) Separate visual pathways for perception and action. Trends Neurosci 15: 20-25.

34. Goodale MA, Westwood DA (2004) An evolving view of duplex vision: separate but interacting cortical pathways for perception and action. Curr Opin Neurobiol 14: 203-211.

35. Gross RG, Grossman M (2008) Update on apraxia. Curr Neurol Neurosci Rep 8: 490-496.

36. Hwang EJ, Andersen RA (2012) Spiking and LFP activity in PRR during symbolically instructed reaches. J Neurophysiol 107: 836-849.

37. Iriki A, Tanaka M, Iwamura Y (1996) Coding of modified body schema during tool use by macaque postcentral neurones. Neuroreport 7: 2325-2330.

38. Johnson PB, Ferraina S, Bianchi L, Caminiti R (1996) Cortical networks for visual reaching: physiological and anatomical organization of frontal and parietal lobe arm regions. Cereb Cortex 6: 102-119.

39. Kakei S, Hoffman DS, Strick PL (1999) Muscle and movement representations in the primary motor cortex. Science 285: 2136-2139.

40. Kalaska JF, Crammond DJ (1995) Deciding not to GO: neuronal correlates of response selection in a GO/NOGO task in primate premotor and parietal cortex. Cereb Cortex 5: 410-428.

41. Kalaska JF, Scott SH, Cisek P, Sergio LE (1997) Cortical control of reaching movements. Curr Opin Neurobiol 7: 849-859.

42. Klaes C, Westendorff S, Chakrabarti S, Gail A (2011) Choosing goals, not rules: deciding among rule-based action plans. Neuron 70: 536-548.

43. Kravitz DJ, Saleem KS, Baker CI, Mishkin M (2011) A new neural framework for visuospatial processing. Nat Rev Neurosci 12: 217-230.

44. Kurata K (1991) Corticocortical inputs to the dorsal and ventral aspects of the premotor cortex of macaque monkeys. Neurosci Res 12: 263-280. 
45. Lacquaniti F, Caminiti R (1998) Visuo-motor transformations for arm reaching. Eur J Neurosci 10: 195-203.

46. Marconi B, Genovesio A, Battaglia-Mayer A, Ferraina S, Squatrito S, Molinari M, Lacquaniti F, Caminiti R (2001) Eye-hand coordination during reaching. I. Anatomical relationships between parietal and frontal cortex. Cereb Cortex 11: 513-527.

47. Martin TA, Keating JG, Goodkin HP, Bastian AJ, Thach WT (1996) Throwing while looking through prisms. II. Specificity and storage of multiple gaze-throw calibrations. Brain 119 ( Pt 4): 1199-1211.

48. Matelli M, Govoni P, Galletti C, Kutz DF, Luppino G (1998) Superior area 6 afferents from the superior parietal lobule in the macaque monkey. J Comp Neurol 402: 327-352.

49. McGuire LM, Sabes PN (2009) Sensory transformations and the use of multiple reference frames for reach planning. Nat Neurosci 12: 1056-1061.

50. McGuire LM, Sabes PN (2011) Heterogeneous representations in the superior parietal lobule are common across reaches to visual and proprioceptive targets. J Neurosci 31: 6661-6673.

51. Medina J, McCloskey M, Coslett HB, Rapp B (2014) Somatotopic representation of location: Evidence from the Simon effect. J Exp Psychol Hum Percept Perform 40: 21312142.

52. Milner AD, Goodale MA (2008) Two visual systems re-viewed. Neuropsychologia 46: 774-785.

53. Milner AD, Perrett DI, Johnston RS, Benson PJ, Jordan TR, Heeley DW, Bettucci D, Mortara F, Mutani R, Terazzi E, . (1991) Perception and action in 'visual form agnosia'. Brain 114 ( Pt 1B): 405-428.

54. Mishkin M, Ungerleider LG (1982) Contribution of striate inputs to the visuospatial functions of parieto-preoccipital cortex in monkeys. Behav Brain Res 6: 57-77.

55. Rushworth MF, Paus T, Sipila PK (2001) Attention systems and the organization of the human parietal cortex. J Neurosci 21: 5262-5271.

56. Scott SH, Kalaska JF (1995) Changes in motor cortex activity during reaching movements with similar hand paths but different arm postures. J Neurophysiol 73: 2563-2567.

57. Scott SH, Kalaska JF (1997) Reaching movements with similar hand paths but different arm orientations. I. Activity of individual cells in motor cortex. J Neurophysiol 77: 826852.

58. Snyder LH, Batista AP, Andersen RA (1997) Coding of intention in the posterior parietal cortex. Nature 386: 167-170. 
59. Snyder LH, Batista AP, Andersen RA (2000) Intention-related activity in the posterior parietal cortex: a review. Vision Res 40: 1433-1441.

60. Soechting JF, Flanders M (1989) Sensorimotor representations for pointing to targets in three-dimensional space. J Neurophysiol 62: 582-594.

61. Stricanne B, Andersen RA, Mazzoni P (1996) Eye-centered, head-centered, and intermediate coding of remembered sound locations in area LIP. J Neurophysiol 76: 20712076.

62. Taghizadeh B, Gail A (2014) Spatial task context makes short-latency reaches prone to induced Roelofs illusion. Front Hum Neurosci 8: 673.

63. Thaler L, Goodale MA (2011) Neural substrates of visual spatial coding and visual feedback control for hand movements in allocentric and target-directed tasks. Front Hum Neurosci 5: 92.

64. Vallar G, Lobel E, Galati G, Berthoz A, Pizzamiglio L, Le BD (1999) A fronto-parietal system for computing the egocentric spatial frame of reference in humans. Exp Brain Res 124: 281-286.

65. Weinrich M, Wise SP (1982) The premotor cortex of the monkey. J Neurosci 2: 13291345.

66. Westendorff S, Klaes C, Gail A (2010) The cortical timeline for deciding on reach motor goals. J Neurosci 30: 5426-5436.

67. Westwood DA, Goodale MA (2011) Converging evidence for diverging pathways: neuropsychology and psychophysics tell the same story. Vision Res 51: 804-811.

68. Wise SP, Boussaoud D, Johnson PB, Caminiti R (1997) Premotor and parietal cortex: corticocortical connectivity and combinatorial computations. Annu Rev Neurosci 20: 2542.

69. Zaehle T, Jordan K, Wustenberg T, Baudewig J, Dechent P, Mast FW (2007) The neural basis of the egocentric and allocentric spatial frame of reference. Brain Res 1137: 92-103. 


\section{Curriculum vitae}

BAHAREH TAGHIZADEH

btaghizadeh@dpz.eu

\section{EDUCATION}

PhD student in Neuroscience, 2010-2015

Sensorimotor group of the Bernstein Center for Computational Neuroscience (BCCN) in Goettingen, headed by Prof. Dr. Alexander Gail. GEORG-AUGUST-UNIVERSITÄT GÖTTINGEN.

Research Subject: Sensorimotor transformations in goal directed reach movements.

\section{M.S. in Biomedical Engineering (Bioelectricity)}

Amirkabir University of Technology, Tehran, Iran

2006-2009

Thesis title: Model for Human Path Planning, Using Model Based Predictive Control.

\section{B.S. in Electrical Engineering (Tele-Communication Systems)}

Ferdowsi University of Mashhad, Mashhad, Iran.

2001-2006

Thesis title: Implementation of a Monocular Model-Based 3D Tracking System for Augmented Reality.

\section{Diploma in Mathematics and Physics}

Farzanegan High School, National Organization for Development of Exceptional Talents, Mashhad, Iran.

$1997-2001$ 


\section{JOURNAL PUBLICATION}

Taghizadeh B and Gail A (2014) Spatial task context makes short- latency reaches prone to induced Roelofs illusion. Front. Hum. Neurosci. 8:673. DOI:

0.3389/fnhum.2014.00673.

Westendorff, S, Kuang, S, Taghizadeh, B, Donchin, O, Gail, A, Asymmetric generalization in adaptation to target displacement errors in humans and in a neural network model. J. Neurophysiology 113(7): 2360-2375. DOI: 10.1152/jn.00483.2014

Morel P, Ferrea E, Taghizadeh-Sarshouri B, Cardona Audí JM, Ruff R, Hoffmann K-P, Lewis S, Russold M, Dietl H, Abu-Saleh L, Schroeder D, Krautschneider W, Meiners T, Gail.A (2016) Long-term decoding of movement force and direction with a wireless myoelectric implant.J. Neural Engineering 13(1):016002. DOI: 10.1088/17472560/13/1/016002

\section{PUBLISHED ABSTRACT}

Taghizadeh B., Gail A., “Object-centered representations in monkey parietal reach region and dorsal premotor cortex", 44th Annual meeting of Society for Neuroscience, Washington D.C., USA, 2014.

Taghizadeh B., Gail A., “Object-centered spatial encoding in monkey parietal reach region and dorsal premotor cortex”, 14th FENS forum, Milan, Italy, 2014.

Taghizadeh B., Gail A., “Object-centered representations in monkey parietal reach region and dorsal premotor cortex”, 43rd Annual meeting of Society for Neuroscience, San Diego, USA, 2013.

Taghizadeh B., Gail A., “Object-centered representations in monkey parietal reach region and dorsal premotor cortex”, Bernstein Conference (BC13), Tuebingen, Germany, 2013.

Taghizadeh B., Gail A., “Allocentric planning of immediate reach movement is prone to 
induced Roelofs illusion”, 10th Goettingen meeting of German Neuroscience Society, Goettingen, Germany, 2013.

Taghizadeh B., Gail A., "Object-centered reach planning is subject to the induced Roelofs effect”, 5th Annual Primate Neurobiology meeting, Tuebingen, Germany, 2012.

Taghizadeh B., Gail A., "Induced Roelofs effect in reaching”, Bernstein Conference (BC11), Freiburg, Germany, 2011.

\section{SELECTED COURSES/ WORKSHOPS}

Trans cranial magnetic and electrical stimulation - 2013

EUPRIM-Net Course on General Primate Biology - 2011

NWG course on analysis and models in neurophysiology - 2010 